\title{
The role of male song in spatial behavior and reproductive decisions in the great tit (Parus major)
}

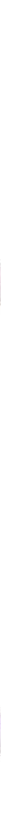

Nina Bircher 


\section{Propositions}

1) The dawn song of male song birds does not necessarily serve as a deterrent to other males (this thesis).

2) Automated long-term tracking allows for insights into responses to animal signals that would otherwise go undetected (this thesis).

3) Philosophy is an important source of inspiration for scientific theory and experiments.

4) Publication of non-significant results is important for scientific progress.

5) Teaching staff should use and promote, whenever possible, open source programs that are available to all students after leaving the University.

6) Treating other animals as commodity is morally wrong.

\section{Propositions belonging to the thesis, entitled:}

The role of male song in spatial behavior and reproductive decisions in the great tit (Parus major)

Nina Bircher

Wageningen, 9 November 2020 

The role of male song in spatial behavior and reproductive decisions in the great tit (Parus major) 


\section{Thesis committee}

\section{Promotors}

Prof. Dr Marc Naguib

Professor of Behavioral Ecology, Wageningen University \& Research

Prof. Dr Kees van Oers

Special Professor of Animal Personality, Wageningen University \& Research / Netherlands Institute of Ecology (NIOO-KNAW)

\section{Other members}

Prof. Dr Frank van Langevelde, Wageningen University \& Research

Dr Katharina Riebel, Leiden University

Dr Robert F. Lachlan, Royal Holloway University of London, UK

Dr Wouter Halfwerk, Vrije Universiteit Amsterdam

This research was conducted under the auspices of the Graduate School Wageningen Institute of Animal Sciences. 


\title{
The role of male song in spatial behavior and reproductive decisions in the great tit (Parus major)
}

\author{
Nina Bircher
}

\section{Thesis}

submitted in fulfilment of the requirements for the degree of doctor at Wageningen University by the authority of the Rector Magnificus,

Prof. Dr A.P.J. Mol,

in the presence of the

Thesis Committee appointed by the Academic Board to be defended in public

on Monday 9 November 2020

at $1.30 \mathrm{pm}$ in the Aula. 
Nina Bircher

The role of male song in spatial behavior and reproductive decisions in the great tit (Parus major), 166 pages.

PhD thesis, Wageningen University, Wageningen, the Netherlands (2020)

With references, with summary in English

ISBN: 978-94-6395-543-0

DOI: https://doi.org/10.18174/531278 


\section{Table of contents}

$\begin{array}{lll}\text { Chapter } 1 & \text { General introduction }\end{array}$

Chapter 2 Extra-territorial forays by great tits are associated with dawn song in 21 unexpected ways

Chapter 3 No evidence for eavesdropping on male singing interactions in the great tit (Parus major)

Chapter 4 Does singing behavior during song contests reflect relative differences between males in the great tit (Parus major)?

Chapter 5 Foraying activity to other territories is associated with pair personality in the great tit (Parus major)

Chapter 6 General discussion

References

Summary

Acknowledgements 163

Curriculum vitae

Wias Training and Education Statement 166 

CHAPTER 1

General introduction 

In many animal species, males have developed elaborate ornaments and displays (Andersson, 1994; Bradbury and Vehrencamp, 1998). The collection of colorful feathers, mighty antlers, and sophisticated dances that at first sight do not aid an individual's fitness, have fascinated and puzzled biologists for a long time. These ornaments and displays may communicate phenotypic or genetic quality and serve as honest signals of male quality because they are costly to produce. Direct costs for the signaler can control cheating if only the fittest individuals can afford to produce the most elaborate ornaments or displays (Grafen, 1990; Zahavi, 1975) or through the behavior of receivers (for example through social aggression) (Guilford and Dawkins, 1995). Many elaborate signals often serve as both "armaments and ornaments" (Berglund et al., 1996; but see for example Leitão and Riebel, 2003), as they play an essential role in the competition between males over access to resources and the attraction of females.

Since Darwin observed in 1871 that "the sweet strains poured forth by the males during the season of love are certainly admired by the females" (Darwin, 1871), bird song has been studied extensively and became, alongside elaborate male plumage displays, a textbook example of a sexually selected ornament (Andersson, 1994; Bradbury and Vehrencamp, 1998; Catchpole and Slater, 2008). Song is a costly and honest signal of male quality (reviewed in Gil and Gahr, 2002) and its general dual function of female attraction and territory or resource defense is supported by a vast amount of correlative and experimental studies (Catchpole and Slater, 2008; McGregor, 1991; Naguib and Riebel, 2006; Searcy and Yasukawa, 1996). More recently, this classic view that sexual dimorphism in song is the result of sexual selection acting on males and female song is a rare exception has been debated. Female song is much more common and elaborate than previously thought, especially in the tropics, and seems to be the ancestral state in many species (Odom et al., 2014; Price, 2015; Riebel et al., 2019, 2005). However, even in temperate species, were song is predominantly a male trait (Morton, 1996), many questions about the role of song remain unanswered, despite decades of research. Since singing occurs in a communication network (McGregor et al., 2000; McGregor and Peake, 2000), it is inherently difficult to know to which audience a bird precisely is singing at a given time as the same signal can be directed at and heard by multiple receivers. Moreover, since many receivers' movement is often cryptic and difficult to follow, some responses of potential audiences may go unobserved (Bircher and Naguib, 2020; Snijders and Naguib, 2017). The reasons why so many bird species show a conspicuous peak of singing activity around dawn ("dawn chorus"), for example, remain elusive (Gil and Llusia, 2020). Two relevant hypotheses for singing at dawn, namely that this time is particularly crucial for territory defense and the attraction of extra-pair 
mates (Gil and Llusia, 2020; Kacelnik and Krebs, 1983; Staicer et al., 1996), would benefit from more detailed information about the actual spatial behavior of females and males at this time. Tracking in the early morning (Amrhein et al., 2004a; Double and Cockburn, 2000) and other times of the day in more species would show whether dawn singing predicts the movements of these potential receivers generally. Moreover, bird songs are often highly interactive (Burt and Vehrencamp, 2005; Naguib, 2005; Todt and Naguib, 2000), but have initially been studied using non-interactive playback experiments, where playback stimuli were broadcast independently of the study subject's response (King, 2015). With the extensive use of interactive playbacks in later studies, where the timing and type of stimulus change according to how the subject responds, it soon became evident that some of the information in male singing is conveyed only through specific patterns in the interaction itself, not by each signal alone (Catchpole and Slater, 2008; Dabelsteen, 1992; Naguib, 2005). In this thesis, I combined automated radio-tracking and audio recording and interactive playbacks to study the role of dawn song and singing patterns in vocal interactions in the spatial behavior and female reproductive decisions, using the great tit (Parus major) as a study system.

\section{Dawn song - attracting females and repelling males?}

The dawn chorus is a daily period of high singing activity in the early morning that occurs in most passerines and some non-passerines during the breeding season. This burst of singing activity starts around nautical twilight for the earliest species and is joined by other species in a more or less defined order until it ends approximately around sunrise (Gil and Llusia, 2020). Several non-exclusive hypotheses have been suggested to explain why so many bird species show a higher singing activity at this particular time of day (Gil and Llusia, 2020; Kacelnik and Krebs, 1983; Staicer et al., 1996). An early peak of singing may be based on circadian hormone cycles and caused by a peak of hormones around dawn that stimulates singing, irrespective of adaptive functions (circadian hormone cycle hypotheses) (Staicer et al., 1996). Alternatively, the time around dawn may be a time window with particularly favorable conditions for singing due to lower predation risk and better or at least more constant sound transmission conditions (low predation risk and better sound transmission hypotheses) (Henwood and Fabrick, 1979; Staicer et al., 1996).

On the other hand, dawn might just be a time of unfavorable conditions for other important activities; for example, it might be inefficient for foraging because of low light levels and temperature (inefficient foraging hypotheses) (Kacelnik, 1979). As conditions overnight are often unpredictable, birds may end up with extra energy in the morning that they store in 
preparation for the night and use it at dawn for singing (unpredictable overnight conditions hypotheses) (McNamara et al., 1987).

\section{Dawn song for territory defense and resolving social relationships}

Dawn may be an especially critical time for territory defense: territory vacancy might be high after the night, as no new males will settle during the night, and mortality overnight might be higher. Thus, "floater" males that do not hold a territory yet should be prospecting and try to settle during this time, whereas established territory holders should be more on guard and invest in signaling territory occupancy and social status. Moreover, social relationships among existing territory holders are dynamic (Burt and Vehrencamp, 2005; Møller, 1990), and communication between them is interrupted by night. Thus, dawn may be in general a time of higher territorial instability and simultaneously poses the first opportunity after a prolonged interruption for information exchange and resettling territory boundaries as well as dominance relationships. Singing very actively to announce presence and status during this particular time may thus pay off for resident males (territory defense and social dynamics hypotheses) (Kacelnik and Krebs, 1983). Multiple studies have indeed provided evidence that dawn song is important for settling boundaries and hierarchies (Burt and Vehrencamp, 2005; Foote et al., 2010). Dawn song is often more complex and elaborate than daytime song (reviewed in Staicer et al. 1996). It can be especially rich on interactions with higher levels of signal matching (Foote et al., 2008a), especially loud or complex (Trillo and Vehrencamp, 2005) or sung at higher rates compared to song during the day (Amrhein et al., 2004b; Liu and Kroodsma, 2007), which indicates that singing more aggressively or elaborately during this time is important.

Moreover, experimentally increasing intruder presences led to males increasing their dawn song activity in some species the day after the manipulation (Amrhein and Erne, 2006; Amrhein and Lerch, 2010; Foote et al., 2011; Liu, 2004). That males continue to sing after the fertile period of their female in some species, might also indicate that dawn song plays a role in territory defense (Amrhein et al., 2008, 2004a; Liu and Kroodsma, 2007). Some studies also reported that dawn song is often produced at territory boundaries, indicating that it is crucial for settling boundary disputes (Bolsinger, 2000; Lein, 2007; Liu and Kroodsma, 2007), although in other species, males sing primarily near the nest or in the center of their territories (Mace, 1986; Møller, 1988; Pärt, 1991). There is some evidence that intruder pressure may be particularly high at dawn, as intrusion rates or male prospecting activity during dawn appear to be higher than during the day (Amrhein et al., 2004a; Dalziell and Cockburn, 2008; Kacelnik and Krebs, 1983). Detailed radio-tracking of males at dawn and other times of the day in more species will 
help get a clearer picture of intrusion rates and sneaking behavior and whether dawn is indeed a time of higher intrusion pressure and territorial instability (Gil and Llusia, 2020).

\section{Dawn song for manipulating female mating behavior}

Another reason why birds sing particularly actively at dawn might be the manipulation of female mating behavior around the time of fertility, i.e., to protect paternity against intruding males when the female is fertile and to ensure receptivity of the female to copulations with the singing male. Female birds' fertile window is generally thought to start some days before the beginning of egg-laying to some days after. Within that window, it has been suggested that female birds are particularly fertile right after laying (thus in many species early in the morning) (Birkhead, 1992; Birkhead and Møller, 1992; Cheng et al., 1983), although the idea that females are particularly fertile right after laying is debated (Birkhead et al., 1996). Therefore, it may pay males to sing more actively and elaborately both during the days of the season on which their mate is fertile and early in the morning (fertility insurance hypotheses) (Mace, 1987). There is some evidence that mating rates are indeed higher early in the morning in the wild (Drachmann et al., 1997). In the European starling (Sturnus vulgaris), a species were females lay eggs not at dawn but in the late morning, male singing and mating peaks indeed in the late morning, and not dawn, on days when females are fertile (Pinxten and Eens, 1998), suggesting that singing is linked to the female's fertility window. In many species, dawn song activity indeed peaks during the fertile days of females (Bruni and Foote, 2014; Cuthill and Macdonald, 1990; Mace, 1987; Møller, 1988; Sexton et al., 2007). However, although male dawn singing activity is often high during this time, males also sing before and after the fertile period of their mate (Forstmeier and Balsby, 2002; Gil et al., 1999b; Naguib et al., 2016) and in some cases, dawn singing is not related to female fertility (Kunc et al., 2005; Moran et al., 2019; Slagsvold, 1996).

However, dawn singing may be crucial for securing copulations with a male's partner and other fertile females, which would explain why males in some species are also singing when their mate is not fertile. Several studies provide evidence that dawn may be an important time for extra-pair copulations: In the superb fairy-wren females make extra-territorial forays just before dawn, presumably to avoid detection by their mates (Double and Cockburn, 2000) and female black-capped chickadees sometimes copulate with extra-pair mates during twilight at dawn (Mennill et al., 2004). In great tits, females that ended up having extra-pair offspring emerged earlier from nest boxes at dawn during the time just before egg-laying (Halfwerk et al., 2011; 
but see Schlicht et al., 2014 for no such relationship in blue tits) and males that were experimentally induced to sleep longer with melatonin were more likely to be cuckolded (Greives et al., 2015). Several studies have provided evidence for an association between individual differences in dawn singing behavior in males and their success in siring extra-pair offspring or avoiding loss of paternity in their own nest (Halfwerk et al., 2011; Kempenaers et al., 2010, 1997; Poesel et al., 2006), although a recent meta-analysis found no association between the considered song traits and occurrence of extra-pair offspring. However, this analysis did not include dawn song only (Garamszegi, 2004). Detailed tracking of the movement of females in more species and for more extended periods is necessary to understand whether they are more likely to foray early in the morning, whether these movements are in any way associated with the resident's male singing behavior and whether they may be linked to extra-pair mating.

\section{Song matching and overlapping - a form of vocal dueling?}

Observing competitive interactions between other individuals can provide an opportunity to assess the fighting ability, motivation, or social status of contestants and adjust one's behavior in response, for example in territory disputes or mate choice (Cheney and Seyfarth, 1990; Freeman, 1987; Johnson and Akerman, 1998; Oliveira et al., 1998; Silk, 1999). Male songbirds often engage in singing interactions with two males singing at the same time in a back and forth fashion (Catchpole and Slater, 2008; Todt and Naguib, 2000). There are multiple singing strategies a male can employ when interacting with a rival, as it can choose both the timing and the type of its signal in response. A male could, for example, produce either a different ("nonmatching") or the same signal type ("matching") as the opponent (Todt, 1981). Similarly, it could either alternate singing with the other individual ("alternating") or overlap the signal of the other in time ("overlapping") (Hultsch and Todt, 1982; Naguib, 2005; Todt and Naguib, 2000). Signal matching and overlapping could occur due to chance alone, but may also be produced intentionally. In songbirds, the way a male responds to another in interactions, i.e., whether and how often it overlaps or matches the respective opponent, has been suggested to provide relevant information about the signaler (reviewed in McGregor and Peake 2000; Naguib 2005; Searcy and Beecher 2009; Naguib and Mennill 2010; Helfer and Osiejuk 2015). The nature of this information is still debated (Helfer and Osiejuk, 2015; Naguib and Mennill, 2010; Searcy and Beecher, 2011, 2009), but a common interpretation is that both matching and overlapping signal aggressive intention or close approach and motivation to escalate an interaction (Helfer and Osiejuk, 2015; Naguib, 2005; Naguib and Mennill, 2010; Searcy and 
Beecher, 2009; Vehrencamp, 2001). Observing asymmetries in the usage of matching and overlapping between interacting males may thus be a way for eavesdropping individuals to gather information about the relationship between others (McGregor, 1993; Valone and Templeton, 2002). This information could only be gained by paying attention to the interaction between males and not listening to a solo singer (Dabelsteen and McGregor, 1996; King, 2015). Males might eavesdrop on such interactions to assess the threat of a potential intruder or rival (Hall et al., 2006; Mennill and Ratcliffe, 2004; Naguib et al., 2004, 1999; Naguib and Todt, 1997; Peake et al., 2002, 2001) and females to assess the quality of (potential) mates (Amy et al., 2008; Garcia-Fernandez et al., 2010; Mennill et al., 2003; Otter et al., 1999). Multiple studies have indeed provided evidence that both males and females respond differently to males that match and overlap their opponents frequently and adjust their behavior in subsequent territorial encounters (Otter et al., 1999; Peake et al., 2002, 2001; Toth et al., 2012) or reproductive decisions (Garcia-Fernandez et al., 2010; Mennill et al., 2002).

Moreover, eavesdropping on such interactions may be necessary for vocal learning in juvenile songbirds (Templeton et al. 2010; but see Mennill et al. 2019). However, what precisely a high level of matching and overlapping signals about a male and whether males that match and overlap frequently are, for example, more successful in territory defense, or of higher social status is still not well understood. Likewise, it is still unclear whether females may benefit from mating with males that match or overlap more frequently (i.e., have a higher reproductive success).

\section{Extra-territorial forays - looking for another mate?}

In many territorial species, where animals defend resources such as food, mates or breeding places, individuals also make movements beyond their territory boundary ("extra-territorial forays") (Celis-Murillo et al., 2017; Mayer et al., 2017; Messier, 1985; Naguib et al., 2001; Young and Monfort, 2009). Such forays could serve multiple, non-exclusive purposes. A function for extra-territorial forays that has often been suggested is the search for potential extra-pair mates. Paternity testing using genetic markers showed that extra-pair paternity occurs in many pair-bonded species (Brouwer and Griffith, 2019; Larmuseau et al., 2019; Uller and Olsson, 2008). Both males and females can potentially benefit from extra-pair mating: males may gain a fitness benefit as they can sire more offspring without providing additional parental care, whereas females may benefit from ensuring fertilization of eggs, mating with a higher quality or more compatible male, increasing genetic diversity in offspring, or if they can secure additional resources from their extra-pair mate or simply avoid costs arising from male 
harassment by copulating with multiple males (reviewed in Brouwer \& Griffith, 2019; Jennions \& Petrie, 2000; Kempenaers \& Dhondt, 1993). In territorial species, either the male, the female or both have to leave their territory to encounter potential extra-pair mates. In birds specifically, many studies investigated the link between movements beyond territory boundaries and extrapair paternity patterns for both males and females: whereas some related female (Chiver et al., 2008; Double and Cockburn, 2000) or male (Kleven et al., 2006; Schlicht et al., 2015) foraying activity to the occurrence of extra-pair offspring, others did not report such a relationship (CelisMurillo et al., 2017; Stutchbury et al., 2005; Westneat, 1993). In some species, males forayed particularly to territories of nest building or fertile females (Churchill and Hannon, 2010; Currie et al., 1998; Krokene et al., 1996; Pedersen et al., 2006; Stutchbury, 1998; Westneat, 1993) and most often when their mate was not fertile (Akçay et al., 2012; Churchill and Hannon, 2010; Currie et al., 1998; Evans et al., 2008; Ward et al., 2014). This would be expected if males foray to search for extra-pair mates and stay on their territory when trying to avoid being cuckolded. However, other studies report no link of male foraying activity to either the fertility of their own (Celis-Murillo et al., 2017; Pitcher and Stutchbury, 2000; Schlicht et al., 2015) or the visited females (Schlicht et al., 2015). Evidence from studies on Hooded warblers (Wilsonia citrina) shows that males might not face a trade-off between foraying and other activities, as male foraying activity was not correlated with parental investment (nestling provisioning) (Pitcher and Stutchbury, 2000) or within-pair fertilization success (Stutchbury et al., 2005). In some species, females forayed off-territory more often during the time they are fertile (Double and Cockburn, 2000; Neudorf et al., 1997; Pedersen et al., 2006; Ward et al., 2014), which would be expected if females are making forays in search of extra-pair copulations. However, in other species, there was no link between female foraying activity and fertility (Schlicht et al., 2015) or females forayed most often before being fertile (Akçay et al., 2012; Celis-Murillo et al., 2017). Some studies suggested that females may foray at particular times to avoid detection by their mates, such as just before dawn (Double and Cockburn, 2000) or during the night (Ward et al., 2014), whereas in other species no forays at dawn were detected (Akçay et al., 2012) or females forayed most often in the later morning and afternoon, rather than at dawn (Schlicht et al., 2015). In some species, females forays were most often brief visits to neighboring territories during which extra-pair copulations were sometimes observed (Kempenaers et al., 1992; Sheldon, 1994; Smith, 1988). Few studies have investigated whether females are more likely to foray into territories of more elaborately ornamented males. In the hooded warbler, females were more likely to foray when their social mate had a low song rate, but males whom they visited during forays did not have higher song rates than social mates and 
were not more likely to have extra-pair offspring. Still, males who ended up having extra-pair offspring sang at higher rates than the respective social mates, indicating that females might have sampled males during forays even when not particularly foraying to males with high singing activity (Chiver et al., 2008). In the common yellowthroat (Geothlypis trichas) both male and female forays were related to male plumage ornaments: females forayed mostly to territories of males with larger black facial masks than their social mate, whereas males most often visited territories of other males with smaller masks (Pedersen et al., 2006). Overall, it remains unclear whether males and females use extra-territorial forays to search for and assess potential extra-pair mates.

Forays may also serve the search for additional food or collection of social and environmental information that may aid in the selection of future habitat: Yearling wolves (Canis lupus), for example, are more likely to foray off-territory in time of low prey abundance (Messier, 1985) and collared flycatchers (Ficedula albicollis) observe provisioning behavior of other pairs to assess their reproductive success (Doligez et al., 2004a, 2002). However, forays may also be costly, as moving through unfamiliar areas can lead to higher predation risk (Larsen and Boutin, 1994; Metzgar, 1967), higher physiological stress levels (Young and Monfort, 2009) or loss of stored food to pilfering and a higher risk of offspring predation (Gerhardt, 2005; Schmidt and Whelan, 2005). These risks and costs may not be the same for different individuals and thus individual foraying activity may vary depending on sex and body size (Schlicht et al., 2015), age (Celis-Murillo et al., 2017; Kleven et al., 2006; Messier, 1985), and social status (Mayer et al., 2017). Individuals may also vary in their foraying behavior due to consistent behavioral differences (personality) associated with the likelihood to take risks. In many species, some individuals are consistently more aggressive, bolder or more exploratory than others, and these differences prevail across context or time (Sih et al., 2004; van Oers and Naguib, 2013). Personality differences are important for explaining migratory tendency, dispersal or foraging behavior (Boon et al., 2008; Chapman et al., 2011; Cote and Clobert, 2007; Nilsson et al., 2010; Wilson and McLaughlin, 2007), the use of social and environmental information (Kurvers et al., 2010; Smit and van Oers, 2019; van Oers et al., 2005; van Overveld and Matthysen, 2013), social connectivity (Aplin et al., 2013; Godfrey et al., 2012; Picq et al., 2019; Snijders et al., 2014) and extra-pair paternity (Patrick et al., 2012; van Oers et al., 2008). In territorial populations, higher social connectivity or occurrence of extra-pair paternity may thus, at least partly, be explained by personality effects on the foraying behavior, and thus encounter rates, 
of individuals. Personality may thus be an important driver that influences the off-territory movements of both females and males.

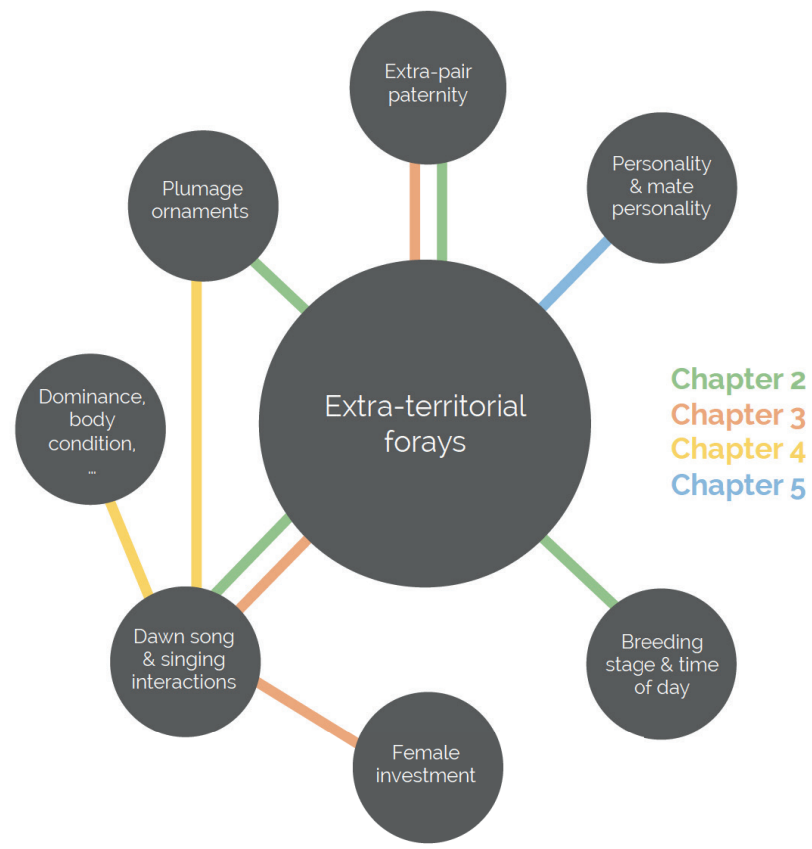

Figure 1 - Conceptual overview of the thesis. To understand the proximate and ultimate factors shaping complex communication systems like bird song, it is important to obtain information of participating individuals and their response on different levels and at different times. Combining automated radio-tracking, (array-) recordings, interactive playbacks and microsatellite analysis I quantified signal production by senders (at dawn in Chapter 2 and in interactions during the day in Chapter $3 \& \& 4)$ and their association with signaler characteristics (Chapter 4) that may be important to receivers. I determined responses of receivers in their spatial (Chapter $2 \boldsymbol{\&} 3$ ) and reproductive investment behavior (Chapter 3) and investigated links between spatial responses and extra-pair mating behavior (Chapter 2 \& 3). I investigated important additional factors that can influence a receiver's spatial behavior, such as consistent individual behavioral differences (personality) (Chapter 5) and timing (Chapter 2).

\section{Thesis aim and outline}

The overall aim of this thesis is to explore the role of male singing behavior at dawn and in male-male interactions during the day in spatial behavior ("extra-territorial foraying") and reproductive decision making in the great tit. In Chapter 2, I examine at what times during the day and the breeding period females and males move off-territory, whether these movements are linked to male dawn song behavior or plumage ornaments and whether they are associated with extra-pair paternity. In Chapter 3, I use an interactive playback design to experimentally test whether eavesdropping on competitive male-male singing interactions influences male or female foraying behavior or female reproductive investment. Using an array of programmable 
recorders, I monitor natural, not playback induced singing interactions between males in Chapter 4 to study whether asymmetries in the use of specific singing patterns during male song contests predict the relative difference between males in several characteristics that might be important for eavesdroppers to assess rivals and potential mates. As the occurrence of extrapair offspring, social connectivity, and the use of social information has previously been linked to individual personality in the great tit, I examine in Chapter 5 whether personality may be an important factor associated with foraying behavior in this species. Finally, in Chapter 6, I summarize the findings of this thesis, discuss them in a broader context, and conclude with outlining some knowledge gaps that may be interesting to pursue in future studies. 




\section{CHAPTER 2 \\ Extra-territorial forays by great tits are associated with dawn song in unexpected ways}

Nina Bircher, Kees van Oers, Camilla A. Hinde, Marc Naguib

Published in Behavioral Ecology (2020) 


\section{Abstract}

Conspicuous male signals often play an important role in both attracting mates and deterring rivals. In territorial species with extra-pair mating, female and male forays to other territories may be an important component underlying female choice and male mating success and might be influenced by male advertisement signals. Yet, whether off-territory foraying is associated with male signals is still not well understood. Here, we tested how female and male forays are associated with short-range visual and long-range acoustic signals (dawn song). We used an automated radio-tracking system to follow the movements of wild great tits (Parus major) to other territories in relation to male dawn song, plumage ornaments, and extra-pair paternity. We show that both sexes frequently forayed into others' territories throughout the breeding period. Movements of both males and females were associated with male song, but not with plumage ornaments. Contrary to our expectations, females stayed away from territories where males sang elaborately, while males were attracted to those territories. Moreover, neither female nor male forays were associated with the occurrence of extra-pair offspring. Our results thus suggest that while forays into other territories are associated with male dawn song, females may not be attracted and males not repelled by dawn song. This sheds a different light on the sexspecific effects of male advertisement signals, expanding the view on the selection pressures shaping such communication systems. 


\section{Introduction}

The conspicuous signals of males in many animal species often serve a dual function in both intersexual selection and intrasexual competition (Berglund et al., 1996). These signals allow both females and males to gain important information on the motivation and quality of the signaler (Behr et al., 2006; Fischer et al., 2004; Gil and Gahr, 2002; Hill and Farmer, 2005), often using several different sensory modalities (Candolin, 2003; Hebets and Papaj, 2005). Acoustic signals, such as vocalizations, can be detected by receivers at greater distances than for example visual signals, like color ornamentation (Hebets and Papaj, 2005). Using such longrange signals, receivers can assess a signaler from a distance without risking potentially costly physical interactions (Naguib and Wiley, 2001; Oliveira et al., 1998; Peake et al., 2002). Thus, acoustic signals play a key role in facilitating or discouraging close range associations between individuals and will affect whether or not information from short-range signals will be accessible (Snijders and Naguib, 2017). In territorial species like for example several wellstudied songbirds, the conspicuous advertisement signals of males have been shown to repel other males (Krebs et al., 1978; Nowicki et al., 1998; Snijders et al., 2017a), while attracting females (Chiver et al., 2008; Snijders et al., 2017a). Specifically the high singing activity at dawn ("dawn song") that males engage in throughout the breeding season may play an important role in keeping away territory intruders and attracting (extra-pair)-mates (Gil and Llusia, 2020; Staicer et al., 1996). However, our understanding of the behavioral responses of males and females in relation to long range signaling stems mostly from playback studies focusing on immediate spatial responses or from correlational studies linking male song traits to reproductive success (Catchpole and Slater, 2008). Especially in species with extra-pair mating, where either the female or male has to foray off-territory ("extra-territorial foray") in order to search for potential extra-pair mates (Kempenaers et al., 1992; Schlicht et al., 2015), the behavioral response to male singing is still not well understood (Bircher and Naguib, 2020). Both females and males have been shown to engage in extra-territorial foraying (Akçay et al., 2012; Celis-Murillo et al., 2017; Kleven et al., 2006; Neudorf et al., 1997). Such forays could have multiple, non-exclusive functions: Individuals may foray into other territories to assess, for example, territory quality and breeding success to optimize their own future reproductive and settlement decisions (Doligez et al., 2004b; Ward, 2005), or in search for food and nesting material (Westneat, 1993). There is evidence in some species that females foray more often when they are fertile (Chiver et al., 2008; Double and Cockburn, 2000; Neudorf et al., 1997), indicating that these forays might be used to copulate with extra-pair mates. Evidence from radio-tracking (Kleven et al., 2006) and from visits to other nest boxes recorded with pit tags 
(Schlicht et al., 2015), shows that male foraying effort predicts the occurrence of extra-pair offspring and is correlated with success in obtaining extra-pair fertilizations. Males in some species also forayed more often into other territories when females are fertile (Akçay et al., 2012; Pedersen et al., 2006), suggesting that they actively seek out potential extra-pair mates when foraying. However, foraying could also be costly for both males and females as it may lead to aggressive encounters with territory owners (Dale and Slagsvold, 1995). Moreover, females may risk losing parental care by their social mate when engaging in extra-pair copulations while males may face a trade-off between foraying in pursuit of extra-pair copulations and mate guarding to avoid being cuckolded (Westneat and Stewart, 2003). Thus, in territorial species with extra-pair mating, females could use long-range signals such as dawn song to decide from a distance where to foray. In some species female forays take place specifically during a time of day with high male singing activity, which would allow females to sample male song particularly well (Double and Cockburn, 2000; Roth et al., 2009). Female black-capped chickadees were observed to sometimes copulate with extra-pair mates during twilight at dawn (Mennill et al., 2004) and in great tits, females with extra-pair young emerged earlier from their nest boxes at dawn compared to females without extra-pair offspring just before egg laying (Halfwerk et al., 2011), suggesting that the time at dawn might be important for extra-pair behavior. Similarly, males might use acoustic or visual signals to assess the fighting ability or dominance of other males and decide where there is a better chance to cuckold the territory owner. To date, most studies on foraying behavior have focused on either males or females, were restricted due to time consuming behavioral observations and manual radiotracking or did not include both acoustic and visual traits that may play a role in prospecting behavior.

Here we took a comprehensive approach to determine the relation between male and female extraterritorial forays and male signals, integrating both acoustic and visual signaling and genetic analyses using wild great tits (Parus major) as a model species. We quantified male vocal (dawn singing) and plumage (yellowness and size of the black breast stripe) ornaments. We used an automated tracking system to track male and female spatial behavior continuously throughout the breeding season to determine how foraying associates with male signaling and whether or not it is connected to the occurrence of extra-pair offspring. We determined throughout the whole breeding season a) the frequency and number of males and females intruding into another territory and b) the time males and females spent on another territory in relation to the resident male's song and plumage traits. We expected that females foray mostly 
when they are fertile and are attracted to territories of males with more elaborate song traits. Conversely, we predicted males to stay away from territories of other males with more elaborate signals and to foray less often during the time their mate is fertile to avoid getting cuckolded. We expected acoustic, long-range signals (dawn song) to be more important than visual, shortrange signals in attracting or repelling other individuals from territories. Finally, we predicted that broods of females that are foraying frequently, of males that are foraying frequently when their mate is fertile (and therefore would mate guard less), and broods in territories that are often intruded by other males, are more likely to contain extra-pair offspring.

\section{Materials and methods}

\section{Study site and general field methods}

This study was conducted in 2016 in Westerheide, a mixed deciduous forest near Arnhem, The Netherlands with approximately 100 breeding pairs of great tits breeding in nest boxes per year. From late March to late June, we checked nest boxes about once per week to determine the start of egg laying, start of incubation, hatching date and fledging success. We caught parents during chick rearing using spring traps when chicks were 10 days old (hatching $=$ day 0 ) and measured weight, length of tarsus and wing length (length of the third primary; P3) to the nearest mm. We collected two blood samples of approx. $10 \mu \mathrm{l}$ from the brachial vein from each parent. When chicks were 14 days old, we ringed them with a unique aluminum ring and measured tarsus length and weight. We collected one blood sample of approx. $10 \mu$ from the brachial vein from each chick.

\section{Radio tracking and quantifying forays}

We used an automated radio-tracking system called "Encounternet" (Encounternet LLC, Portland, USA) to continuously track movements of males and females during the breeding season (Mennill et al., 2012b; Snijders et al., 2017a, 2014). Encounternet consists of roaming nodes (tags) transmitting an individual ID code every 5 seconds and base nodes (receivers), that were distributed throughout the study site. Receivers store the ID code, time stamp, and signal strength (Received Signal Strength Indication - RSSI) value for every signal within up to $90 \mathrm{~m}$ from the transmitter. On the $22^{\text {nd }}$ of March 2016 we tagged 79 birds (41 females and 38 males). Birds were caught while roosting in their nest boxes and equipped with a radio tag of approximately $1.2 \mathrm{~g}$ using a leg loop harness, then released back to their nest box (Snijders et 
al., 2017b). We received permission for all bird handling procedures in this study by the Dutch legal entity Dier Experimenten Comissie (DEC).

We placed Encounternet-receivers up to $5 \mathrm{~m}$ from nest boxes at a height of approximately $2 \mathrm{~m}$ to monitor tagged birds entering the area around a nest box. In the area we monitored, 58 nest boxes were used by great tit pairs for breeding, but due to technical difficulties with some of the receivers, data were collected for only 38 territories. We used signal strength values (RSSI) stored by receivers to estimate the distance between detected tags and receivers (Mennill et al., 2012b) and subsequently excluded all logs that were determined to be further away from a nest box than $15 \mathrm{~m}$. We estimated the cut-off signal strength value at $15 \mathrm{~m}$ using a RSSI-distance regression based on measures from nine calibration transects conducted at different areas within the study site. To do so, we positioned tags at six different distances $(2,10,20,30,40$ and 50 $\mathrm{m}$ ), three different heights (ground level, 2 and $6 \mathrm{~m}$ ) with different antennas angles and either moved them slightly or held them still, to simulate birds in different positions. We calibrated all receivers before deployment to account for between receiver variation in detection sensitivity. For details on the calibration of the radiotracking system, see the supplementary material. We choose a radius of $15 \mathrm{~m}$ so we could assign a received signal to a nest box (the smallest distance between neighboring boxes in our study area was $30 \mathrm{~m}$ ). Using a sliding window approach, we treated a bird as present near a nest box as long as its tag was logged by the respective receiver at least three times during any 30 second time window (i.e. the receiver logged $50 \%$ of the signals sent out by the tag in 30 seconds). This way we excluded single logs resulting from birds just passing through the territory. Following this rule, we considered any time a bird was present near a nest box that was not its own breeding box as an extra-territorial foray. We were thus able to detect whether, when and for how long tagged individuals were present in the vicinity of another pair's breeding area. However, our data does not provide information about which other individuals they have close encounters with (for example whether foraying intruders ever approach the opposite sex member of the resident pair).

Of the 79 birds tagged in March, 59 (31 females and 28 males) also bred in our study site, 49 of them in the area covered by receivers. We removed tags when the tagged birds' chicks were 14 days of age. Tagged birds were tracked for an average of $31 \pm 20$ days. We used data obtained from March until the end of May, thus covering the majority of the breeding period, only excluding the day following tagging. For analysis, we divided the tracking period into five stages relative to the onset of egg laying (day 0): Pre-fertile (from the beginning of tracking until day -8 , fertile (the week before the first egg from day -7 until day -1) (Birkhead, 1992), 
egg laying (day 0 until the last egg), incubation (day after last egg until hatching) and nestling stage. During the nestling stage we monitored only the first $5 \pm 4$ days. Due to technical difficulties with some receivers, the other stages (pre-fertile, fertile, egg laying and incubation) were fully monitored only for 23 nest boxes, but for all 38 boxes at minimum the egg laying stage was fully covered. We divided the day into early morning (5:00 - 7:00; which includes the twilight period before sunrise when males are singing), morning (7:00 - 12:00), afternoon (12:00 - 18:00) and evening (18:00 - 20:00). We excluded visits logged during the later evening and night $(20: 00-5: 00)$ as they seemed to be mostly caused by birds sleeping close to other boxes during the pre-fertile period.

\section{Acoustic recordings and measurements}

We recorded male dawn song on several days during the fertile period of their mate using timeprogrammed Song Meter SM3 recorders (Wildlife Acoustics, Inc. Maynard, MA, USA) mounted above the nest boxes. We selected the best quality recording for each male (highest signal-to-noise ratio) for analysis. All recordings selected for analysis were recorded just before egg laying or during early egg laying (day -3 to day 4 with day 0 being the day of the first egg laid). Recordings were analyzed in Avisoft-SASLab Pro version 5.2.10 (Avisoft, Berlin, Germany). We measured the start and end time of the dawn song in minutes before sunrise, the average song duration (seconds), song rate (songs / minute), dawn song duration (minutes), proportion of time spent singing, and the repertoire size for the entire dawn song. All six measures have previously been suggested to indicate male quality or be associated with female choice in great tits or other species (Gil and Gahr, 2002; Hasselquist et al., 1996; Poesel et al., 2006). We defined the start of the dawn song as the time a bird sang its first song and the end as the time when a bird stopped singing for longer than 7 min (Naguib et al., 2019). To determine repertoire size we followed existing song type categorization criteria for great tits (McGregor et al., 1981). All measures were taken by the same person, who was blind to other information about the individual (tracking data or paternity results) at that point. Since recordings were made with automated, stationary recorders, our analysis is restricted to the part of the dawn song a male sang in the vicinity of the nest box. We recognize that by only measuring song characteristics from one day, we do not take within individual variation in singing behavior during the season into account. However, other studies have shown that single dawn song recordings can provide important information about the singer (see for example Otter et al. 1997; Poesel et al. 2006). Moreover, the start of dawn song and song rate were repeatable between the early and late egg laying stages in the same study population (Snijders 
et al., 2015) and start of dawn song and repertoire size did not differ between some breeding stages (before and during egg laying; Naguib et al. 2019).

Males started their dawn song on average $30.6 \pm 1.4$ min (mean \pm se) before sunrise and sang for $28.2 \pm 2.4 \mathrm{~min}$ (mean $\pm \mathrm{se}$ ) with a mean song rate of $6.4 \pm 0.4$ songs (mean $\pm \mathrm{se}$ ) per minute, song duration of $2.3 \pm 0.09 \mathrm{~s}$ (mean $\pm \mathrm{se}$ ), repertoire size of $3.1 \pm 0.3$ song types (mean $\pm \mathrm{se}$ ) and proportion of time spent singing $32.2 \pm 1 \%$ (mean \pm se). We performed a principal component analysis (PCA) using IBM SPSS Statistics v. 23.0 (IBM Corp., 2013, Armonk, NY, USA) with Varimax rotation and Kaiser Normalization, including all three principal components with eigenvalues $>1$. For further analysis, we used the first three components which accounted for $82 \%$ of the total variation in the six song measures. PC1 was positively associated with dawn song duration, start time of the dawn song and repertoire size. Males with a high PC1 score therefore sang for longer at dawn, started their dawn song earlier (as we measured start time in minutes before sunrise so that higher values indicate an earlier start) and sang a larger number of different song types during their dawn song. PC2 was positively associated with the proportion of time spent singing and song rate, thus males with a high PC2 score sang more during their dawn song. PC3 was positively associated with mean song duration, thus males with high PC3 scores sang on average longer songs during the dawn chorus (see Table 3 for all factor loadings on PC1, PC2 and PC3).

Table 3 - PCA loadings of song measures. PC1 was positively associated with dawn song duration (factor loading $=0.88)$, start time of the dawn song (factor loading $=0.81)$ and repertoire size (factor loading $=0.78)$. PC2 was positively associated with the proportion of time spent singing (factor loading $=0.90$ ) and song rate (factor loading $=0.81$ ). Mean song duration was positively associated with PC3 (factor loading 0.98).

\begin{tabular}{lccc}
\hline & PC1 & PC2 & PC3 \\
\hline Dawn song duration & $\mathbf{0 . 8 8}$ & -0.42 & 0.10 \\
Start time of dawn song & $\mathbf{0 . 8 1}$ & -0.24 & 0.17 \\
Repertoire size & $\mathbf{0 . 7 8}$ & -0.28 & -0.11 \\
Proportion of time spent singing & 0.12 & $\mathbf{0 . 9 0}$ & 0.37 \\
Song rate & -0.27 & $\mathbf{0 . 8 1}$ & -0.46 \\
Mean song duration & 0.03 & 0.06 & $\mathbf{0 . 9 8}$ \\
\hline
\end{tabular}




\section{Plumage measurements}

When adults were caught during the breeding season, we took a photo of the breast stripe and collected samples of the yellow breast feathers to quantify breast stripe size and yellowness of the plumage for males. Both plumage traits have been suggested to be important indicators of male quality in the great tit (Norris, 1993; Senar et al., 2008). We took photos with a Coolpix L31 camera (Nikon Corporation, Tokyo, Japan) mounted on a camera stand in standardized distance and angle, holding the bird with the crown and legs touching the background on which was a $1 \mathrm{~cm}^{2}$ grid sheet (Figuerola and Senar, 2000). We measured the size of male breast stripes in $\mathrm{cm}^{2}$ from where the ventral stripe widens into a throat patch to the posterior end of the stripe with the image analysis software ImageJ v. 1.45s (Abramoff et al., 2004).

We collected feather samples from the yellow patches from each side of the breast stripe and measured plumage reflectance following the methods in (Quesada and Senar, 2006). The samples of yellow breast feathers consisted of at least 12 feathers ( 6 from each side), a number that has been shown to produce the same color measurements as when measuring directly on the bird (Quesada and Senar, 2006). We stacked the feathers on a black velvet surface, superimposing 4 layers of 3 feathers to imitate the plumage surface of a bird and obtained reflectance spectra using a spectrometer (JAZ, Ocean Optics, Dunedin, FL, USA) with a xenon light source (JAZ-PX, Ocean Optics) and a bifurcated fibre-optic probe. The probe was fitted with a cylindrical probe holder to exclude ambient light and standardize the measuring distance to $0.8 \mathrm{~mm}$ and we held it perpendicular to the feather sample for measuring. We obtained reflectance measures with the program SPECTRASUITE v. 2.0.162 (Ocean Optics) in reference to a white tile surface. In total, we took 9 readings per sample, reshuffling the feathers after every third reading. Each reading itself was the average of 12 scans of $40 \mathrm{~ms}$ duration. Using the program TETRACOLORSPACE v. 1b BETA (Stoddard and Prum, 2008) we then calculated the average photon catch of each color cone type; ultra-violet sensitive or violet (UVS), short-wavelength sensitive or blue (SWS), medium-wavelength sensitive or green (MWS) and long-wavelength sensitive or red (LWS) for the average avian visible spectrum (Stoddard and Prum, 2008). TETRACOLORSPACE uses data on cone sensitivity to model the signal from a bird's perspective. We then used these average cone catches of each sample to calculate its SWS ratio, a measure of intensity of the yellow plumage (Evans et al., 2010), using the formula $3^{-1}(U V S+M W S+L W S) / S W S$.

Extra - pair paternity 
All collected blood samples were suspended in Eppendorf tubes containing $1 \mathrm{ml}$ of Queen's lysis or Cell lysis buffer. We extracted and amplified DNA with the FavorPrep 96-well Genomic DNA Kit (Favorgen Biotech Corporation, Ping-Tung, Taiwan) and the QIAGEN multiplex PCR kit (QIAGEN GmbH, Hilden, Germany) following the manufacturer's protocol. To determine paternity of chicks we used 5 microsatellite markers: PmaTAGAn71, PmaGAn27 PmaTGAn33, PmaC25 and PmaD105 (Saladin et al., 2003) in one multiplex PCR. PCR products were run on an ABI 3130 genetic analyzer (Applied Biosystems, Foster City, CA, USA) with a molecular size standard (GeneScan 500-LIZ, Applied Biosystems). We used GeneMapper v. 4.0 (Applied Biosystems) to determine the sizes of the PCR products and derive the genotype for each individual. We determined whether a chick was within-pair or extra-pair with CERVUS v. 3.0.7 (Marshall et al., 1998) testing all chicks against their putative fathers using the following simulation parameters: $98 \%$ of loci typed, error rate $0.01 \%, 10{ }^{\prime} 000$ cycles and two candidate parents. We treated chicks as extra-pair if there were two or more mismatches with the putative father and the putative father was not the social father according to the analysis in CERVUS (significant trio LOD i.e., logarithm of the odds score). For two nest boxes we did not catch the male during the field season and inferred the putative father genotype based on the genotypes of the chicks and the mother and then used the inferred genotype to identify the father among the tagged males. We confirmed the identity of these two males using the tracking data at the respective nest boxes. Two nests, in which chicks did not hatch or died before sampling, were not included in the analysis. We determined the extra-pair status of 441 out of 443 sampled chicks of 73 broods. For two chicks (in separate nests) we did not have a blood sample. The combined exclusion probability for all microsatellites was $>99.9 \%$. Two of our loci significantly deviated from the Hardy-Weinberg equilibrium when the genotypes of all individuals in the analysis were included (PmaD105: $\chi^{2}=24.81$, df $=10, \mathrm{p}=0.006$, PmaTAGAn71: $\left.\chi^{2}=29.58, \mathrm{df}=10, \mathrm{p}=0.001\right)$. This was likely due to the family structure of the data.

\section{Statistical analyses}

All statistical analyses were done in R v. 3.4.3 (R Core Team, 2019). We analyzed all data with generalized linear models and generalized linear mixed models using the $\mathrm{R}$ packages lme4 (Bates et al., 2015) and glmmTMB (Brooks et al., 2017) and checked model assumptions using diagnostic plots created with the $\mathrm{R}$ package DHARMa (Hartig, 2019). We determined the significance of fixed effects with likelihood ratio tests and used stepwise backwards elimination, starting with the least significant variable, to obtain minimal adequate models. 
Fixed effects used as control factors (lay date relative to the population median and average distance to other boxes included in the tracking study) as well as random factors always remained in the minimal adequate model, independent of significance.

\section{Resident male traits and visiting behavior of males and females}

We quantified visits by females and males as number of forays to a nest box area per hour and number of unique individuals foraying to a nest box area per hour, treating each unique hour as a separate data point. For all hours for which we had no individuals visiting a nest box we added a zero for both number of visits per hour and number of individuals visiting per hour. We then tested whether there is a correlation between the number of visits, the number of visiting individuals and the duration of visits to a nest box (dependent variables) and the traits of the resident male (fixed variables: PC1, PC2, yellowness and breast stripe size). Measures of all four male traits were available for 24 of the 38 boxes with tracking data and we used this subset of our data for all models. We included the average distance to other nest boxes and lay date relative to the population median as fixed factors to account for possible differences in visiting behavior to certain boxes because of their central or edge locations and early or late breeding start. We added nest box ID as random factor to correct for multiple observations per nest box and ran a separate model for female and male visits. We first fitted poisson generalized linear mixed models for both the number of visits and the number of visitors per hour. We tested these poisson GLMMs for over dispersion and zero-inflation using the respective tests provided by the R package DHARMa (Hartig, 2019). Since we found evidence for zero-inflation in the counts of visits to a nest box per hour, we then fitted a zero-inflated poisson GLMM to that data using the R package $\operatorname{lmm} T M B$ (Brooks et al., 2017). We used a poisson GLMM to analyze the number of individuals visiting a nest box per hour and log-transformed visit duration values to use a linear mixed model for the visit duration data.

\section{Timing of forays and traits of foraying individuals}

We quantified the number of forays made by each individual per hour. All hours during which the individual was tracked but no foray was detected were scored as 0 . We then tested whether the number of forays per hour and the duration of forays made by an individual correlated with time of day or the breeding stage (fixed variables). For males, we used the breeding stage of their mate. We knew the breeding stage for every foray for 24 males and 26 females and used the respective data subset for all models. We added lay date relative to the population median and average distance to other nest boxes as fixed variables to control for potential spatial or 
temporal patterns and individual ID as random factor to account for several observations per individual. We first fitted a poisson generalized linear mixed model to the number of forays per hour, but found evidence for zero-inflation using the respective test provided by the $\mathrm{R}$ package DHARMa (Hartig, 2019). We thus used a zero-inflated poisson GLMM to model the number of forays per hour using the R package $\operatorname{glmmTMB}$ (Brooks et al., 2017). We log-transformed visit duration values to use a linear mixed model for the duration data. We tested males and females using separate models. Additionally, we quantified the number of different nest box areas visited by foraying females and males per day: we added a zero to the dataset for each day an individual was tracked, but not detected in any nest box area. We then tested whether the number of areas visited was associated with breeding stage (fixed variable), adding lay date relative to the population median and average distance to other nest boxes as control variables and individual ID as random factor in a poisson generalized linear mixed model, testing males and females separately. Since our results showed that males make the longest forays early in the morning, we subsequently also tested for a relationship between dawn song characteristics (PC1 and PC2) and duration of male forays early in the morning. We used a linear mixed model with either PC1, PC2 or PC3 as fixed factor (in three separate models) and the log-transformed foray duration values as dependent variable. In each model, we controlled for lay date relative to the population median and average distance to other nest boxes and included male ID as random factor.

\section{Extra-pair paternity and forays}

We quantified the average number of forays made per hour by the female and male during the fertile and egg laying stages, the average number of visits by other males received per hour and the occurrence of extra-pair offspring (0/1) for each nest box. Due to the small number of nest boxes with a receiver that had both parents tagged, we used separate binomial linear models to test whether either the forays by the female, the forays by the male or visits by other males predict the occurrence of extra-pair offspring. We included the lay date relative to the population median for each box and the average distance to other boxes as control factors in each model. 


\section{Results}

We collected tracking data of 66 birds (34 females and 32 males) accumulating a total of 30,325 forays (13,995 by females and 16,330 by males) to 38 monitored nest box areas. Visits had an average duration of $66 \mathrm{~s}$ and the longest visit lasted 1.9 hours. Females forayed on average 0.45 $\pm 0.004($ mean $\pm \mathrm{se}$ ) times per hour and $6.73 \pm 0.07$ (mean $\pm \mathrm{se}$ ) times per day. Males made on average $0.59 \pm 0.005($ mean $\pm \mathrm{se}$ ) forays per hour and $10.17 \pm 0.09$ (mean $\pm \mathrm{se})$ forays per day.

Receiving forays

Males with a higher PC1 score received fewer visits by females

Resident males with higher PC1 scores (earlier start, longer dawn song and larger repertoire) received fewer female visits per hour and visits by fewer females per hour (number of visits per hour: $\chi^{2}=8.12, P=0.004$; see Figure 1; number of visitors per hour: $\chi^{2}=7.72, P=0.006, N$ $=20,265$ observation hours, 24 resident males). The resident male's breast stripe size, yellowness, PC2 and PC3 score were not associated with the number of female visits per hour and the number of female visitors per hour (visits per hour: breast stripe size: $\chi^{2}=0.25, P=$ 0.62, yellowness: $\chi^{2}=0.06, P=0.80$, PC2: $\chi^{2}=0.94, P=0.33$, PC3: $\chi^{2}=1.69, P=0.19$; visitors per hour: breast stripe size: $\chi^{2}=0.15, P=0.70$, yellowness: $\chi^{2}=0.05, P=0.82$, PC2: $\chi^{2}=0.72, P=0.40$, PC3: $\chi^{2}=2.48, P=0.12$ ). Males with a high PC3 score (longer song duration) tended to receive shorter visits by females $\left(\chi^{2}=3.29, P=0.07\right)$, but the duration of female visits was not associated with any of the other measured male traits (breast stripe size: $\chi^{2}=0.07, P=0.8$, yellowness: $\chi^{2}=0.15, P=0.7, \mathrm{PC} 1: \chi^{2}=0.54, P=0.46$, PC2: $\chi^{2}=0.06, P$ $=0.8$, PC3: $\chi^{2}=2.58, P=0.11$; see Table 2 and Table S2 and S4 in the supplementary material). 

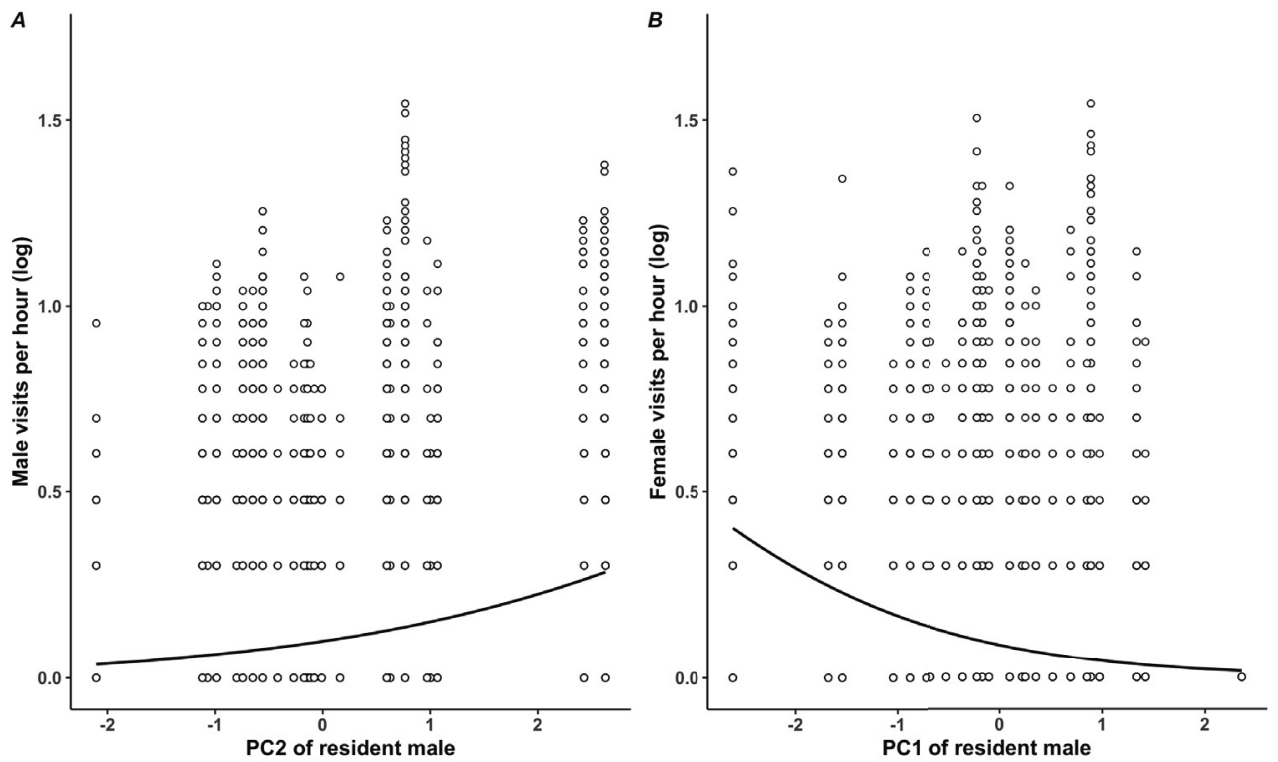

Figure 1 - Associations between song traits (PC1 and PC2) of the resident male and the number of male and female visits to the respective nest box area. Males with a higher PC2 score received more visits by other males. Males with a higher PC1 score received fewer visits by females. We added 1 to counts of visits per hour in order to present them on a log scale. Data includes 20,265 observation hours and 24 resident males, observations with exactly the same values are plotted as one point.

Males with a higher PC2 score received more and longer visits by other males

Resident males with a high PC2 score (higher song rate and proportion of time spent singing) received more and longer visits by more males (visits per hour: $\chi^{2}=5.86, P=0.02$; visitors per hour: $\chi^{2}=5.03, P=0.03 ; N=20,265$ observation hours, 24 resident males; visit duration: $\chi^{2}$ $=12.68, P<0.001 ; N=10,253$ visits, 24 resident males). Resident males with higher PC3 scores (longer song duration) received - and residents with higher PC1 score (earlier start, longer dawn song and larger repertoire) tended to receive - shorter visits by other males (PC3: $\chi^{2}=7.19, P=0.007$; PC1: $\left.\chi^{2}=3.24, P=0.07\right)$, but neither PC3 nor PC1 were associated with the number of visits per hour by other males or the number of males visiting per hour (visits per hour: PC3: $\chi^{2}=1.48, P=0.22 ; \mathrm{PC} 1: \chi^{2}=0.08, P=0.78$; visitors per hour: $\mathrm{PC} 3: \chi^{2}=1.33$, $\left.P=0.25 ; \mathrm{PC} 1: \chi^{2}=0.17, P=0.68\right)$.

Neither breast stripe size nor yellowness of resident males was correlated with the number of male visits per hour, number of male visitors per hour or the duration of visits by other males (number of visits: breast stripe size: $\chi^{2}=0.18, P=0.67$, yellowness: $\chi^{2}=0.04, P=0.85$; number 
of visitors: breast stripe size: $\chi^{2}=0.12, P=0.68$, yellowness: $\chi^{2}=0.02, P=0.88$; duration: breast stripe size: $\chi^{2}=0.01, P=0.92$, yellowness: $\chi^{2}=0.57, P=0.45$, see Table 1 and Table $\mathrm{S} 1$ and $\mathrm{S} 3$ in supplementary material).

\section{Making forays}

Foraying activity of males and females is associated with breeding stage and time of day The number of female forays per hour ( $N=23,655$ observation hours, 26 females) was associated with breeding stage $\left(\chi^{2}=244.84, P<0.001\right)$ and time of day $\left(\chi^{2}=122.52, P<\right.$ 0.001): females made most forays during the nestling stage, whereas they forayed least often in the early morning (see Figure 2). The duration of female forays ( $N=10,667$ forays, 26 females) was associated with time of day $\left(\chi^{2}=81.87, P<0.001\right)$, but not with breeding stage $\left(\chi^{2}=5.78\right.$, $P=0.22$; see Table S5-6 in supplementary material). Females made the longest forays during the early morning (see Figure S4 in supplementary material). The number of different nest box areas visited by females per day also varied between breeding stages $\left(\chi^{2}=180.39, P<0.001\right.$; see Table S9 and Figure S5 in supplementary material): Females visited more different areas during the beginning of the breeding season (pre-fertile stage and during the fertile days before egg laying) than during later stages. 

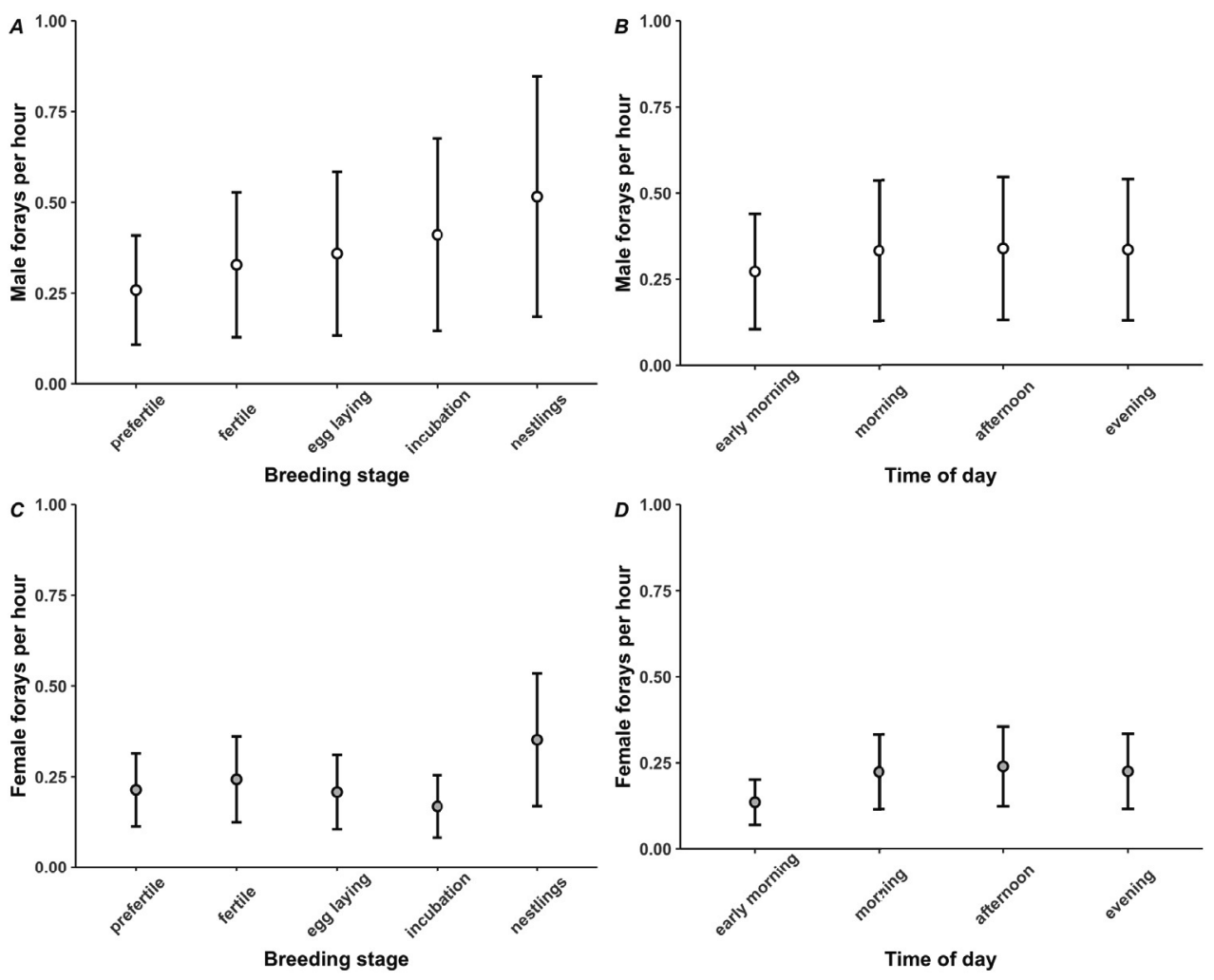

Figure 2 - Foraying activity (number of forays made per hour) of males and females over the breeding season and day. Both males (white) and females forayed least often during the early morning ( $B$ and $D)$ and most often during the nestling stage ( $A$ and $C$ ). Shown are mean model estimates \pm se.

The number of forays made by males per hour was associated with breeding stage and time of day (breeding stage: $\chi^{2}=318.39, P<0.001$, time of day $\chi^{2}=41.02, P<0.001 ; N=21,135$ observation hours, 24 males; see Table S7 in supplementary material). Male foraying activity was lowest during the prefertile phase of his mate and during the early morning (see Figure 2). The duration of male forays was also associated with time of day, but not breeding stage (time of day: $\chi^{2}=24.31, P<0.001$; breeding stage: $\chi^{2}=2.83, P=0.59 ; N=14,419$ forays, 24 males; see Table S8 in supplementary material): Forays were longest in the early morning (see Figure S4 in supplementary material). The duration of male forays in the early morning was not associated with male singing behavior at dawn (PC1: $\chi^{2}=2.01, P=0.16$; PC2: $\chi^{2}=2.20, P=$ $0.14 ;$ PC $3: \chi^{2}=0.089, P=0.77 ; N=781$ forays, 11 males). Moreover, the number of different nest box areas visited by males per day was associated with breeding stage $\left(\chi^{2}=17.0, P=\right.$ 0.002 , see Table S10 in supplementary material), with males visiting the fewer core territories during egg laying and the nestling stage. 
Forays by males and females did not predict occurrence of extra-pair offspring

Extra-pair chicks made up $17.7 \%$ of the chicks sampled and $40 \%$ of broods analyzed contained at least one extra-pair chick. The average number of male visits per hour to a nest box area did not predict occurrence of extra-pair offspring in the visited nest, neither did the average number of forays made per hour by the female or the male of the brood containing extra-pair chicks (male visits: $Z=-0.71, P=0.48$; female forays: $Z=-0.09, P=0.92$; male forays: $Z=0.41, P=$ 0.69 , see Table S11-13 in supplementary material). 
Table 1 - Traits of resident male and number of male visits per hour. Table lists all factors included in a zeroinflated poisson generalized linear mixed model with log link function. The dependent variable was the number of visits per hour to a resident male's nest box area $(N=20,265$ observation hours, 24 resident males) by males. Nest box area was included as random factor (var $\pm s d: 0.99 \pm 0.99)$. Using backward elimination, the estimate and standard error of the last model in which a factor was included are given. The test statistic $\left(\chi^{2}\right)$, degrees of freedom (df) and significance ( $p$ value) given were determined using likelihood-ratio tests (LRT). The factors distance (average distance of a resident male's nest box to other nest boxes) and relative lay date of the resident male's brood were included in the last model, independent of significance.

\begin{tabular}{llllll}
\hline & Estimate & se & $\boldsymbol{\chi}^{\mathbf{2}}$ & df & P value \\
\hline Intercept & -0.13 & 0.21 & - & - & - \\
PC2 & 0.50 & 0.19 & 5.86 & 1 & $\mathbf{0 . 0 2}$ \\
Distance (scaled) & -0.01 & 0.18 & - & - & - \\
Relative lay date (scaled) & -0.13 & 0.21 & - & - & - \\
\hline Dropped terms & & & & 0.85 \\
Yellowness & -0.30 & 1.52 & 0.04 & 1 & 0.78 \\
PC1 & -0.06 & 0.19 & 0.08 & 1 & 0.67 \\
Stripe & -0.07 & 0.16 & 0.18 & 1 & 0.22 \\
PC3 & -0.28 & 0.23 & 1.48 & 1 & \\
\hline
\end{tabular}

Table 2 - Traits of resident male and number of female visits per hour. Table lists all factors included in a zero-inflated poisson generalized linear mixed model with log link function. The dependent variable was the number of visits per hour to a resident male's nest box area $(N=20,265$ observation hours, 24 resident males) by females. Nest box area was included as random factor (var $\pm s d: 1.42 \pm 1.19$ ). Using backward elimination, the estimate and standard error of the last model in which a factor was included are given. The test statistic $\left(\chi^{2}\right)$, degrees of freedom (df) and significance ( $p$ value) given were determined using likelihood-ratio tests (LRT). The factors distance (average distance of a resident male's nest box to other nest boxes) and relative lay date of the resident male's brood were included in the last model, independent of significance.

\begin{tabular}{|c|c|c|c|c|c|}
\hline & Estimate & se & $x^{2}$ & df & P value \\
\hline Intercept & -0.12 & 0.25 & - & - & - \\
\hline PC1 & -0.74 & 0.25 & 8.12 & 1 & 0.004 \\
\hline Distance (scaled) & -0.09 & 0.22 & - & - & - \\
\hline Relative lay date (scaled) & -0.11 & 0.26 & - & - & - \\
\hline \multicolumn{6}{|l|}{ Dropped terms } \\
\hline Yellowness & -0.46 & 1.81 & 0.06 & 1 & 0.80 \\
\hline Stripe & -0.09 & 0.18 & 0.25 & 1 & 0.62 \\
\hline PC2 & 0.21 & 0.22 & 0.94 & 1 & 0.33 \\
\hline PC3 & 0.36 & 0.27 & 1.69 & 1 & 0.19 \\
\hline
\end{tabular}




\section{Discussion}

Our results integrating movement, signaling, and paternity information reveal that forays by both female and male great tits were associated with the dawn song characteristics of resident males, but not with male plumage traits. We show that female forays to other territories were associated with the resident male's repertoire size and the start and duration of its dawn song (PC1). Male forays on the other hand were mainly associated with a male's song rate and the proportion of time spent singing during the dawn song (PC2). In contrast to our expectations, females stayed away from areas where resident males had a larger repertoire, started their dawn song earlier and sang for longer at dawn (high PC1 scores), while males were attracted to areas where resident males had a high song rate and spent a larger proportion of time singing at dawn (high PC2 score). Additionally, females forayed often throughout the whole tracking period and not primarily when they were fertile. Moreover, neither female nor male foraying was associated with the probability of being cuckolded. These findings thus indicate that long-range acoustic (dawn song), but not short-range visual, signals are associated with female and male decisions regarding off-territory movements and thus appear to play an important role in connecting individuals within a territorial neighborhood.

Contrary to our predictions, female great tits forayed less often to territories of resident males with a larger repertoire, earlier and longer dawn song (higher PC1) and tended to make shorter visits to territories of males with a longer song duration (higher PC3). Male song is typically considered to be an honest signal of male quality important in both female attraction and territory defense (Catchpole and Slater, 2008; Gil and Gahr, 2002). Females engaging in extrapair copulations to gain, for example, indirect genetic benefits for their offspring would thus be expected to be attracted to males with more elaborate song traits, such as a larger repertoire (Hasselquist et al., 1996), a more consistent vocal performance (Byers, 2007) or an earlier start of the dawn song (Poesel et al., 2006). Female hooded warblers (Wilsonia citrina) for example were more likely to foray off-territory when their social mate sang at a low rate and preferred extra-pair mates with a higher song rate (Chiver et al., 2008). However, female preference for more elaborate versions of male song traits are not always evident; many field studies, for example, did not find evidence for female preference for larger repertoire sizes (reviewed in Byers and Kroodsma 2009). Moreover, two recent meta studies found no associations between measures of song complexity and reproductive success (Soma and Garamszegi, 2011) and song complexity or output and extra-pair paternity both among and within species (Garamszegi, 2004). More elaborate song traits are often associated with male territory tenure, dominance, 
and willingness to escalate an interaction (Krebs et al., 1978; Otter et al., 1997; Vehrencamp, 2001) and females may not always prefer more dominant males (Ophir and Galef, 2003; Qvarnström and Forsgren, 1998; Wong and Candolin, 2005). Females in the Atlantic molly (Poecilia mexicana) for example showed a preference for superior fighting abilities, but avoided winners after observing male contests, possibly to avoid harassing behavior of these more dominant males (Bierbach et al., 2013). Thus, it may be possible that females use the information conveyed in dawn singing to avoid aggressive males when foraying. This may be beneficial for females especially if they do not foray primarily in search of extra-pair mates, but for other purposes or if they engage in extra-pair mating for reasons other than gaining indirect benefits by mating with higher quality males. Indeed, previous studies in great tits have found no difference between extra-pair mates and social mates in age, body size, survival and the width of the breast stripe, suggesting that gaining indirect benefits may not be a primary reason for extra-pair mating in females (Krokene et al., 1998; Strohbach et al., 1998). Rather, variation in extra-pair paternity (EPP) in this species seems to be connected to consistent behavioral differences between individuals (Patrick et al., 2012; van Oers et al., 2008), that are likely to play an important part in the interactions between female, male and extra-pair male. Female foraying behavior did not predict the occurrence of extra-pair offspring in our study. Moreover, in contrast to other studies (Chiver et al., 2008; Double and Cockburn, 2000; Neudorf et al., 1997), we did not find that females were primarily foraying when they were fertile, which we would have expected if females were foraying mostly to encounter potential extra-pair mates. Even though females also forayed quite substantially during the fertile days just before egg laying, their foraying activity peaked during chick rearing. Additionally, although females also forayed early in the morning, they forayed mostly later during the day. Foraying early in the morning has previously been suggested to be a foraying strategy to avoid detection (Double and Cockburn, 2000) and possibly punishment by the social mate (Westneat and Stewart, 2003). Hence, female forays may serve multiple purposes, such as information gathering in a broader sense or foraging in addition to extra pair mating. That females forayed particularly often when having nestlings may suggest that they foray primarily in search of food. However, on forays during the days before egg laying and during egg laying females could still be prospecting extra pair mates. That females forayed to more different areas during the breeding stages before egg laying might indicate that they are assessing several potential extra-pair mates during this early time in the season before narrowing their search down. However, they could also be gathering other types of information during these early forays, for example on important foraging areas that they could later visit more specifically during chick 
rearing or just move around more before egg laying and incubation. Future studies could test the foraging function experimentally by supplementing females with food and follow the effect on subsequent foraying effort (Humbird and Neudorf, 2008). Moreover, while our data provides almost continuous information on foraying activity of many individuals over the majority of the breeding season, it does not provide information about with whom they interact. More finescale spatial information would allow to determine whether foraying birds actually had closerange encounters with the opposite sex member of the resident pair and whether females had more such encounters on forays they made when fertile, which would be expected if they forayed in search of extra-pair mates.

In contrast to females, males forayed more often and for a longer time to nest box areas of resident males with a high song rate and proportion of time spent singing during the dawn song (high PC2). However, males stayed for a shorter time in areas when the resident male sang longer songs during its dawn song (high PC3) and tended to stay for shorter time when males had a large repertoire size and an early and long dawn song (high PC1). More elaborate singing is often associated with male dominance and stronger territory defense and commonly thought to function as a deterrent to conspecific males (Catchpole and Slater, 2008; Gil and Gahr, 2002). Yet, almost all studies on the keep-out signal function of song are playback studies that simulate an intruder on a territory and measure the immediate response by a territory owner (Catchpole and Slater, 2008). Few studies have actually focused on the more cryptic movements of potential intruders and measured whether the broadcast signal keeps them away (Falls, 1988; Yasukawa, 1981) and which song traits might be particularly important in doing so (Krebs et al., 1978; Nowicki et al., 1998). In European starlings (Sturnus vulgaris), males were actually attracted to nest boxes from which male song was broadcast compared to control boxes with no song. Interestingly, these males still preferentially approached boxes where song of lower complexity was broadcast compared to boxes with more complex song, indicating that singing as such could attract males, but differences in song characteristics could still act as a deterrent (Mountjoy and Lemon, 1991). In great tits, males with a larger repertoire size may be more successful in keeping intruders out (Krebs et al., 1978) and mean song duration is positively correlated with dominance (Lambrechts and Dhondt, 1986). While we found that males stayed for a shorter time in areas when resident males sang longer songs during their dawn song (high PC3) and tended to stay for a shorter time when males had a high PC1 score (which included repertoire size as measure), males did not visit those areas less often. Males indeed might be attracted to territories of very actively singing males (high PC2 score) to gather information on 
territory quality or breeding success for future breeding attempts (Doligez et al., 2004b, 1999) as male singing activity can reflect food availability (Ritschard and Brumm, 2012). Indeed, male foraying behavior in our study was not associated with the occurrence of extra-pair offspring: nests in areas that were visited more by foraying males were not more likely to have extra-pair offspring, nor were males that left their territory more often, more likely to be cuckolded. Moreover, just like females, males showed a peak in foraying activity during the nestling phase, which could indicate that they foray primarily to forage, although males could copulate with fertile females during the whole period. Males made the longest forays early in the morning, which included the time window of twilight before sunrise when they sing their dawn song. However, the duration of those early morning forays was not associated with any of the male signing measures (PC1, PC2, or PC3). Males that sang very actively and for longer in the morning did thus not foray less often during that time, suggesting that males do not tradeoff singing with foraying. Together, these results suggest that males foray primarily for reasons other than to seek extra-pair copulations.

Our findings that females visited territories of males with a larger repertoire size and an earlier and longer dawn song (high PC1) less often and males visited territories of males with a high song rate and proportion of time spent singing (high PC2) more often contrasts the common view on the function of bird song as a territory defense or female attraction signal. While many previous studies showed that male bird song in the short term repels males from approaching (Krebs et al., 1978; Nowicki et al., 1998; Snijders et al., 2017a) and attracts females (Snijders et al., 2017a), we here show the opposite, by integrating singing with movements at other times of the day and over the whole breeding season. Our results thus suggest that male song may influence receiver movements differently at different times of the day and moments during the breeding season, beyond a narrow, immediate time window during or right after the actual singing. It is important to note that the long-term spatial response (forays throughout the breeding season) we measured could also reflect within-individual changes in singing behavior throughout the breeding season that we have not captured with measures from one dawn song. However, previous studies in the same population have shown that at least three of the six dawn song measures we used (song rate, start time of dawn song and repertoire size) were repeatable between some breeding stages (Naguib et al., 2019; Snijders et al., 2015) and other studies have shown that single dawn song recordings can provide important information about the singer (see for example Otter et al. 1997; Poesel et al. 2006). 
Taken together, our results show that males and females made frequent forays to other territories; however, forays were neither associated with plumage traits of males nor the occurrence of extra-pair offspring. Moreover, while females also forayed often during the fertile days before egg laying and during egg laying, the forayed mostly during chick rearing. Our results do thus not provide support for the idea that females use extra-territorial forays to pursue copulations with more elaborately ornamented or singing extra-pair mates, although some of the foraying activity during fertile days could still serve this purpose. Our findings that male dawn song was associated with forays of both females and males, but in the opposite way than shown by studies on more immediate responses to song is surprising and suggests that when including long-term responses to signals, there might be additional effects of birdsong on receivers. Our findings including such long-term responses thus open a new perspective on the role of signaling in spatial and social relations in a population. 


\section{Acknowledgements}

We would like to thank Geldersch Landschap \& Kasteleen for the permission to conduct fieldwork in Westerheide and Mara Ruiz Minano, Maeliss Hoarau, Laura Lute, Tomas Tuvillo and Hongye Zhang for their help with collecting the data. We are grateful to Piet de Goede, Christa Mateman, Martijn van der Sluijs, Lydia Nieuwe Weme and the animal care takers at NIOO-KNAW for their valuable assistance throughout the project, John Burt and Hans Meier for the technical support with the Encounternet system and Liam Bailey for help with the statistical analysis. This study was permitted by the Dutch legal entity Dier Experimenten Comissie (DEC) no. NIOO-10.05 to MN and KvO and no. NIOO 12.02 to KvO. 
Supplement to chapter 2 


\section{Calibration of radio-tracking system}

Received signal strength indication (RSSI) values recorded by Encounternet receivers for each detected tag signal decrease with distance (Figure S3 - A). We used these signal strength values to estimate the distance between detected tags and receivers (Mennill et al., 2012b). To estimate the relationship between distance and received signal strength we placed four tags at different distances along nine transects within the study site. We choose areas of transects so they encompassed differently vegetated areas in our study site. We measured signal strength values along each transect at 2, 10,20,30,40 and 50m distance to the same receiver. We attached tags to a plastic pole and held the pole in position for six minutes at each distance, placing tags at ground level, $2 \mathrm{~m}$ and $6 \mathrm{~m}$ height for two minutes each. To simulate birds in different positions and either moving around or not, we attached tags with their antennas horizontal or perpendicular relative to the receiver's antenna and turned the pole clockwise with approximately 10 turns during the second minute of measuring at each height. On average tags were detected $95 \%$ of the time when within 10 meters and $81 \%$ of the time when within 20 meters of a receiver (see Figure S3 - B). We then used a RSSI-distance regression including all transect measures to predict the signal strength value at $15 \mathrm{~m}$ (obtaining a value of $-3.88 \mathrm{RSSI}$ ) and used this value to exclude logs determined to be further away than $15 \mathrm{~m}$ from the receiver at a given nest box. We choose a radius of $15 \mathrm{~m}$ so we could assign a received signal to just one nest box in most cases (the smallest distance between neighboring boxes in our study area was $30 \mathrm{~m})$.

We calibrated all receivers before deployment to account for between receiver variation in detection sensitivity. We attached four tags to a $2 \mathrm{~m}$ long plastic pole and held the pole at fixed distance of $2 \mathrm{~m}$ to each of the receivers for two minutes. To account for different antenna angles when tags are on birds, we attached tags on the pole with the antennas of two them positioned parallel and of the other two perpendicular relative to the receiver's antenna. We then calculated the mean signal strength logged by each receiver $\left(\operatorname{mean}_{\text {Receiver }}\right)$ and the mean signal strength

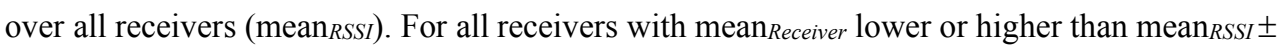
$1 \mathrm{sd}$, we adjusted the signal strength value of all logs during analysis by $\Delta \mid \operatorname{mean}_{\text {RSSI-mean }}$ Receiver $\mid$ to avoid an over or underestimation of the distance between tagged birds and respective receivers. 
Figures
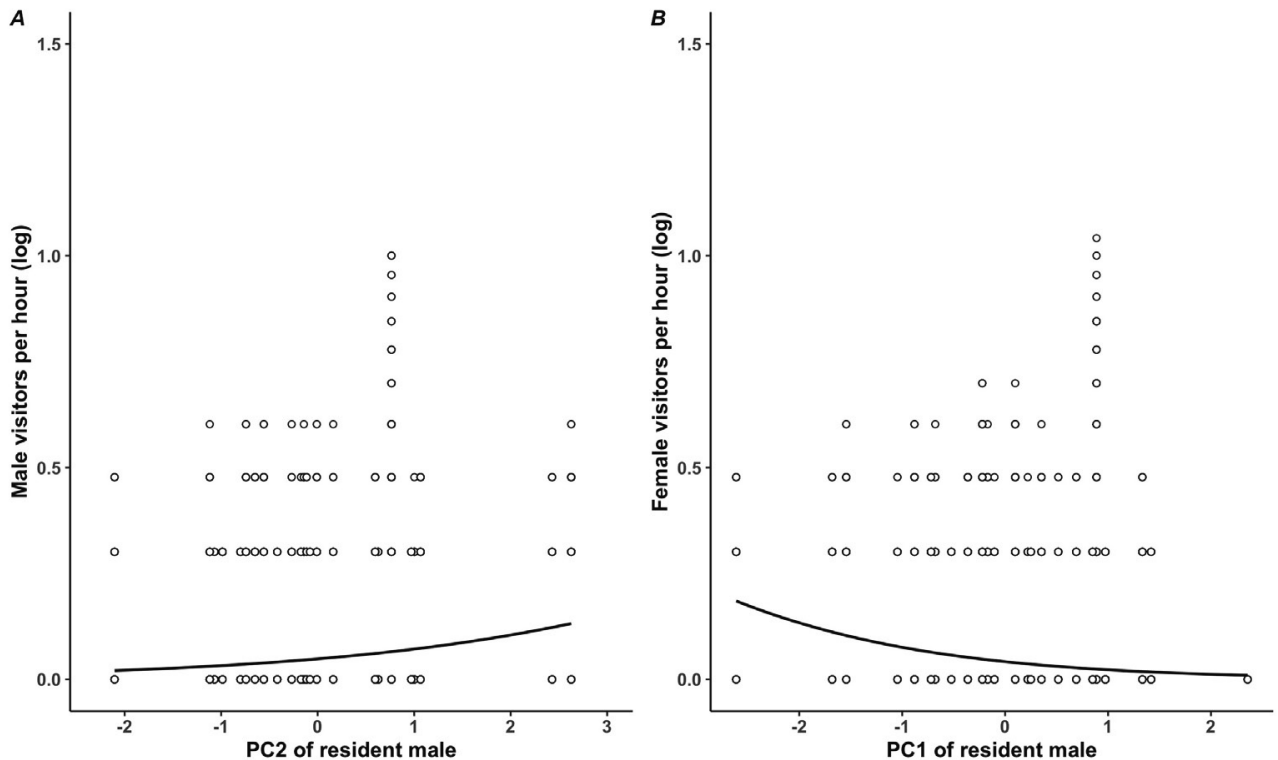

Figure S1 - Associations between song traits (PC1 and PC2) of the resident male and number of male and female visitors to the respective nest box area. Males with a higher PC2 score were visited by more different males. Males with a higher PC1 score received visits by fewer females. We added 1 to counts of visitors per hour in order to present them on a log scale. Data includes 20,265 observation hours and 24 resident males, observations with the same values are plotted as one point. Lines depict model predictions. 

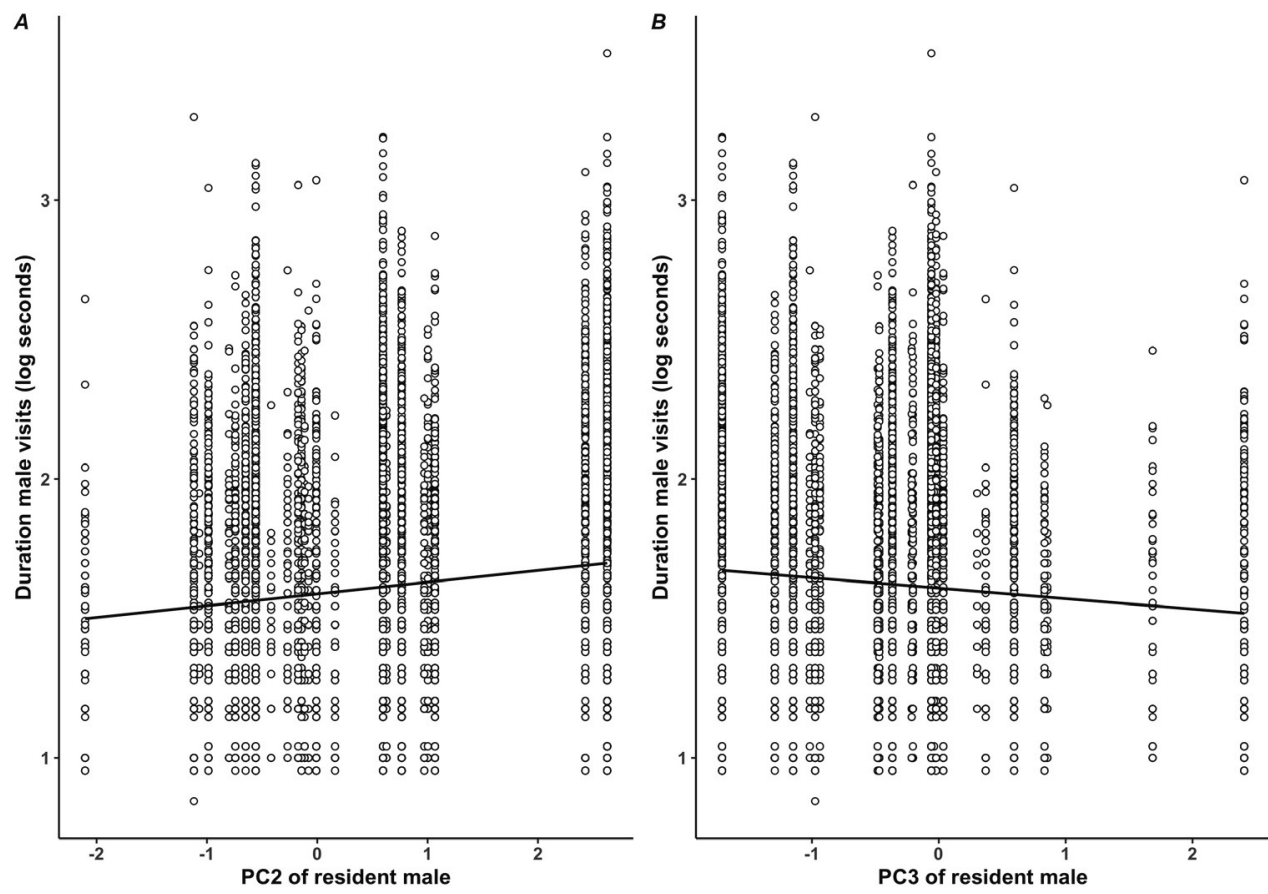

Figure S2 - Associations between song traits (PC2 and PC3) of the resident male and duration of male visits to the respective nest box area. Males with a higher $P C 2$ score received longer visits by other males, whereas males with a higher PC3 score received shorter visits. Data include 10,253 observation hours and 24 resident males, observations with the same values are plotted as one point. Lines depict model predictions. 

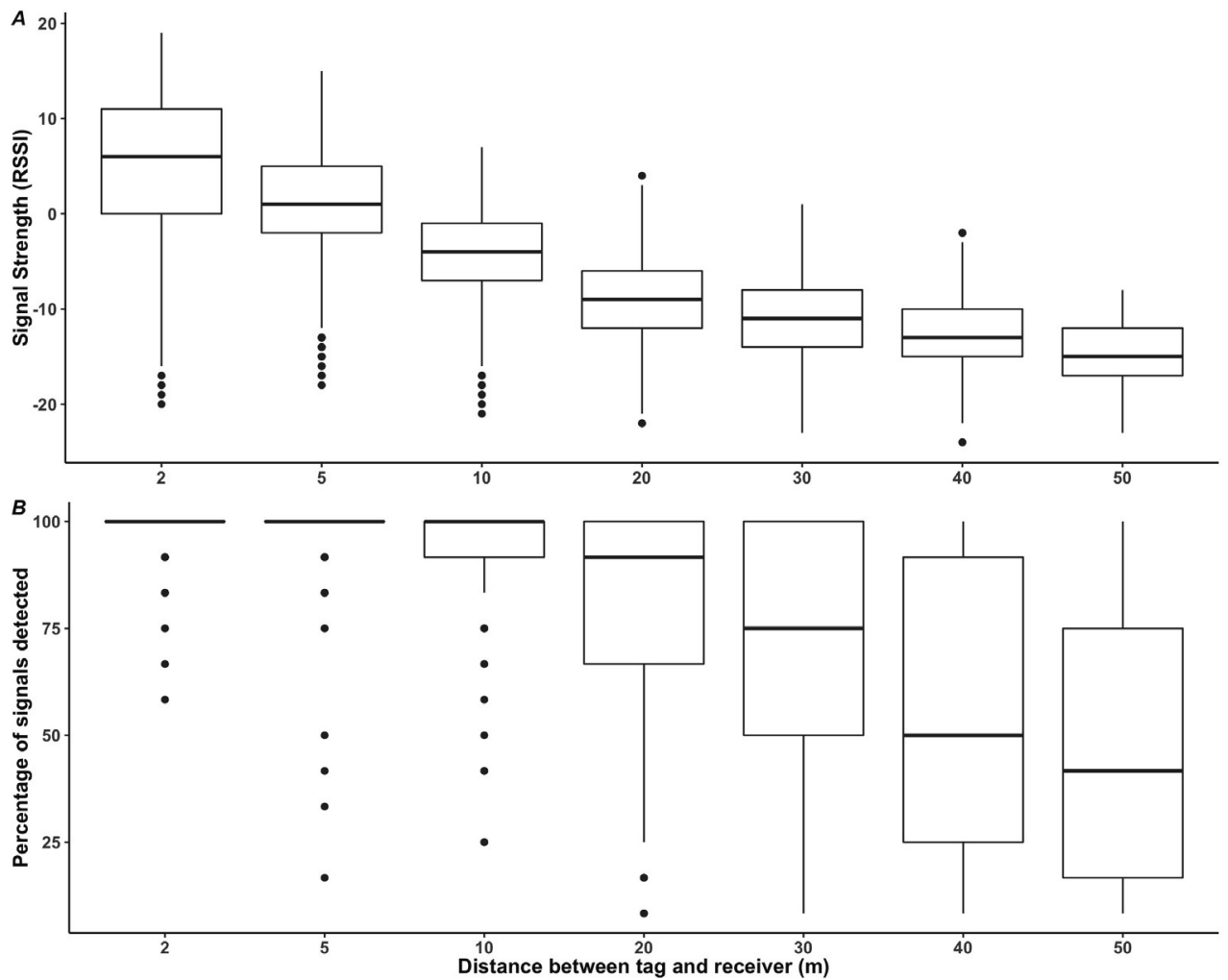

Figure S3 - Signal strength (RSSI), percentage of detected signals and distance between tags and receiver. Signal strength (A) and percentage of detected signals (B) decreased with the distance between tags and receiver. Data include measures from nine different transects with four tags positioned at three different heights (ground level, 2 and $6 \mathrm{~m}$ ), with two different antenna angles (horizontal and perpendicular) and tags either being moved slightly or held still. 

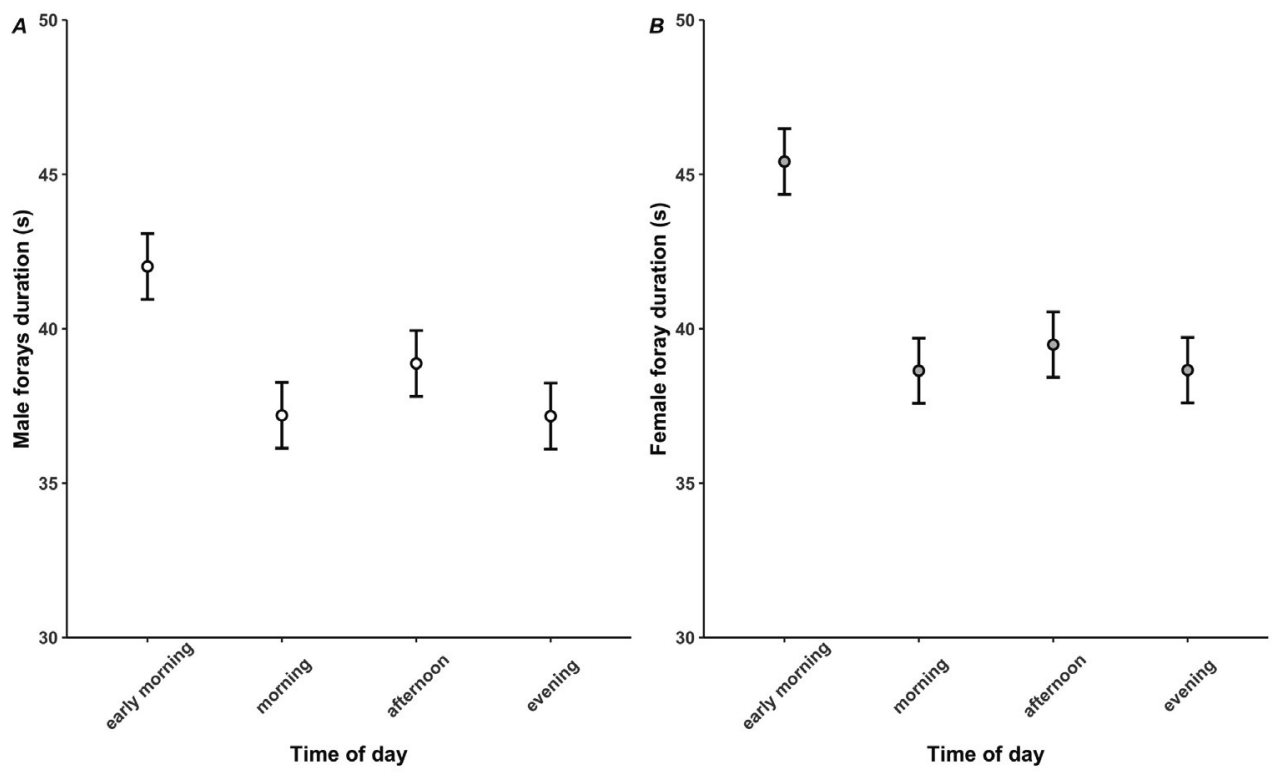

Figure S4 - Foray duration of males and females over the day. Both males (white) and females (grey) made the longest forays early in the morning. Shown are mean model estimates \pm se.
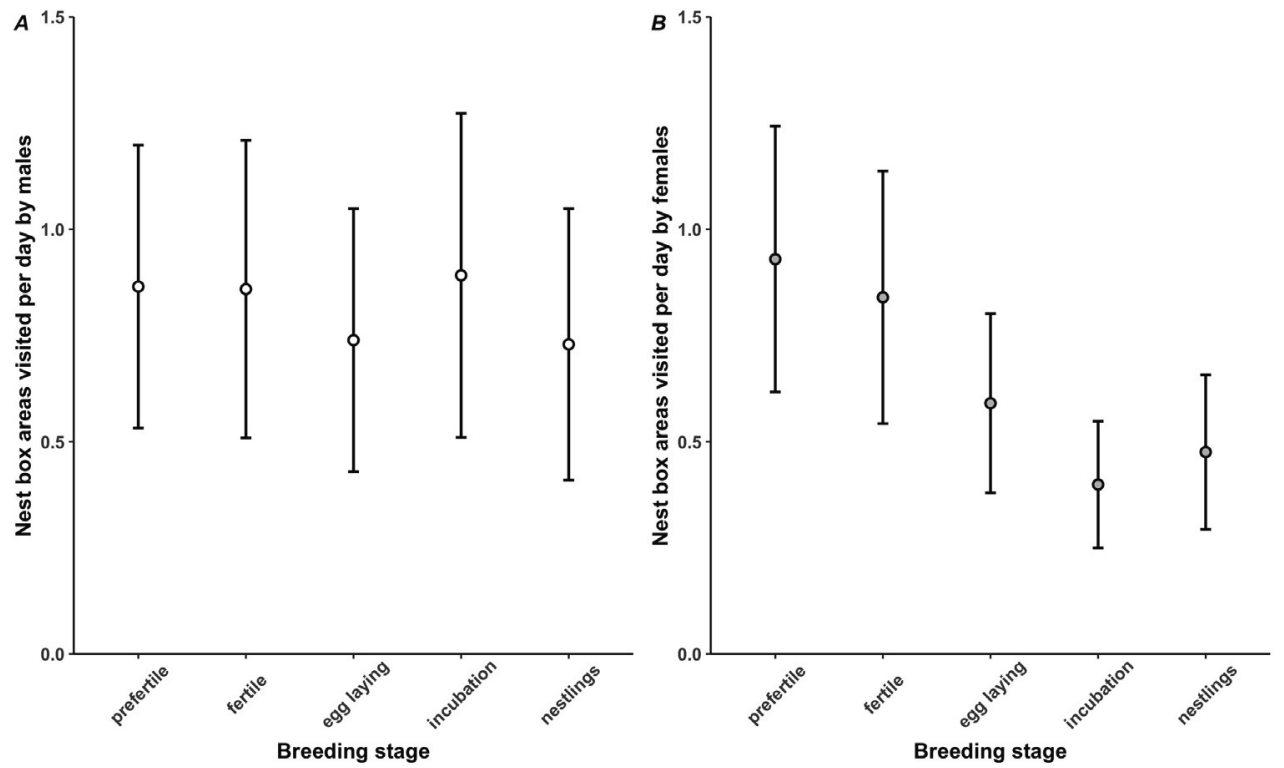

Figure S5 - Number of nest box areas visited per day by foraying males and females over the breeding season. Males (white) visited fewer areas during the egg laying and nestling stage. Females (grey) visited more areas early in the season during the prefertile stage and the fertile days before egg laying (fertile). Shown are mean model estimates \pm se. 


\section{Model tables}

Table S1 - Traits of resident male and number of male visitors per hour. Table lists all factors included in a poisson generalized linear mixed model with log link function. The dependent variable was the number of male visitors per hour to a resident male's nest box area $(N=20265$ observation hours, 24 resident males). Nest box area was included as random factor (var $\pm s d: 0.84 \pm 0.92$ ). Using backward elimination, the estimate and standard error of the last model in which a factor was included are given. The test statistic $\left(\chi^{2}\right)$, degrees of freedom (df) and significance ( $p$ value) given were determined using the likelihood-ratio test (LRT). The factors distance (average distance of a resident male's nest box to other nest boxes) and relative lay date of the resident male's brood were included in the last model, independent of significance.

\begin{tabular}{llllll}
\hline & Estimate & se & $\boldsymbol{\chi}^{\mathbf{2}}$ & df & P value \\
\hline Intercept & -2.14 & 0.19 & - & - & - \\
PC2 & 0.42 & 0.18 & 5.04 & 1 & $\mathbf{0 . 0 3}$ \\
Distance & -0.4 & 0.17 & - & - & - \\
Relative lay date & -0.08 & 0.19 & - & - & - \\
\hline Dropped terms & & & & & \\
Yellowness & -0.03 & 0.20 & 0.02 & 1 & 0.88 \\
PC1 & -0.07 & 0.18 & 0.17 & 1 & 0.68 \\
Stripe & -0.09 & 0.13 & 0.12 & 1 & 0.68 \\
PC3 & 0.41 & 0.17 & 1.33 & 1 & 0.25 \\
\hline
\end{tabular}

Table S2 - Traits of resident male and number of female visitors per hour. Table lists all factors included in a poisson generalized linear mixed model with log link function. The dependent variable was the number of female visitors per hour to a resident male's nest box area $(N=20265$ observation hours, 24 resident males). Nest box area was included as random factor (var $\pm s d: 1.10 \pm 1.05)$. Using backward elimination, the estimate and standard error of the last model in which a factor was included are given. The test statistic $\left(\chi^{2}\right)$, degrees of freedom $(d f)$ and significance ( $p$ value) given were determined using the likelihood-ratio test (LRT). The factors distance (average distance of a resident male's nest box to other nest boxes) and relative lay date of the resident male's brood were included in the last model, independent of significance.

\begin{tabular}{llllll}
\hline & Estimate & se & $\boldsymbol{\chi}^{\mathbf{2}}$ & df & P value \\
\hline Intercept & -1.21 & 2.18 & - & - & - \\
PC1 & -0.64 & 0.22 & 7.72 & 1 & $\mathbf{0 . 0 0 6}$ \\
Distance (scaled) & -2.18 & 4.45 & - & - & - \\
Relative lay date (scaled) & -0.01 & 0.06 & - & - & - \\
\hline Dropped terms & & & & & \\
Yellowness & -0.05 & 0.22 & 0.05 & 1 & 0.82 \\
Stripe & -0.06 & 0.15 & 0.15 & 1 & 0.70 \\
PC2 & 0.39 & 0.22 & 0.72 & 1 & 0.40 \\
PC3 & 0.38 & 0.23 & 2.48 & 1 & 0.12 \\
\hline
\end{tabular}


Table S3 - Traits of resident male and duration of male visits. Table lists all factors included in linear mixed model with the log-transformed duration of male visits to a resident male's nest box area $(N=10253$ observation hours, 24 resident males) as dependent variable. Nest box area was included as random factor (var $\pm s d: 0.002 \pm$ 0.04 , residual variance $\pm s d: 0.16 \pm 0.39)$. Using backward elimination, the estimate and standard error of the last model in which a factor was included are given. The test statistic $\left(\chi^{2}\right)$, degrees of freedom ( $\left.d f\right)$ and significance $(p$ value) given were determined using the likelihood-ratio test (LRT). The factors distance (average distance of a resident male's nest box to other nest boxes) and relative lay date of the resident male's brood were included in the last model, independent of significance.

\begin{tabular}{llllll}
\hline & Estimate & se & $\boldsymbol{\chi}^{\mathbf{2}}$ & df & P value \\
\hline Intercept & 1.57 & 0.08 & - & - & - \\
PC1 & -0.02 & 0.01 & 3.24 & & 0.07 \\
PC2 & 0.04 & 0.01 & 12.68 & 1 & $<\mathbf{0 . 0 0 1}$ \\
PC3 & -0.04 & 0.01 & 7.19 & 1 & $\mathbf{0 . 0 0 7}$ \\
Distance & 0.09 & 0.17 & - & - & - \\
Relative lay date & -0.01 & 0.003 & - & - & - \\
\hline Dropped terms & & & & & \\
Yellowness & 0.03 & 0.09 & 0.01 & 1 & 0.92 \\
Stripe & 0.006 & 0.008 & 0.49 & 1 & 0.48 \\
\hline
\end{tabular}

Table S4 - Traits of resident male and duration of female visits. Table lists all factors included in a linear mixed model with the log-transformed duration of female visits to a resident male's nest box area $(N=9430$ observation hours, 23 resident males) as dependent variable. Nest box area was included as random factor (var $\pm s d: 0.004 \pm 0.06$, residual variance $\pm s d: 0.15 \pm 0.38)$. Using backward elimination, the estimate and standard error of the last model in which a factor was included are given. The test statistic $\left(\chi^{2}\right)$, degrees of freedom (df) and significance ( $p$ value) given were determined using the likelihood-ratio test (LRT). The factors distance (average distance of a resident male's nest box to other nest boxes) and relative lay date of the resident male's brood were included in the last model, independent of significance.

\begin{tabular}{llllll}
\hline & Estimate & se & $\boldsymbol{\chi}^{\mathbf{2}}$ & df & P value \\
\hline Intercept & 1.43 & 0.12 & - & - & - \\
PC3 & -0.03 & 0.02 & 3.29 & 1 & 0.07 \\
Distance & 0.37 & 0.22 & - & - & - \\
Relative lay date & -0.0003 & 0.004 & - & - & - \\
\hline Dropped terms & & & & & \\
Yellowness & .0 .05 & 0.12 & 0.18 & 1 & 0.67 \\
Stripe & 0.004 & 0.01 & 0.14 & 1 & 0.71 \\
PC1 & -0.01 & 0.01 & 0.89 & 1 & 0.35 \\
PC2 & 0.01 & 0.01 & 1.16 & 1 & 0.28 \\
\hline
\end{tabular}


Table S5 - Number of female forays, breeding stage and time of day. Table lists all factors included in a zeroinflated poisson generalized linear mixed model with log link function. The dependent variable was the number of forays undertaken per hour by a female $(N=23,655$ observation hours, 26 females). Female ID was included as random factor ( var $\pm s d: 2.0 \pm 1.40)$. Using backward elimination, the estimate and standard error of the last model in which a factor was included are given. The test statistic $\left(\chi^{2}\right)$, degrees of freedom (df) and significance ( $p$ value) given were determined using the likelihood-ratio test (LRT). The factors distance (average distance of a female's nest box to other nest boxes) and relative lay date of the female's brood were included in the last model, independent of significance.

\begin{tabular}{|c|c|c|c|c|c|c|}
\hline & & Estimate & se & $\chi^{2}$ & df & P value \\
\hline Intercept & & 2.35 & 1.84 & - & - & - \\
\hline \multirow[t]{5}{*}{ Breeding stage } & & & & 244.84 & 4 & $<0.001$ \\
\hline & fertile & 0.21 & 0.05 & & & \\
\hline & nestlings & 0.46 & 0.05 & & & \\
\hline & incubation & -0.29 & 0.05 & & & \\
\hline & prefertile & 0.13 & 0.04 & & & \\
\hline \multirow[t]{4}{*}{ Daytime } & & & & 122.52 & 3 & $<0.001$ \\
\hline & early morning & -0.57 & 0.05 & & & \\
\hline & evening & -0.06 & 0.03 & & & \\
\hline & morning & -0.08 & 0.02 & & & \\
\hline Distance & & -7.33 & 3.60 & - & - & - \\
\hline Relative lay date & & 0.11 & 0.09 & - & - & - \\
\hline
\end{tabular}

Table S6 - Duration of female forays, breeding stage and time of day. Table lists all factors included in a linear mixed model with the log transformed duration of forays undertaken by a female $(N=10,667$ forays, 26 females) as dependent variable. Female ID was included as random factor (var $\pm s d: 0.005 \pm 0.07$, residual variance $\pm s d$ : $0.15 \pm 0.38$ ). The test statistic $\left(\chi^{2}\right)$, degrees of freedom ( $\left.d f\right)$ and significance ( $p$ value) given were determined using the likelihood-ratio test (LRT). The factors distance (average distance of a female's nest box to other nest boxes) and relative lay date of the female's brood were included in the last model, independent of significance.

\begin{tabular}{|c|c|c|c|c|c|}
\hline & Estimate & se & $x^{2}$ & df & P value \\
\hline Intercept & 1.60 & 0.02 & - & - & - \\
\hline \multirow[t]{4}{*}{ Daytime } & & & 81.87 & 3 & $<0.001$ \\
\hline & 0.06 & 0.02 & & & \\
\hline & -0.01 & 0.01 & & & \\
\hline & -0.01 & 0.01 & & & \\
\hline Distance (scaled) & -0.0004 & 0.01 & - & - & - \\
\hline Relative lay date (scaled) & -0.01 & 0.02 & - & - & - \\
\hline \multicolumn{6}{|l|}{ Dropped terms } \\
\hline Breeding stage & & & 5.78 & 4 & 0.22 \\
\hline
\end{tabular}


Table S7 - Number of male forays, breeding stage and time of day. Table lists all factors included in a zeroinflated poisson generalized linear mixed model with log link function. The dependent variable was the number of forays undertaken per hour by a male ( $N=21,135$ observation hours, 24 males). Male ID was included as random factor (var $\pm s d: 2.8 \pm 1.67)$. Using backward elimination, the estimate and standard error of the last model in which a factor was included are given. The test statistic $\left(\chi^{2}\right)$, degrees of freedom ( $\left.d f\right)$ and significance ( $p$ value) given were determined using the likelihood-ratio test (LRT). The factors distance (average distance of a male's nest box to other nest boxes) and relative lay date of the male's brood were included in the last model, independent of significance.

\begin{tabular}{|c|c|c|c|c|c|c|}
\hline & & Estimate & se & $\chi^{2}$ & df & $P$ value \\
\hline Intercept & & 7.12 & 2.65 & - & - & - \\
\hline \multirow[t]{5}{*}{ Breeding stage } & & & & 318.39 & 4 & $<0.001$ \\
\hline & fertile & -0.02 & 0.04 & & & \\
\hline & chick rearing & 0.24 & 0.05 & & & \\
\hline & incubation & 0.20 & 0.03 & & & \\
\hline & prefertile & -0.25 & 0.03 & & & \\
\hline \multirow[t]{4}{*}{ Daytime } & & & & 41.02 & 3 & $<0.001$ \\
\hline & early morning & -0.22 & 0.04 & & & \\
\hline & evening & -0.01 & 0.03 & & & \\
\hline & morning & -0.02 & 0.02 & & & \\
\hline Distance & & -17.0 & 6.0 & - & - & - \\
\hline Relative lay date & & 0.01 & 0.08 & - & - & - \\
\hline
\end{tabular}

Table S8 - Duration of male forays, breeding stage and time of day. Table lists all factors included in a linear mixed model with log transformed duration of forays undertaken by a male $(N=14,419$ forays, 24 males $)$ as dependent variable. Male ID was included as random factor (var $\pm s d: 0.005 \pm 0.07$, residual variance $\pm s d: 0.15$ \pm 0.38 ). The test statistic $\left(\chi^{2}\right)$, degrees of freedom ( $\left.d f\right)$ and significance ( $p$ value) given were determined using the likelihood-ratio test (LRT). The factors distance (average distance of a male's nest box to other nest boxes) and relative lay date of the male's brood were included in the last model, independent of significance.

\begin{tabular}{|c|c|c|c|c|c|}
\hline & Estimate & se & $\chi^{2}$ & df & P value \\
\hline Intercept & 1.59 & 0.02 & - & - & - \\
\hline \multirow[t]{4}{*}{ Daytime } & & & 24.31 & 3 & $<0.001$ \\
\hline & 0.04 & 0.01 & & & \\
\hline & -0.02 & 0.01 & & & \\
\hline & -0.02 & 0.01 & & & \\
\hline Distance (scaled) & -0.02 & 0.02 & - & - & - \\
\hline Relative lay date (scaled) & -0.01 & 0.02 & - & - & - \\
\hline \multicolumn{6}{|l|}{ Dropped terms } \\
\hline Breeding stage & & & 2.83 & 4 & 0.59 \\
\hline
\end{tabular}


Table S9 - Number of nest box areas visited per day by females and breeding stage. Table lists all factors included in a poisson generalized linear mixed model with log link function. The dependent variable was the number of nest box areas visited per day by a female $(N=1584$ observation days, 26 females). Female ID was included as random factor (var $\pm s d: 1.0 \pm 1.0)$. Using backward elimination, the estimate and standard error of the last model in which a factor was included are given. The test statistic $\left(\chi^{2}\right)$, degrees of freedom (df) and significance ( $p$ value) given were determined using the likelihood-ratio test (LRT). The factors distance (average distance of a female's nest box to other nest boxes) and relative lay date of the female's brood were included in the last model, independent of significance.

\begin{tabular}{|c|c|c|c|c|c|c|}
\hline & & Estimate & se & $\chi^{2}$ & df & $P$ value \\
\hline Intercept & & -0.68 & 0.22 & - & - & - \\
\hline \multirow[t]{5}{*}{ Breeding stage } & & & & 180.39 & 4 & $<0.001$ \\
\hline & fertile & 0.38 & 0.11 & & & \\
\hline & nestlings & -0.26 & 0.14 & & & \\
\hline & incubation & -0.47 & 0.12 & & & \\
\hline & prefertile & 0.52 & 0.09 & & & \\
\hline Distance (scaled) & & -0.38 & 0.20 & - & - & - \\
\hline Relative lay date (scaled) & & 0.33 & 0.20 & - & - & - \\
\hline
\end{tabular}

Table S10 - Number of nest box areas visited per day by males and breeding stage. Table lists all factors included in a poisson generalized linear mixed model with log link function. The dependent variable was the number of nest box areas visited per day by a male $(N=1417$ observation days, 24 males). Male ID was included as random factor (var $\pm s d: 1.26 \pm 1.12$ ). Using backward elimination, the estimate and standard error of the last model in which a factor was included are given. The test statistic $\left(\chi^{2}\right)$, degrees of freedom ( $\left.d f\right)$ and significance $(p$ value) given were determined using the likelihood-ratio test (LRT). The factors distance (average distance of a female's nest box to other nest boxes) and relative lay date of the female's brood were included in the last model, independent of significance.

\begin{tabular}{|c|c|c|c|c|c|c|}
\hline & & Estimate & se & $\chi^{2}$ & df & P value \\
\hline Intercept & & -0.50 & 0.25 & - & - & - \\
\hline \multirow[t]{5}{*}{ Breeding stage } & & & & 17.0 & 4 & 0.002 \\
\hline & fertile & 0.19 & 0.10 & & & \\
\hline & nestlings & -0.12 & 0.13 & & & \\
\hline & incubation & 0.21 & 0.10 & & & \\
\hline & prefertile & 0.21 & 0.08 & & & \\
\hline Distance (scaled) & & -0.64 & 0.25 & - & - & - \\
\hline Relative lay date (scaled) & & 0.10 & 0.25 & - & - & - \\
\hline
\end{tabular}


Table S11 - Occurrence of extra-pair offspring and average number of male visits per hour. The table consists of all factors included in a binomial generalized linear model with occurrence of extra-pair offspring $(0 / 1)$ as the dependent variable $(N=37$ broods). Given is the estimate, test statistic ( $Z$ value) and significance ( $p$ value).

\begin{tabular}{lllll}
\hline & Estimate & se & Z value & P value \\
\hline Intercept & 1.72 & 2.24 & - & - \\
Average male visits & -0.23 & 0.36 & -0.71 & 0.48 \\
Distance & -3.34 & 4.46 & -0.75 & 0.45 \\
Relative lay date & -0.003 & 0.07 & -0.046 & 1.0 \\
\hline
\end{tabular}






\section{CHAPTER 3}

No evidence for eavesdropping on male singing interactions in the great tit (Parus major)

Nina Bircher, Kees van Oers, Marc Naguib

Submitted 


\section{Abstract}

Observing interactions between others can provide important information to individuals. Male songbirds often engage in song contests in which they signal more or less dominantly by choosing the type and timing of signals. Yet, how other individuals use such information is still poorly understood. Here we tested whether great tits (Parus major) use information gained by eavesdropping on male singing interactions to assess rivals and (potential) mates. We used interactive playback experiments to engage territorial males in song contests with either a more or less challenging intruder. We followed male and female movements before and after interactions by radio-tracking, determined paternity using microsatellite analysis and maternal investment by quantifying egg weights and provisioning behavior. We expected males facing a more challenging intruder to respond with stronger vocal and spatial responses. Moreover, we expected that these males would be perceived as "losing" the interaction and their mates to foray more often off-territory to assess potential extra-pair mates and invest less in their broods. Additionally, we expected neighboring females to visit territories of such males less often, but male neighbors to intrude more in these territories. We found that males exposed to playbacks did not respond differently to the two treatments and their mates did not alter their foraying behavior or brood investment. Moreover, both female and male neighbors did not change their visiting behavior to playback territories after either of the treatments. Our results thus provide no evidence that females use information gathered by eavesdropping on song interactions of their mate in reproductive decisions or that information gained by eavesdropping change closerange associations between individuals in a territorial neighborhood. Although eavesdropping can provide important information, reproductive decisions and conspecific assessment may be based on multiple sources of information gathered over time and not necessarily be modified by single interaction events. 


\section{Introduction}

For many animals, social information plays a central role in individual decision making and can influence the choice of, for example, foraging places, breeding sites, or mates. One way of gathering information about available options is through observing the outcome of interactions between others (Danchin et al., 2004). Aggressive interactions between males, for example, can provide information to uninvolved bystanders ("eavesdroppers") about motivation and fighting ability of both contesters and be used by these other individuals to adjust their own behavior (Doutrelant and McGregor, 2000; Mennill et al., 2002; Oliveira et al., 1998; Silk, 1999). In many territorial songbirds males engage in countersigning interactions and such vocal interactions are particularly well suited for eavesdropping, as acoustic signals range far and thus allow others to eavesdrop and gain information without risking close-range interactions (McGregor and Peake, 2000; Todt and Naguib, 2000). Several studies have shown that both male (Akçay et al., 2013; Naguib and Todt, 1997; Peake et al., 2001) and female (Amy et al., 2008; Mennill et al., 2002; Otter et al., 1999) songbirds eavesdrop on such interactions.

Counter singing males can alter their vocal responses by either changing the type of signal or the timing of signals. How male songbirds are using their signals in singing interactions might communicate their fighting ability or willingness to escalate an interaction (Todt and Naguib, 2000). Matching the signal type of the other individual is associated with escalated encounters and perceived as increased threat by males in many species (Akçay et al., 2013; Mennill \& Ratcliffe, 2004; Todt \& Naguib, 2000; Vehrencamp, 2001, but see for example Baker, Wilson, \& Mennill, 2012; Searcy, Anderson, \& Nowicki, 2006). Similarly, overlapping another's signal in time by starting a song before the opponent has finished singing has been shown to elicit strong responses and is often interpreted as signal indicating the willingness to escalate an interaction (Dabelsteen et al., 1996; Helfer and Osiejuk, 2015; Naguib and Mennill, 2010; Searcy and Beecher, 2009; Todt and Naguib, 2000). Previous studies have also shown that, just like overlapping and matching another male's song, singing on a intruded territory for longer elicits a stronger response in resident males (Poesel and Dabelsteen, 2005; Sprau et al., 2014). Eavesdropping on interactions between males and the singing behavior of intruders can thus inform other males about the level of threat posed by rivals (Mennill and Ratcliffe, 2004; Naguib et al., 2004; Peake et al., 2002). Aspects of male singing have on the other hand been shown to play an important role in female choice of both social and copulations partners in many bird species (Catchpole \& Slater, 2008; Searcy \& Yasukawa, 1996). Females might use the information gathered by eavesdropping in their sampling of potential (extra-pair) mates or 
to adjust their level of maternal investment. Females seem to show a preference for males that are overlapping their opponents frequently: Female domestic canaries (Serinus canaria) performed more copulation solicitation displays when exposed to song they had previously heard to be the overlapping song in an interaction (Amy et al., 2008; Leboucher and Pallot, 2004), and a radio-tracking study in great tits, following the movements of neighbors of playback subjects, showed that females were more attracted to territories of males that overlapped a simulated intruder often compared to males that did not (Snijders et al., 2017a).

However, only few studies have tested whether female birds actually use eavesdropping in reproductive decision making: Otter et al. (1999) engaged territorial male great tits in interactive playback experiments and subsequently observed female movements to territories of other males using focal follows. While they found that female great tits paired with a male that lost a contest, because its songs were overlapped more often, were more likely to foray in to territories of other males (Otter et al., 1999), these forays did not lead to a higher likelihood of having extra-pair offspring (Otter et al., 2001). In contrast, the mate choice decisions of female black capped chickadees (Poecile atricapillus) were associated with information gained through eavesdropping: High-ranking males that lost a song contest against a simulated intruder, because their song was overlapped and matched more often, had a higher proportion of extra-pair chicks in their brood than high ranking males that did not lose the interaction. Losing or winning an interaction did however not influence paternity in the broods of lowranking males (Mennill, Boag, \& Ratcliffe, 2003; Mennill et al., 2002). Female domestic canaries stimulated with song they previously heard as overlapping song in an interaction, laid eggs with a higher yolk content than females that were exposed to song that has previously been overlapped by another male. Other measures of resource investment such as egg mass and testosterone concentration in the yolk were however not affected by eavesdropping (GarciaFernandez et al., 2010). Thus, there is conflicting evidence about the extent to which females use eavesdropping as a strategy to assess potential (extra-pair)-mates and to differentially allocate resources to their brood. Moreover, most studies focused on the eavesdropping response of a resident male and/or its mate, but singing interactions can be heard across a larger neighborhood. Without considering how singing interactions affect movements and behavioral decisions by eavesdropping neighbors (Fitzsimmons et al., 2008a; Naguib et al., 2004; Snijders et al., 2017a) our understanding on their role in social and communication system thus remains incomplete. 
Here, we tested whether female and male great tits (Parus major) eavesdrop on male song interactions and alter their behavior based on the available information. We used interactive playback experiments to simulate territory intrusions just before and during egg laying, a key time period for female reproductive decision making. We simulated more challenging and persistent intruders by overlapping a male's song and singing on the territory a for longer time and less challenging intruders by alternate singing and singing for a shorter time. We used an automated radio-tracking system to track movements of both females and males into other territories ("forays"), determined paternity in broods of playback subjects and measured egg weights and provisioning effort as female investment in their broods. We tested whether 1) males respond with a stronger vocal response to more challenging intruders, 2) mates of males that faced a more challenging intruder (i.e. "lost" the interaction) foray more often to other territories and invest less in their broods, 3) female neighbors are less attracted to territories of males after they lost an interaction and male neighbors were more likely to foray into a territory of males after they lost an interaction. We expected that males would perceive an intruder that overlaps their song during an interaction and sings for longer on their territory as more threatening and thus respond with a stronger vocal response and approach the loudspeaker more closely. Moreover, we predicted that mates of such males would be more likely to foray offterritory to assess other males, lay lighter eggs and reduce their provisioning effort in response to the playback treatment since females would perceive the "loser" as less attractive. Because "losing" males should be perceived as less attractive, we predicted a priori that neighboring females would be less attracted to territories of these males. In contrast, we predicted that neighboring males would perceive "losing" males as less of a threat and be more likely to intrude into territories of these males. We subsequently also tested whether male and female neighbors change their overall foraying activity in response to playbacks. We expected that male neighbors would foray less after playbacks simulating a more challenging intruder, as they would be more prone to stay on their own territory to guard it. On the other hand, we expected female neighbors to increase overall foraying activity as they may be incited to assess intruders in the surrounding area. 


\section{Materials and methods}

\section{Study population and general field methods}

We conducted this study in a long-term study population of great tits in Westerheide, near Arnhem, The Netherlands. Westerheide is a forest of mixed wood with approximately 200 nest boxes distributed over a 1000 x 1200 m area. From mid-March until mid-June we routinely checked nest boxes to determine the start of nest building, egg laying, hatching and fledging. In the end of March, prior to the start of nest building, we tagged 84 birds with an Encounternet tag and we carried out playback experiments just before and during egg laying (see below). We caught parents when the chicks were 10 days old (day 0 being the hatching date) using spring traps and equipped them with an RFID tag embedded in a leg ring (Eccel Technology LTD, Glenfield, UK). We removed all remaining radio-tags (Encounternet) from birds at that point. We measured tarsus length and wing length (as length of the third primary) to the nearest $\mathrm{mm}$ and weight. We ringed chicks with an individual aluminium ring when they were 14 days old and measured tarsus length and weight. For paternity analysis, we collected two blood samples of approximately $10 \mu \mathrm{l}$ from the brachial vein of each parent and one of each chick. We suspended collected blood samples in Eppendorf tubes containing $1 \mathrm{ml}$ of Queen's lysis or Cell lysis buffer.

\section{Playback stimuli}

To construct the songs used as playback stimuli we used songs of male great tits recorded at least eight years previous to the experiment at our study site. It thus was unlikely that individuals exposed to the playbacks had heard the exact same songs before. All stimuli songs were recorded using a Sennheiser ME66/K6 or ME67/K6 microphone (Sennheiser Electronic GmbH \& Co. KG, Wedemark, Germany) connected to a Marantz PMD660 recorder (D\&M Holdings Inc., Kanagawa, Japan) with a sample frequency of $44.1 \mathrm{kHz}$ and resolution of 16 bit. We constructed 44 stimuli songs with Avisoft SASLAB PRO (Raimund Specht, Berlin, Germany) with songs from 44 different males following the procedure in Amy, Sprau, De Goede, \& Naguib (2010): songs were filtered with a $2000 \mathrm{~Hz}$ high-pass filter and adjusted to the same peak amplitude. We then constructed songs that each consisted of six identical syllables with two or three elements at a syllable rate natural for the respective song type. Stimulus songs used in playbacks and included in analyses $(N=33)$ had a duration of $2.5 \pm$ 0.09 s (mean \pm s.e.). 
Playback protocol and male vocal response measures

We carried out 44 playback experiments in total, but excluded 11 playbacks from all analyses as they were conducted when the female was already incubating $(N=5)$, the nests were abandoned during egg laying $(N=2)$, or the recordings were not of sufficient quality to measure the vocal response variables and verify that the treatment was successful $(N=4)$. Out of the remaining 33 playbacks, most $(N=29)$ were carried out with the social mate of radio-tagged females. For the analysis of neighbor responses to playback we also included playbacks conducted at boxes of females without a radio-tag $(N=4)$. All playbacks were conducted between 0700 and 1400 hours, except one that took place at 1600 hours. We carried out playbacks close to the nest box of the female just before or during egg laying (on average on day four of egg laying). We determined the nest box of radio-tagged females during regular tracking rounds in the evening to detect females when roosting. We used two Megaboom loudspeakers (Ultimate Ears, Newark, CA, USA) placed within maximal 20m of each other and the nest box. Distances were measured with a Leica Rangemaster CRF 900 (Leica Geosystems AG, Heerbrugg, Switzerland). Playbacks consisted of a lure phase and an interactive phase. We played the lure song from one of the two loudspeakers and subsequently the interactive treatment from the other loudspeaker in order to standardize the distance between the subject and the interactive loudspeaker. All songs were played at a sound pressure level of $84-86 \mathrm{~dB}$ SPL at $1 \mathrm{~m}$. This broadcast level was determined beforehand using the playback equipment and a Voltcraft Plus digital sound-level meter 200 with A weighting and fast response at a location outside of the study site. We measured the SPL at $1 \mathrm{~m}$ for both a sine tone $(2 \mathrm{kHz})$ and an example great tit song normalized to the same peak amplitude as the playback songs. Our broadcast level was thus similar to the estimated natural SPL at $1 \mathrm{~m}$ for great tit song (Blumenrath and Dabelsteen, 2004). We used the same lure song for all subjects. For the interactive part of the playback, we randomly assigned a different stimulus song to each bird. We initiated each playback when the female was present in the vicinity of the nest box (determined by radio tracking for tagged females) by playing the non-interactive lure song on a loop for a maximum of $2 \mathrm{~min}$ to alert the social mate and incite it to sing. When the male started to sing, we stopped the lure song and started the interactive part of the playback with one of the two treatments (overlapping or alternating). If the male did not respond with song after 2 min of lure, we aborted the experiment and tried again the next day.

In the alternating treatment $(N=15)$, we waited with broadcasting a song until the male had finished a song and we stopped the playback if the male ceased to sing completely. In the 
overlapping treatment $(N=18)$, we broadcast a song as soon as the male started a song and we continued broadcasting songs at regular intervals (up to a maximum of 36 songs) even if the male ceased to sing completely. We played a maximum of 22 songs (range 4 - 22) in the alternating and 36 (range 26 - 36) songs in the overlapping treatment. In the overlapping treatment, playbacks lasted significantly longer (unpaired t-test: $T=-3.1, P=0.005, d f=31$ ) and songs of subjects were overlapped more often (Mann-Whitney rank-sum test: $W=0, P<=$ 0.001). Playbacks lasted for $4.2 \pm 0.25$ min (mean \pm s.e.) in the overlapping treatment and 2.9 \pm 0.36 min (mean \pm s.e.) in the alternating treatment. We overlapped $61.4 \pm 4 \%$ (mean \pm s.e.) of the songs sung by a subject in the overlapping and $6.6 \pm 1.8 \%$ (mean \pm s.e.) in the alternating treatment, which is similar to the rate used in a previous study (Amy et al., 2010).

We recorded the songs of the focal male during the interactive playback with a Sennheiser M66/K6 microphone on to one channel with a Marantz PMD660 recorder (sample frequency $44.1 \mathrm{kHz}$; resolution $16 \mathrm{bit}$ ). On the other channel, we recorded spoken notes with a second microphone. From the recordings made during and after the playback we measured for each male 1) the song rate during the interaction (number of songs per minute) 2) the mean duration of songs during the interaction 3 ) the mean proportion of songs overlapped by the simulated intruder and 4) the number of songs within 1 min after the treatment ended. All analyses were done in Avisoft SASLAB PRO. We also noted the closest approach to the treatment loudspeaker for each male $(0 \mathrm{~m},<10$, or $>10 \mathrm{~m})$ measuring the distance between loudspeaker and the closest song post with a Leica Rangemaster CRF 900 or with a measuring tape. We only tested males on the same day that were several territories apart.

\section{Radio tracking and spatial response measures}

We used the automated radio-tracking system "Encounternet" to follow the movements of birds (Mennill et al., 2012b; Snijders et al., 2017a, 2014). Encounternet consists of tags sending out digital individual ID signals every 5 seconds and receivers storing all signals of tags within range together with a signal strength measure (RSSI) and a time stamp. We caught female and male great tits during a routine roost check on the $22^{\text {nd }}$ of March 2017 and fitted them with an Encounternet tag of approximately $1.2 \mathrm{~g}$. From the end of March onwards we regularly checked nest boxes and mounted Encounternet receivers in trees above nest boxes when nest building activity started. We used the RSSI signal strength measure to estimate the distance between a bird and a receiver. We evaluated all movements as extra-territorial forays when birds approached a nest box other than their own within $15 \mathrm{~m}$ and remained within that range for at 
least $10 \mathrm{~s}$. This way we excluded cases where birds moved past another nest box without really staying there. We ran the Encounternet system from the $28^{\text {th }}$ of March to the $18^{\text {th }}$ of May. We did not have tracking data available for two of the females involved in playbacks, due to a technical problem with their radio-tags. For all radio-tagged females involved in playbacks with tracking data available $(N=27)$ we measured the number of forays made during the day before and after playback. When a female was successfully tracked, but no foray was recorded on the respective days, we added a zero to the dataset. We did not analyze the spatial response to playbacks of the playback subjects themselves as only eight of them were radio-tagged.

For the analysis of the neighbor spatial response we included all individuals that were likely to hear the playback interaction at the respective box. The range over which a signal can be detected and recognized by conspecifics varies depending on the signal characteristics, level of background noise, transmission characteristics of the environment and the sensitivity of the receiver (Brumm and Naguib, 2009). Great tit song has been estimated to range as far as $\sim 180$ m (Blumenrath and Dabelsteen, 2004). We thus included all neighbors within $150 \mathrm{~m}$ of the boxes exposed to playback in the neighbor response analysis. We excluded playbacks at five nest boxes from the analysis as they did not have any radio-tagged neighbors within $150 \mathrm{~m}$ or those neighbors never visited the area of the playback even during the entire tracking season. All remaining boxes $(N=26$, of which 14 received an overlapping and 12 an alternating treatment) had at least one (and maximum five) radio-tagged neighbors within $150 \mathrm{~m}$. In total 36 individuals $\left(N_{\text {females }}=23, N_{\text {males }}=13\right)$ were included as neighbors. We measured the number of visits by female and male neighbors to playback territories and the number of female and male neighbors visiting during the day before and after playback for each box. When no visits were recorded during the respective days by females and/or males, but the target nest box in the respective territory did have female and/or male neighbors that visited at other times, we added a zero to the dataset accordingly. Subsequently, we also analyzed the overall foraying behavior of close neighbors before and after playbacks, including their forays to all monitored territories $(N=74)$ within the study site, not only the territories within which playbacks took place. Some individuals $(N=6)$ were neighbors to more than one playback territory and the treatment days of these playbacks overlapped, thus we excluded these individuals from this analysis. We had data on overall foraying behavior for a total of 31 neighbors $(N$ females $=22, N$ males $=9)$ of 25 playback boxes $\left(N_{\text {overlapping }}=15, N_{\text {alternating }}=10\right)$. We measured the number of forays these individuals made on the day before and after playbacks took place in playback 
territories they were neighbors to. If individuals were successfully tracked during the entire time, but no forays were recorded on these days, we added a zero to the dataset accordingly.

\section{Egg weights and female provisioning behavior}

For 23 out of 33 nest boxes in the playback territories, we measured the weight of eggs laid before and after playback to the nearest centigram. Between day 12 and 14 after hatching we recorded female provisioning behavior continuously during an entire day using RFID readers (Dorset ID, Aalten, The Netherlands) with antennas mounted around the nest box opening. We obtained data on provisioning behavior for 29 of 33 females involved in a playback. Females might adjust the provisioning frequency and/or the duration of provisioning, thus we quantified both the time between the first and the last presumed provisioning visit ("working day length") and the provisioning rate (number of feeding visits/working day length in hours) for each female. Females sleep in the nest boxes overnight, thus we excluded the very first log of the day, as this was likely the time the female emerged from the box, and treated the second $\log$ as the first provisioning visit. We treated transponder reads within $17 \mathrm{~s}$ of each other as one visit, as they were likely caused by the female staying in the nest box or near the nest box opening when feeding as a comparison of RFID data and video recordings has shown (L. Zandberg personal communication).

\section{Parentage analysis}

We used five microsatellite markers to determine parentage: PmaTAGAn71, PmaGAn27-PET, PmaTGAn33-VIC, PmaC25-NED and PmaD105-FAM (Saladin et al., 2003). We extracted DNA from blood samples with the FavorPrep 96-well Genomic DNA Kit (Favorgen Biotech Corporation, Ping-Tung, Taiwan) and amplified the isolated DNA with the QIAGEN multiplex PCR kit (QIAGEN GmbH, Hilden, Germany) following the manufacturer's protocol. We prepared PCR products on ABI plates with a molecular size standard (GeneScan LIZ, Applied Biosystems, Foster City, CA, USA). The sequence analysis of PCR products was carried out by BaseClear BV (BaseClear BV, Leiden, The Netherlands). We determined the size of the sequenced PCR products and derived the genotype for each individual using in GeneMapper v. 5.0 (Applied Biosystems). We determined whether a chick was within-pair or extra-pair with CERVUS v. 3.0.7 (Kalinowski et al., 2007; Marshall et al., 1998) testing all chicks against their putative fathers using the following parameters: $98 \%$ of loci typed, error rate $0.01 \%, 10$ '000 cycles and two candidate parents. We treated chicks as extra-pair if there were two or more mismatches with the putative father and the putative father was not the most likely parent 
according to the analysis in CERVUS. The combined exclusion probability for all microsatellites was $>99.9 \%$. One of our loci deviated significantly from the Hardy-Weinberg equilibrium when the genotypes of all sampled individuals in 2017 of the study population were included in the analysis (PmaTAGAn71: $\left.\chi^{2}=43.68, P<=0.001, d f=10\right)$, most likely due to the family structure of the data. We determined paternity in 29 out of the 33 playback boxes that were included in the analysis, of which 11 had EPO. We were unable to determine paternity in the remaining 4 boxes, because we did not catch the father to obtain a blood sample $(N=2)$, obtained genotypes of chicks were not matching the genotype of the mother $(N=1)$ or we did not have blood samples available for chicks as they died before the age of 2 weeks $(N=1)$.

\section{Statistical analysis}

Sample sizes vary between analyses because we were not able to collect all data for all nest boxes or individuals. We conducted all statistical analysis in R (R Core Team, 2019) and fitted all generalized linear (mixed) models and linear mixed models using the packages lme4 (Bates et al., 2015) and MASS (Venables and Ripley, 2002). To test whether treatments affected measures of male vocal response and female provisioning behavior (working day length and provisioning rate), we used unpaired T-tests and Mann-Whitney rank-sum tests. We tested whether egg weight was affected by treatments using a linear mixed model with time of laying (before or after playback) and treatment and their interaction (laying time*treatment) as fixed effects and nest box ID as random factor, and determined significance using likelihood-ratio tests (LRT). To compare female foraying behavior and neighbor visiting behavior between the day before and the day after the treatments we were not able to use Mann-Whitney rank-sum tests, as there were many cases in which both counts were zero and the test could not compute an exact p-value. We thus used poisson generalized linear models to model counts of forays and number of boxes visited on these days. In case of over dispersed counts we used quasipoisson generalized linear models. To test whether the number of forays females made and the number of boxes females visited (response variables) differed between the day before and after a playback we fitted a poisson or quasipoisson generalized linear model with the day relative to the day of playback (-1 and 1) as fixed effect. We fitted a separate model for females exposed to an overlapping and alternating treatment. We tested whether females with extra-pair offspring (EPO) differed from females without EPO in their response to treatments by fitting a poisson or quasipoisson generalized linear model with presence of EPO (yes/no) and treatment and their interaction (epo*treatment) as fixed effects and the number of forays made and number of boxes visited the day after the playback day as response variables. To test whether 
the number of visits by neighbors and the number of neighbors visiting a playback nest box (response variables) differed between the day before and after a playback we fitted a poisson or quasipoisson generalized linear model with the day relative to the day of playback ( -1 and 1) as fixed effect. We tested male and female neighbors separately for each treatment. Additionally, we tested whether the number of total forays by female neighbors (including forays to nest box areas other than playback boxes) differed between the day before and after playbacks. We fitted a quasipoisson generalized linear (mixed) model for each treatment separately with the day relative to the day of playback (-1 and 1) as fixed effect, adding female ID as random factor when some individuals in the respective data subset were neighbors to several boxes.

\section{Ethical note}

Birds involved in playback experiments were tagged as part of a larger study on foraying behavior from 2016-2017 and the permission for this work was granted by the Dutch legal entity Dier Experimenten Comissie (DEC) no. NIOO-10.05 to $\mathrm{MN}$ and $\mathrm{KvO}$ and no. NIOO 12.02 to $\mathrm{KvO}$. The Encounternet tags we used here were designed to be as small and light weight as possible, while still providing automated simultaneous tracking of multiple individuals. The weight of all tags deployed in 2016 and $2017(1.26 \pm 0.05 \mathrm{~g})$ was within the natural range of daily body weight changes of great tits (Van Balen, 1967). We tagged birds early in the breeding season before the start of egg laying. Birds were removed from roosting boxes during a routine roost check and brought to a car to fit tags using nylon leg-looped backpack harnesses. The entire procedure takes only a few minutes and afterwards birds were immediately brought back to the box they were caught in. We removed any remaining tags during standard capture sessions at the end of the season (when chicks were 10 days of age), up to two months after tagging. An analysis of possible tagging effects using the same type of tags (among others) in the same study population showed that tag effects depend on the timing of tagging (season start vs. during chick feeding) and the general environmental conditions of the breeding season: Under normal conditions at our study site and if fitted on birds early in the season as we did in 2016 and 2017, these tags were not found to have a negative impact on the likelihood of a bird breeding, provisioning behavior of parents, and condition of chicks raised by tagged parents (Snijders et al., 2017b). 


\section{Results}

\section{Effects of playback treatment on male vocal response}

Territorial males exposed to the overlapping treatment $(N=18)$ did not differ from males exposed to the alternating treatment $(N=15)$ in their song rate (unpaired t-test: $T=1.16, P=$ $0.26, d f=31$ ), mean song duration (Mann-Whitney rank-sum test: $W=161.5, P=0.35$ ), number of songs during the minute after playback (Mann-Whitney rank-sum test: $W=139, P=0.89$, Figure 1) and closest approach to the loudspeaker (Fisher's Exact Test, $P=0.26$ ).
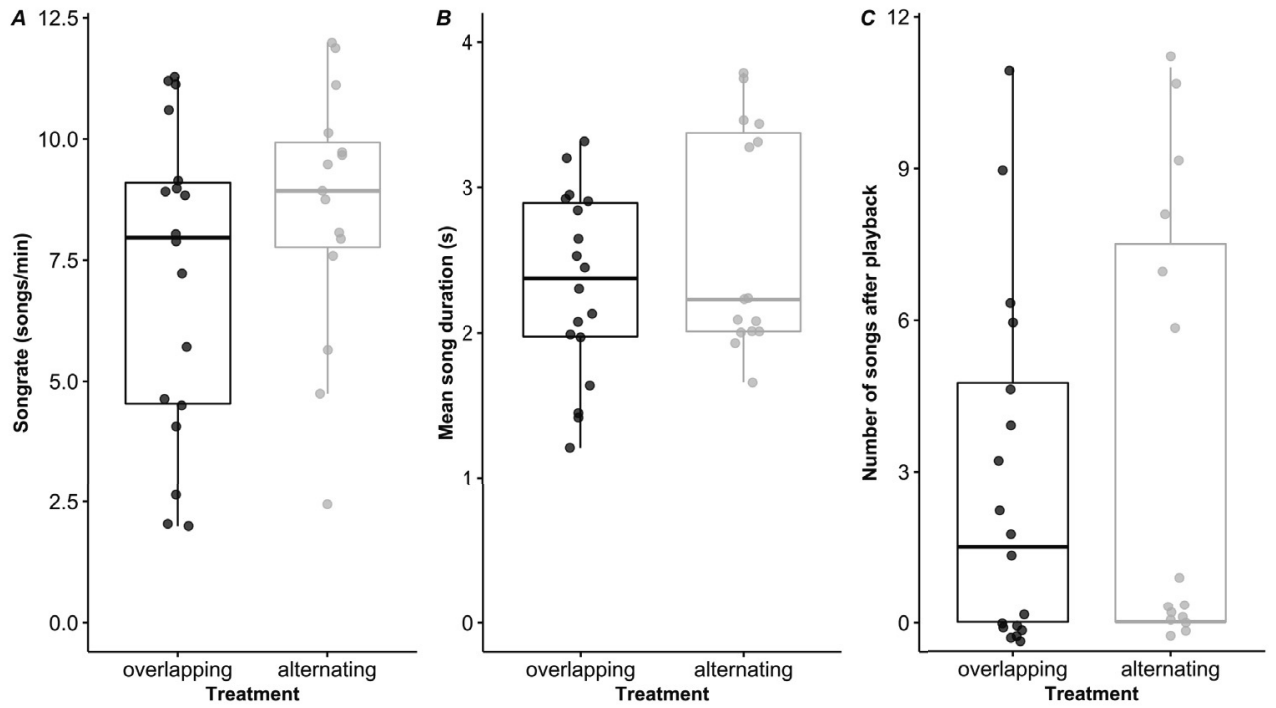

Figure 1 - Male vocal response. Males exposed to an overlapping treatment ( $N=18$; in black) did not differ from males exposed to an alternating treatment $(N=15$; in grey) in their song rate during the simulated interaction (number of songs per minute - Figure $1 \mathrm{~A}$ ), the mean duration of the songs sung during the interaction (in seconds - Figure 1 B) and the number of songs they sung during the minute after treatment end (Figure $1 C$ ).

\section{Effects of playback treatment on female spatial behavior}

Foraying behavior of radio-tagged females with social partners exposed to a playback was not affected by either the overlapping or alternating treatment: Females did not differ in the number of forays made or in the number of other territories visited between the day before and after the playback (female forays overlapping treatment: GLM: $T=-0.11, P=0.91, d f=29, N=15$, female forays alternating treatment: GLM: $T=-0.23, P=0.82, d f=23, N=12$, territories visited by females overlapping treatment: GLM: $T=0.60, P=0.56, d f=29, N=15$, territories 
visited by females overlapping treatment: GLM: $Z=1.26, P=0.21, d f=23, N=12$, Figure 2 ). Females with extra-pair offspring (EPO) $(N=9)$ did not differ in their response to the playback treatments from females without EPO $(N=15)$ (number of forays on day after playback: epo*treatment: GLM: $T=0.51, P=0.61, d f=23$, number of other boxes visited on day after playback: epo*treatment: GLM: $Z=0.20, P=0.85, d f=23)$.

Effects of playback treatment on egg weights and female provisioning behavior

Eggs laid before and after an alternating treatment or before and after an overlapping treatment did not differ in their weight (LMM: $\chi^{2}{ }_{3}=1.35, P=0.72, N=184$ eggs of 23 females). Females with social mates exposed to overlapping treatments did not differ from females with social mates exposed to alternating treatments in their working day length, i.e. the duration between the first and the last nest box visit, (Mann-Whitney rank-sum test: $W=77, P=0.25, N=29$ ) and provisioning rate (unpaired t-test: $T=0.81, P=0.46, d f=27, N=29$ ).
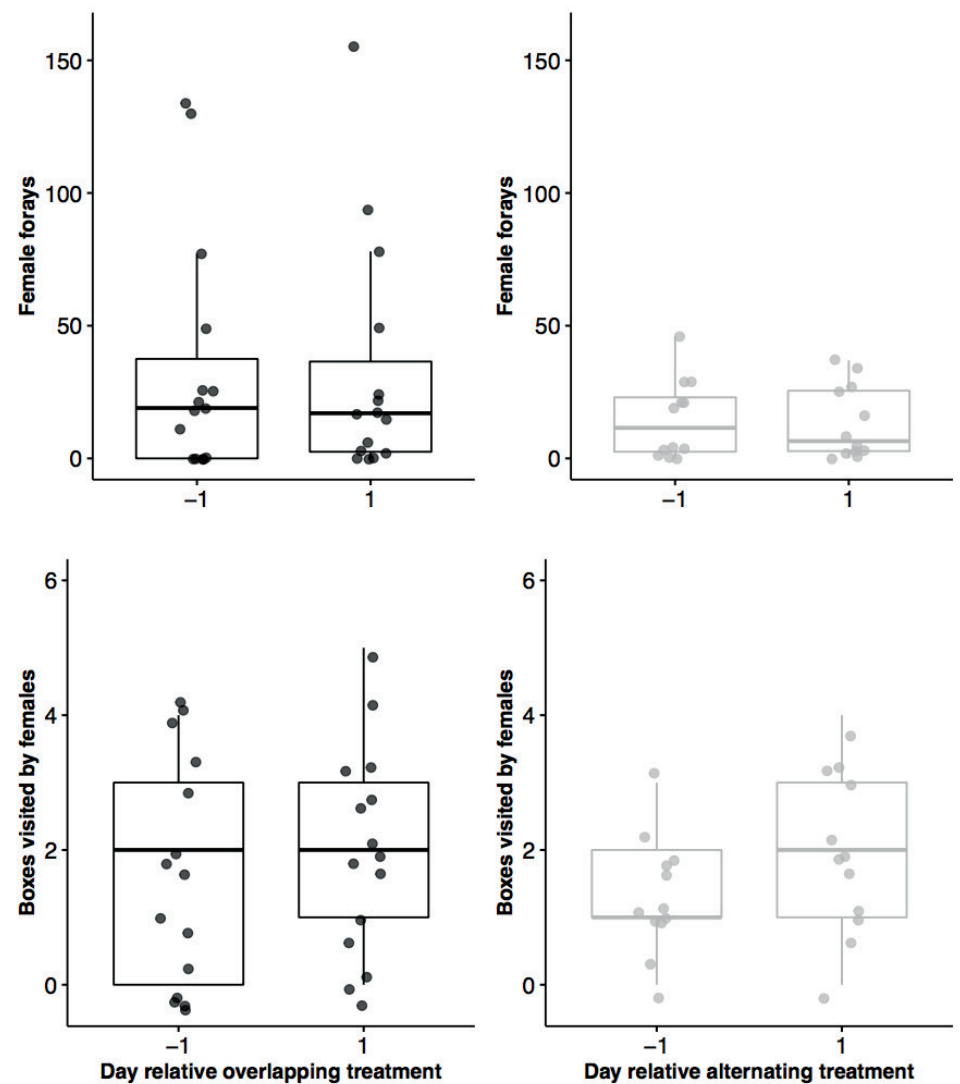
Figure 2 - Spatial response of females. Female foraying behavior (number of forays and number of territories visited) did not differ between the day before (day -1) and the day after (day 1) the playback for either the overlapping $\left(N_{\text {females }}=15\right.$; in black $)$ or the alternating treatment $\left(N_{\text {females }}=12\right.$; in grey).

\section{Effects of playback treatment on neighbor spatial behavior}

Visiting behavior to playback nest box areas of both female and male neighbors did not differ between the day before and day after for either treatment (female visits overlapping treatment: GLM: $T=0.23, P=0.82, d f=23, N=12$, female visitors overlapping treatment: GLM: $T=-$ $1.20, P=0.24, d f=23, N=12$, male visits overlapping treatment: GLM: $T=-0.62, P=0.54$, $d f=17, N=9$, male visitors overlapping treatment: GLM: $Z=-0,47, P=0.64, d f=17, N=9$, female visits alternating treatment: GLM: $Z=0.30, P=0.76, d f=21, N=11$, female visitors alternating treatment: GLM: $T=0.23, P=0.82, d f=21, N=11$, male visits alternating treatment: GLM: $T=0.06, P=0.97, d f=11, N=6$, male visitors alternating treatment: GLM: $Z=-0.57, P=0.57, d f=11, N=6)$. Moreover, female neighbors did not change their overall foraying activity (overlapping treatment: GLMM: $T=0.37, P=0.72, d f=23, N_{\text {females }}=16$, alternating treatment: GLM: $Z=-0.35, P=0.73, d f=21, N_{\text {females }}=11$, Figure 3$)$. We were not able to test overall foraying activity of male neighbors due to the small sample size $\left(N_{\text {males }}=\right.$ 9). 

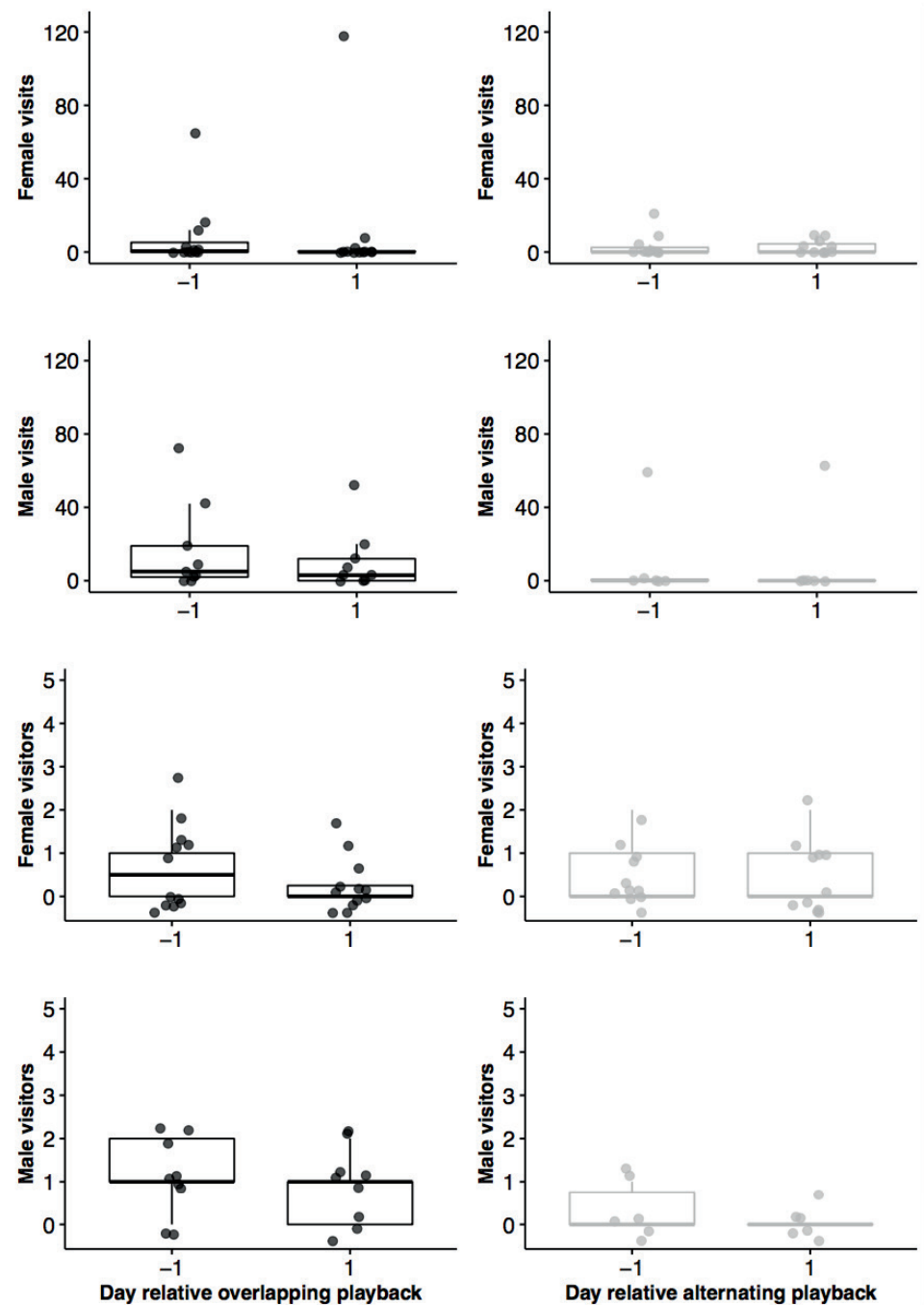

Figure 3 - Visiting behavior of close neighbors to playback territories. Neither the overlapping (in black) nor the alternating (in grey) treatment affected female or male neighbor visits to territories where playbacks took place ( $N_{\text {overlapping boxes with female neighbors }}=12, N_{\text {overlapping boxes with male neighbors }}=9, N_{\text {alternating boxes with female neighbors }}=11, N$ alternating boxes with male neighbors $=6$ ).

\section{Discussion}

Here we show that territorial male great tits did not react with a stronger vocal or spatial response to a more overlapping and persistent intruder than to a less persistent intruder that alternated songs. Moreover, whether or not intruders were overlapping and persistent did not influence the behavior of females these males were paired with: Females neither changed their foraying behavior nor their investment in broods (weight of eggs and provisioning of chicks) 
after playbacks and females having extra-pair offspring did not foray more often to other territories in response to intrusions than females without extra-pair offspring. Neither female nor male neighbors changed their spatial behavior in response to the simulated intrusions.

A vast number of studies showed that male songbirds respond differently in an overlapping versus a non-overlapping context, usually responding with a stronger vocal response and closer approach to an overlapping simulated rival (Helfer and Osiejuk, 2015; Naguib and Mennill, 2010). As a consequence, song overlapping is often interpreted as signal of threat as it leads to increased vocal and spatial responses (Naguib and Mennill, 2010; Searcy and Beecher, 2009). Males in our study did not respond more strongly in their singing or approach when confronted with a simulated intruder that overlaps more often. This suggests that overlapping, even in combination with an intruder being very persistent by interacting for longer, was not perceived as more threatening. The level of overlapping in our experiment $(61.4 \pm 4 \%)$ was only slightly lower than in a previous study in the same population that found a strong male response (Amy et al., 2010; 68\%) and we here even combined the overlapping with the intruder being more persistent, which has been shown to elicit stronger responses as well (Poesel and Dabelsteen, 2005; Sprau et al., 2014). Nevertheless, we cannot rule out that the intruder in our study was perceived as less challenging by the resident males than in previous studies as many studies reporting a stronger response used higher levels of overlapping (e.g. $86-100 \%$ in great tits; Dabelsteen et al., 1996; Peake et al., 2002). A study in nightingales (Luscinia megarhynchus) using several different levels of overlapping during nocturnal song showed that higher levels of overlapping elicit stronger vocal responses (Naguib and Kipper, 2006). This indeed indicates that overlapping might be a graded signal with increasing levels signaling an increasing willingness to escalate an interaction. Yet, in nightingales vocal responses of subjects during nocturnal singing were already observed at moderate levels of overlapping of $25 \%$ (Naguib and Kipper, 2006), which is much lower than the level of overlapping we used in this study. Moreover, the few studies that have investigated overlapping levels in naturally occurring song contests, that were not playback induced, (Fitzsimmons et al., 2008b; Foote et al., 2010) report relatively low levels of overlapping of 16 to $35 \%$ (Fitzsimmons et al., 2008b; Foote et al., 2008a; Naguib and Kipper, 2006). However, interactions between neighboring males considered in these studies might not reflect singing behavior during territorial close-range conflicts with unknown intruders. Future studies investigating the overlapping level in natural interactions during territorial conflicts would thus be helpful do discern whether the slightly 
lower level of overlapping in our study could have resulted in similar responses to both playback treatments.

We did not find any evidence that females paired to males involved in playbacks used information gathered by eavesdropping in reproductive decision making: Females neither changed their foraying behavior to other territories nor their investment in broods. Previous studies suggested that females use information gained by eavesdropping to assess the quality of males relative to others and accordingly alter reproductive decisions (Garcia-Fernandez et al., 2010; Mennill et al., 2002; Otter et al., 1999). Otter et al. (1999) found that female great tits that were paired with a male that "lost" its interaction to an overlapping intruder forayed offterritory significantly more often and preferably into territories of males that had "won" their interaction, presumably to seek extra-pair copulations with better quality males (Otter et al., 1999). However, subsequent paternity analyses did not reveal that these females were more likely to have extra-pair offspring (Otter et al., 2001). In contrast, in our study we did not observe that females forayed more often after their social mate was exposed to overlapping playback. Moreover, females with extra-pair offspring (EPO) did not differ in their foraying behavior after either of the treatments from females without EPO. Based on the study by Mennill et al. (2002), we had expected females with EPO to more readily reassess potential extra-pair partners after their social mate lost an interaction. Since we established that females were present during the entire treatment via radio-tracking for radio-tagged females and via visual observations for the four untagged females in our study, it is unlikely that the lack of female response is due to them not having been able to eavesdrop on the interaction. Females here thus did not appear to use eavesdropping to (re-)assess (potential) mates, but rather appear to have used the response by their mate as yard stick, and thus, like their mate, did not respond differently to the two playback treatments. Females probably have long-term experience with their mate and information on many interactions, and thus may not re-assesses reproductive decisions based on a single interaction. Re-assessment based on a single interaction, as reported for black capped chickadees (Mennill et al., 2002), may be due to an exceptionally strong change in perceived quality of the mate: only females mated with dominant black-capped chickadees that lost their interaction were more likely to adopt a mixed mating strategy, whereas females mated with subordinate males losing an interaction did not change their mating decisions, possibly because their mate loses more interactions in general and thus did not suffer as big of a change in perceived quality. Another reason that we did not observe a change in female foraying behavior could be that at the time of our playbacks (mostly during early egg 
laying) males increase mate guarding effort, maybe even more so after the simulated intrusion, and thus prevented females from foraying. We were not able to test this, as most of the playback subjects were not radio-tagged. In several other species however, the first eggs of a clutch are more likely to be fertilized by extra-pair mates than eggs laid later (Cordero et al., 1999; Krist et al., 2005; Schlicht et al., 2012). Thus, the time window in which females are most likely to seek extra-pair copulations might have been prior to playback.

We found no evidence that females allocate resources differentially based on information gathered by eavesdropping. Previous studies have shown that female allocate androgens (mostly testosterone) differentially to eggs based on male quality signaled by song (Gil et al., 2004, 1999a; Tanvez et al., 2004). Garcia-Fernandez et al. (2010) showed that female canaries laid eggs with greater yolk to egg ratio when exposed to song they previously had heard as being the overlapping song in a song contest, while other measures of resource investment, including egg weight, were not affected by being exposed to overlapping song (GarciaFernandez et al., 2010). We did not determine yolk to egg ratio or testosterone concentrations for our study and it is possible that females did in fact alter their investment, but we did not detect it with our measurements of egg mass and provisioning behavior. However, females have been shown to change investment in both egg mass (Cunningham and Russell, 2000) and chick provisioning (Limbourg et al., 2004), in studies that did not use song characteristics to signal male attractiveness. Similarly, as for female foraying, it might thus be possible that females would use information from song contests in differential resource allocation, but that this single playback experiment was not strong enough to actually change the perceived quality of their social mate which may be based on more information gathered over a longer time period.

Intrusions into one territory can be considered as a threat to the whole established neighborhood, thus neighbors are expected to monitor the situation around them (Fitzsimmons et al., 2008a; Naguib et al., 2011; Peake et al., 2002; Snijders et al., 2017a). The nature of the intrusions and song contests then could also lead to more territory prospections by other males and visits by females depending on how they perceive the intruder and the response by the resident. Contrary to our expectations, male and female neighbors did not change their spatial behavior in response to treatments, neither the more challenging nor the less challenging one. In our study, neighbors could have assessed both the relative differences between the intruder and the resident male and the singing behavior of the intruder alone. Previous studies showed that both female and male neighbors attend to relative differences of two contesters in an 
interaction and changed their spatial behavior in response (Otter et al., 1999; Snijders et al., 2017a). In contrast, neighbors here did not change their visiting behavior to intruded territories after the playbacks, suggesting that they did not perceive the resident males that faced these intrusions any differently than before. Similar as the mates of playback subjects, neighbors might not have perceived the relative differences between the intruder and the resident male as strong enough. Interestingly, neighbors also seemed not alarmed by the singing of the intruder alone. The simulated intruder was singing for longer and at higher rate in the overlapping treatment than in the alternating one and a high singing activity is often associated with social status (Otter et al., 1997) and can predict aggressiveness (Baker et al., 2012). Even if not attending to the relative differences between the interacting individuals, male neighbors could have been more alarmed by the presence of such an intruder and thus have visited those areas less often, while female neighbors might have been incited by hearing dominant intruders to move to such areas more and assess the males. However, while the intruder' singing behavior differed between the two treatments, the singing behavior of the resident males did not and neighbors might have been reassured by the lack of a strong vocal response of their established neighbor. Although neighboring males did not change their foraying behavior to the intruded territory specifically, they might still have been alarmed by the presence of the simulated intruders and more prone to guard their own territory and thus foray less over all. We were not able to test this due to the low number of male neighbors with known overall foraying activity before and after playbacks. Moreover, it is possible that neighbors reacted in ways we cannot assess here: neighboring males may not respond spatially, but react vocally through a higher song output as was observed in black-capped chickadees (Fitzsimmons et al., 2008a) and nightingales (Naguib et al., 2004). Female neighbors did not change their overall foraying activity, suggesting that such a short one-time intrusion event did neither alarm them nor incite them to move around to assess singing males, possibly because they would need more information on identity and location of the intruding individual.

\section{Conclusion}

Our results provide no support that song overlapping and singing for a longer time during a territory intrusion is perceived as a higher threat by territorial males, as males exposed to playbacks did not react with a stronger vocal or spatial response to overlapping intruders. Moreover, we found no evidence that females use information gained by eavesdropping on male song contests for reproductive decision making. The lack of the mates' spatial and reproductive responses suggests that, even if overlapping and singing for longer were perceived 
as more threatening, information gained by eavesdropping on a single interaction may not overrule previous assessments of mate quality or females may only act based on such information during limited time windows. Additionally, neighboring males and females did not change their prospecting behavior to playback territories or females their overall foraying activity in response to simulated intrusions, indicating that eavesdropping on male interactions may not necessarily influence close-range associations between individuals and overall alertness in a territorial neighborhood. Overall, while a growing body of studies clearly shows the importance of eavesdropping on male song contests for information gathering, our study emphasizes that further investigation is needed to pinpoint which aspects of male singing in interactions specifically are assessed and how this information is used by others.

\section{Acknowledgements}

We would like to thank Geldersch Landschap \& Kasteleen that we were allowed to work in Westerheide and Rahman Rasyidi, Lisanne Meulendijks, Bas van Boomgaard, Sander Bik and Beril Yildiz for their help with data collection. We would also like to thank Piet de Goede, Christa Mateman, Martijn van der Sluijs, Lydia Nieuwe Weme and all the animal care takers at NIOO-KNAW for their help throughout the entire project. We are grateful to John Burt and Hans Meier for the technical support with the Encounternet system. We would also like to thank Camilla Hinde for her helpful comments on the measures of female investment and Lies Zandberg for her advice on the analysis of the RFID data. This study was permitted by the Dutch legal entity Dier Experimenten Comissie (DEC) no. NIOO-10.05 to $\mathrm{MN}$ and KvO and no. NIOO 12.02 to KvO. The project was funded by an NWO-ALW open competition grant (824.15.012) to $\mathrm{MN}$. 



\section{CHAPTER 4}

Does singing behavior during song contests reflect relative differences between males in the great tit (Parus major)?

Nina Bircher, Kees van Oers, Daniel J. Mennill, Bernice Goffin, Marc Naguib In preparation 


\section{Abstract}

Eavesdropping on interactions between others provides a low-cost method for assessing other individuals and gather important information for fitness-relevant decisions, for example in male-male competition or mate choice. During counter-singing interactions in songbirds, males often engage in singing strategies that involve matching the type of an opponent's signal, or adjusting the timing of song production to overlap an opponent. The relative use of matching and overlapping patterns may allow other individuals to gather information about singing males that is important for future territory encounters and reproductive decisions. Yet little is known about the actual relation between these singing behaviors and the individual traits of the singers. Here we used a wireless microphone array to monitor natural counter-singing interactions in a population of great tits during the early breeding season. We explored whether the matching or overlapping behavior is related to individual characteristics that might be relevant for assessing dominance or mate choice. We quantified song matching and overlapping and compared these behaviors to dominance rank, breeding box quality, body condition, plumage ornamentation (size of black breast stripe), male provisioning behavior (feeding hours and rates), and a measure of reproductive success (fledging weight of genetic and foster chicks) for 28 males. We observed no relationship between a focal male's matching or overlapping behavior during interactions and the relative difference between the focal male and its opponent in any of these measures. Moreover, levels of matching and overlapping were not correlated with individual winter dominance rank. Our results thus provide no evidence that eavesdropping on song interactions during the breeding season provides information on relative differences between males for the numerous characteristics we measured. 


\section{Introduction}

Animals make decisions almost constantly, such as where and what to forage (food quality and food patch location), where to live (territory location and migration), and with whom to interact (contests with rivals and mate choice). Observing other individuals, both con- and heterospecific, provides an important source of information for these decisions (Danchin et al., 2004; Goodale et al., 2010; Valone and Templeton, 2002). In species where territory acquisition, social status or mate choice are mediated through competitive interactions, eavesdropping on competitive interactions provides a low-cost opportunity to assess the fighting ability, motivation, or social status of contestants and adjust one's own behavior in response (Cheney and Seyfarth, 1990; Freeman, 1987; Johnson and Akerman, 1998; Oliveira et al., 1998; Silk, 1999).

In many song birds, territorial males frequently engage in singing interactions. During such song contests, males may vary the type of signal they produce and the timing of their vocal signals in response to their rival. Males may produce songs in the silent intervals between their rival's songs (i.e. "song alternating") or they may start singing before the rival's song is finished, thus overlapping the signal of the other in time ("song overlapping"). Males can also respond with either a signal that is different to that of their opponent or they can match the song of the other male in type ("song type matching") (Todt and Naguib, 2000). Both song overlapping and song matching can occur due to chance as a result of several individuals competing for acoustic space and sharing some song types within their repertoires, but could also arise out of intentional processes and have been suggested to provide relevant information to eavesdroppers about the signaler's quality, status, or motivation (Naguib and Mennill, 2010; Todt and Naguib, 2000; Vehrencamp, 2001). Since acoustic signals transmit over large distances, such interactions could provide a low-cost opportunity for other individuals to assess relative differences between interacting males (McGregor, 1993; Valone and Templeton, 2002).

Multiple studies provide evidence that males eavesdrop on singing interactions between others and adapt their own behavior in subsequent encounters (i.e. Hall, Illes, \& Vehrencamp, 2006; Naguib, Amrhein, \& Kunc, 2004; Peake et al., 2002). Male great tits, for example, were able to rank themselves relative to an unknown territory intruder after observing an interaction between that respective male and a third individual they had previously interacted with themselves (Peake et al., 2002). Moreover, female songbirds show a preference for males that overlap or 
match their opponents frequently in interactions and use information gathered by eavesdropping on male-male song interactions to inform reproductive decisions (Amy et al., 2008; GarciaFernandez et al., 2010; Mennill et al., 2002; Otter et al., 1999). Female domestic canaries (Serinus canaria), for example, laid eggs with higher yolk content when exposed to song they had previously heard to be the overlapping song in an interaction (Garcia-Fernandez et al., 2010) and female great tits forayed more often after eavesdropping on an interaction during which their mate was overlapped frequently by a simulated intruder, and mostly into territories of males that had not been overlapped themselves (Otter et al., 1999). In black-capped chickadees (Poecile atricapillus), high-ranking males that were frequency matched and overlapped in an interaction with a simulated intruder were more likely to be cuckolded (Mennill et al., 2002).

Together these studies show that, by attending to relative singing behavior during vocal interactions, both male and female songbirds gain important information that they use to inform subsequent territorial behavior and reproductive decisions. However, the nature of this information is still subject to discussion (Helfer and Osiejuk, 2015; Naguib and Mennill, 2010; Searcy and Beecher, 2011, 2009). Both matching and overlapping have been suggested to be signals of aggressive intent (Naguib and Mennill, 2010; Todt and Naguib, 2000). However, whether males that match and overlap frequently are more successful in territory defense, or of higher social status or whether females may gain benefits from mating with males that match or overlap more frequently (i.e. a higher reproductive success) is still not well understood. Song overlapping, for example, has been shown to reflect parasite exposure in early-life in great tits (Bischoff et al., 2009), but is not associated with winter dominance in black-capped chickadees (Fitzsimmons et al., 2008b; Foote et al., 2008a). Furthermore, most studies exploring the signal value of matching and overlapping have used playback experiments to engage males in simulated territory intrusions with an unknown intruder (reviewed in Naguib \& Mennill, 2010; Searcy \& Beecher, 2009). As a consequence, much less is known about the role of these singing patterns in natural interactions between territory holders, where individuals might interact over larger distances and in situations which might not reflect such an immediate threat (but see for example Fitzsimmons, Foote, Ratcliffe, \& Mennill, 2008a; Foote, Fitzsimmons, Mennill, \& Ratcliffe, 2008).

Here we explored whether overlapping or song type matching in natural song interactions is correlated with the relative difference between contestants in characteristics that might be 
important for males and females to assess individuals when making decisions in future territorial encounters and reproductive choices. Using an wireless microphone array, we quantified matching and overlapping in singing interactions in male great tits during early spring (nest building and early egg laying stage). Additionally, we determined winter dominance rank the winter before the breeding season, quality of the breeding nest box (mean and cumulative weight of fledglings over the past 10 years), body condition of males and parental investment (feeding rate and provisioning hours over one day). To determine whether males (and females paired with them) that type match and overlap more in interactions also have a higher nestling success, we cross-fostered hatchlings between broods and measured fledging weight of both genetic and foster offspring as a measure of nestling success, as this is closely related with first year survival (van Balen, 1973). Additionally, we tested whether song type matching or overlapping in interactions is associated with the size of a male's black breast stripe, a visual ornament which has been suggested to signal social status and individual quality in this species (Järvi and Bakken, 1984; Norris, 1993). Specifically, we tested whether there is a relationship between a focal male's singing strategy during interactions (how often it overlapped and type matched its opponent) and the difference between the focal male and its opponent in these characteristics.

\section{Materials and methods}

\section{Study area and population}

We conducted this study in Westerheide, near Arnhem, the Netherlands, using a long-term study population of great tits. Westerheide is a forest of mixed wood with 228 nest boxes distributed over a $1000 \times 1200 \mathrm{~m}$ area. We caught adult birds during regular roost checks and mist netting session from November 2017 to February 2018. Males were then banded with an individual color code and all birds were equipped with an RFID tag embedded in a leg ring (Eccel Technology LTD, Glenfield, UK). From April to July 2018 we monitored nest boxes about twice a week to record start of egg laying, clutch size, hatching date and fledging success. From late March to mid-April we deployed a microphone array in parts of the study site to record singing interactions between males (see below). Within the areas encompassed by the array we cross-fostered chicks in late April and early May when they were 1-2 days old (day 0 being the day of hatching) as described below. The cross-fostering was part of a brood-size manipulation experiment that was conducted in the remaining part of the study. Brood sizes within the microphone array area were not manipulated when cross-fostering chicks. We caught 
parents using spring traps when chicks were 10 days old and measured weight and tarsus length. We measured weight and tarsus length of chicks when they were 14 days old. Chicks that were not cross-fostered were then also ringed with an individual aluminum ring.

\section{Recordings methods and analysis}

From late March to mid-April (during nest building and the early egg laying stage) we recorded the singing behavior of males using a microphone array consisting of 50 Song Meter SM3 recorders (Wildlife Acoustics, Inc. Maynard, MA, USA) placed in a grid as evenly as possible, with recorders approximately $50 \mathrm{~m}$ apart. We moved the array two times to record singing in three adjoining areas. In total, the three recording sessions allowed us to sample a total area of ca. 34 ha and encompassing the nest boxes of 40 breeding pairs. Recorders were mounted on trees approximately $1.5 \mathrm{~m}$ off the ground. We recorded for five consecutive days in each of the three areas. Recorders were programmed to record for 8 hours each day, starting 1.5 hours before sunrise (from approximately $5 \mathrm{~h}$ to $13 \mathrm{~h}$ local time).

For the analysis of singing interactions between males we divided the total area covered by the array in 15 smaller neighborhoods. For 8 of these neighborhoods, we screened all 5 consecutive days for song interactions. For the remaining neighborhoods we excluded one or more days from the analysis due to high winds, rain or technical difficulties with some recorders. The minimum recording duration included for a neighborhood was 8 hours. We viewed and annotated song interactions using the Syrinx PC sound analysis software (J. Burt, Seattle WA), visualizing spectrograms of recordings from all recorders in and around the respective neighborhood (up to 10 channels). We considered two males to be engaged in a vocal interaction when both males sang at least 2 songs in back-and-forth fashion. We considered an interaction to be concluded when one of the males stopped singing. We only screened for singing interactions after sunrise, thus not including possible interactions during the dawn chorus.

Although we had hoped to triangulate the position of singing birds using time-of-arrival differences in recordings of their songs at different microphones (as in Mennill, Battiston, Wilson, Foote, \& Doucet, 2012; Mennill, Burt, Fristrup, \& Vehrencamp, 2006) this was not possible due to technical problems with the time synchronization of the standalone recorder units in most of our recordings; although recordings were synchronized within less than a second, they were not synchronized on the scale of 10's of seconds as would be required for 
triangulation. Therefore, we relied on an alternative method to determine the position of singing birds. We used the relative amplitude of the same song recorded in different channels to estimate the approximate location of the singer and assigned a song to a specific breeding male whenever the estimated location was close to an occupied nest box. We included only those interactions for which the estimated locations of both singers were close to two distinct nest boxes and no other individual was singing at similar amplitude close to either box. We measured start and end time of each song during an interaction for both males on the same channel to avoid differences between individuals due to small differences in time synchronization between recorders. For each singer in each interaction we also noted the number of possibilities to song type match the opponent. We defined every instance in which an opponent sang a new song type, either because it was the first to sing in the interaction or because it switched song types during the interaction, as an opportunity to match for the other individual. We considered a rival to have matched if he responded to the opponent with the same song type. To determine song types we followed existing song type categorization criteria for great tits (McGregor et al., 1981). We then calculated for each male in every interaction (1) how many of the total number of songs sung by the opponent it overlapped (proportion of songs overlapped). (2) how much of the total singing time by the opponent it overlapped (proportion of singing time overlapped), and (3) how many opportunities to type match the song of the opponent it used (proportion of opportunities to match used). In total, we included 63 interactions in the analysis, including 28 different males and 31 unique dyads.

Song type matching has previously been reported to occur at levels higher than what would be expected if it happened simply due to chance in great tits (Falls et al., 1982; Krebs et al., 1981). We assessed whether overlapping levels observed in these interactions could occur due to chance using the R package SONG (Song Overlap Null model Generator) (Masco et al., 2015). For a given interaction between individuals A and B the SONG package builds randomized performances for A and B independently and then combines them into randomized interactions. Based on these randomized interactions, the program calculates the amount of overlap expected due to chance and generates a P-value, the probability that a randomized interaction will result in an amount of overlap greater than observed. We calculated the expected proportion of overlapped opponent songs for each of the 63 interactions using 1000 randomizations with the "KeepSongOrder" randomization method provided by the package, which preserves the observed song order, song durations and interval durations and only rearranges the intervals to create randomized performances between the respective individuals. Following the methods by 
Masco et al. (2015) we considered individuals as overlapping above or below chance level when the resulting $\mathrm{P}$ values were $\leq 0.025$ or $\geq 0.975$, respectively.

\section{Cross-fostering and nestling success}

In order to separate possible direct and indirect effects of male characteristics on fledgling weight, we cross-fostered chicks between nest boxes within the recording area. Before crossfostering, we weighed all chicks, ranked them according to weight within broods and gave them a down code by selectively removing down feathers to individually identify each chick within a brood. These down codes were visible until chicks were 6 days old, after which we ringed them with individual aluminum rings. We moved chicks on day 1 or 2 (day 0 being the day of hatching) between pairs of broods with the same hatch date and similar brood size, matching chicks based on their weight rank, so that in each brood half of the chicks were cross-fostered and the other half remained in the natal nest box. Within the area encompassed by the recorder array, we cross-fostered in total 17 pairs of broods. For each male for which offspring had been cross-fostered and that was included in the song interaction analysis $(N=22)$, we calculated the mean weight of moved foster offspring and moved genetic offspring when 14 days old as a measure of fledging weight, which is closely correlated with first year survival (van Balen, 1973).

\section{Provisioning behavior}

For nest boxes within the array-recorded area that had been cross-fostered, we also recorded male provisioning behavior continuously during an entire day between day 12 and 14 (day $0=$ hatch date). We quantified provisioning behavior using RFID readers (Dorset ID, Aalten, The Netherlands) with antennas mounted around the nest box opening. We treated reads of the male RFID tag within $17 \mathrm{~s}$ of each other as one visit, because a comparison of RFID data and nest box video recordings has shown that they are likely caused by the respective individual staying in the nest box or near the nest box opening when feeding (L. Zandberg, personal communication). For each male for which we obtained RFID data and that was included in the song interaction analysis $(N=15)$, we quantified the time between the first and the last presumed provisioning visit in hours ("working day duration") and the provisioning rate (number of feeding visits/working day length in hours). 


\section{Breast stripe size}

When catching adults in spring 2018, we took photos of the ventral side of males to quantify the size of the black breast stripe. To take photos we held birds with the crown and legs touching the background (a $1 \mathrm{~cm}^{2}$ grid sheet) and positioned cameras in a standardized distance and angle, mounted on a camera stand (Figuerola and Senar, 2000). All photos were taken with a Coolpix L31 camera (Nikon Corporation, Tokyo, Japan). For each male that was photographed and also included in the song interactions analysis $(N=24)$, we measured breast stripe size in $\mathrm{cm}^{2}$ from where the ventral stripe widens into a throat patch to the posterior end of the stripe with the image analysis software ImageJ v. 1.45s (Abramoff et al., 2004).

\section{Winter dominance score}

In February and early March 2018, we placed cameras at 4 feeding stations to record pairwise dominance interactions at feeding tables. Feeding stations were opened from November 2017 and regularly filled with sunflower seeds until early February 2018, when we closed the stations and only supplied one food source (fat ball) in the center of a wooden feeding table $(50 \times 50$ $\mathrm{cm}$ ) that was placed at the location of each feeding station. We placed cameras next to these tables, recording all birds landing on the table area and attached the food source in the center of the table with a string. Cameras were started between $8 \mathrm{~h}$ and $9 \mathrm{~h}$ in the morning and left to record for 8 hours. We screened the resulting recordings for interactions between males that could be identified using individually color-coded leg rings. In total, we screened 64 hours (7 days recorded in February and 1 day in March) of recordings and tabulated 205 interactions between 41 identified males. We inferred dominance when an individual 1) supplanted or chased an opponent and 2) fed while an opponent waited on the table to access the central food source. To facilitate comparison of males across feeding stations we assigned males a continuous rank score. We calculated for each male the total number of interactions won divided by the total number of interactions he was involved in as well as the total number of opponents he won against divided by the total number of opponents he interacted with. For 7 males we obtained both a dominance rank from interactions at feeding tables and information on singing behavior from song contests during the next spring.

\section{Body condition and breeding nest box quality}

We calculated body condition as the residuals from a linear regression of body weight and tarsus length (measured when parents were caught during spring when chicks were 10 days old) for males involved in song interactions analyzed for which we had tarsus and weight measures 
$(N=23)$. We used the cumulative and mean weight of all chicks (measured when 14 days old) raised in the respective nest box over the last 10 years $(2008-2017)$ as measures for the quality of 27 nest boxes occupied by males included in the song interactions analysis.

\section{Statistical analysis}

We calculated the difference in each of the measured characteristics ( $\Delta$ body condition, $\Delta$ nest box quality, $\Delta$ mean weight of genetic and foster offspring, $\Delta$ feeding rate, $\Delta$ work day duration, $\Delta$ breast stripe size) between opponents for each interacting pair included in the analyzed song interactions. We then tested for an association between the difference between opponents in the respective measure ( $\Delta$ male characteristic) as fixed effect and the proportion of opponent songs overlapped, the proportion of the opponent's singing time overlapped and the proportion of opportunities to match used as dependent variables in a separate model each. We included dyad ID as random factor in each of these models to account for multiple measures for the same pair of males. Since we only had dominance rank scores available for both opponents for one pair of males included in the song interaction analysis, we could not use the difference between opponents for this measure as fixed effect. Instead, we tested for a correlation between the focal male's rank score (of the 7 males for which it was available) as fixed effect and the proportion of opponent songs overlapped, the proportion of the opponent's singing time overlapped and the proportion of opportunities to match used as dependent variables in a separate model each, including male ID as random factor. We used linear mixed models for all analyses with the proportion of opponent songs overlapped and the proportion of the opponent's singing time overlapped as dependent variables. There was only one interaction in which an individual had more than one opportunity to song type match its opponent (and none of them were used), thus the dependent variable 'proportion of opportunity to match used' was binary ( 0 when a male did not match, 1 when the male matched). We used binomial generalized linear mixed models for all analyses including the proportion of opportunities to match used as dependent variable. We fitted all models using the package lme4 (Bates et al., 2015) in R (R Core Team, 2019). Since we ran multiple models with a single predictor variable for each dependent variable (10 models each), we adjusted the $\alpha$ of 0.05 using the Bonferroni correction $\alpha / \mathrm{n}$ to 0.005 . The proportion of opponent songs overlapped was correlated with song rate $(R=0.18, P=0.04)$. We therefore rerun all models including the proportion of opponent songs overlapped as response variable correcting for song rate by adding it as a fixed factor, to exclude the possibility that observed differences between males were related to differences in song rate rather than overlapping. This did not change the results. 


\section{Results}

\section{Frequency of song overlapping and song type matching}

Across 63 counter-singing interactions we recorded from 31 dyads of territorial males, we found that males overlapped on average $25.5 \pm 3 \%$ (mean \pm se) of the songs their opponents sung during an interaction and $11.2 \pm 1 \%($ mean \pm se) of the total singing time of their opponent. In $43 \%$ of the analyzed interactions one or both individuals overlapped at levels lower than expected by chance, in $6 \%$ of interactions one or both individuals overlapped at levels greater than expected by chance; in the remaining interactions, individuals were overlapping at levels not significantly different from chance. In almost all interactions, individuals sang the same song type throughout the entire interaction, resulting in only one opportunity to song-type match, as defined above, for just one of the males in each interaction. In only one out of the 63 analyzed interactions, an individual had more than one opportunity to match its opponent. Males used on average $29.6 \pm 6 \%$ (mean $\pm \mathrm{se}$ ) of their opportunities to match an opponent.

\section{Singing behavior in interactions and other male characteristics}

None of the three measures of male singing behavior (proportion of opponent songs overlapped, proportion of total singing time overlapped by the opponent, and proportion of opportunities to type match the opponent's song used) were associated with the difference between opponents in body condition, nest box quality, breast stripe size, mean weight of genetic and foster offspring and provisioning behavior (Table S1, Figures 1-2 \& Figure S1). Moreover, none of the tested singing measures was associated with the dominance score (proportion of interactions at feeding tables won or proportion of opponents won against) of the focal male (Table S2, Figure 3). We were unable to test whether there was an association between all measures of male singing behavior and all measured male characteristics because of a singular fit of some models. 

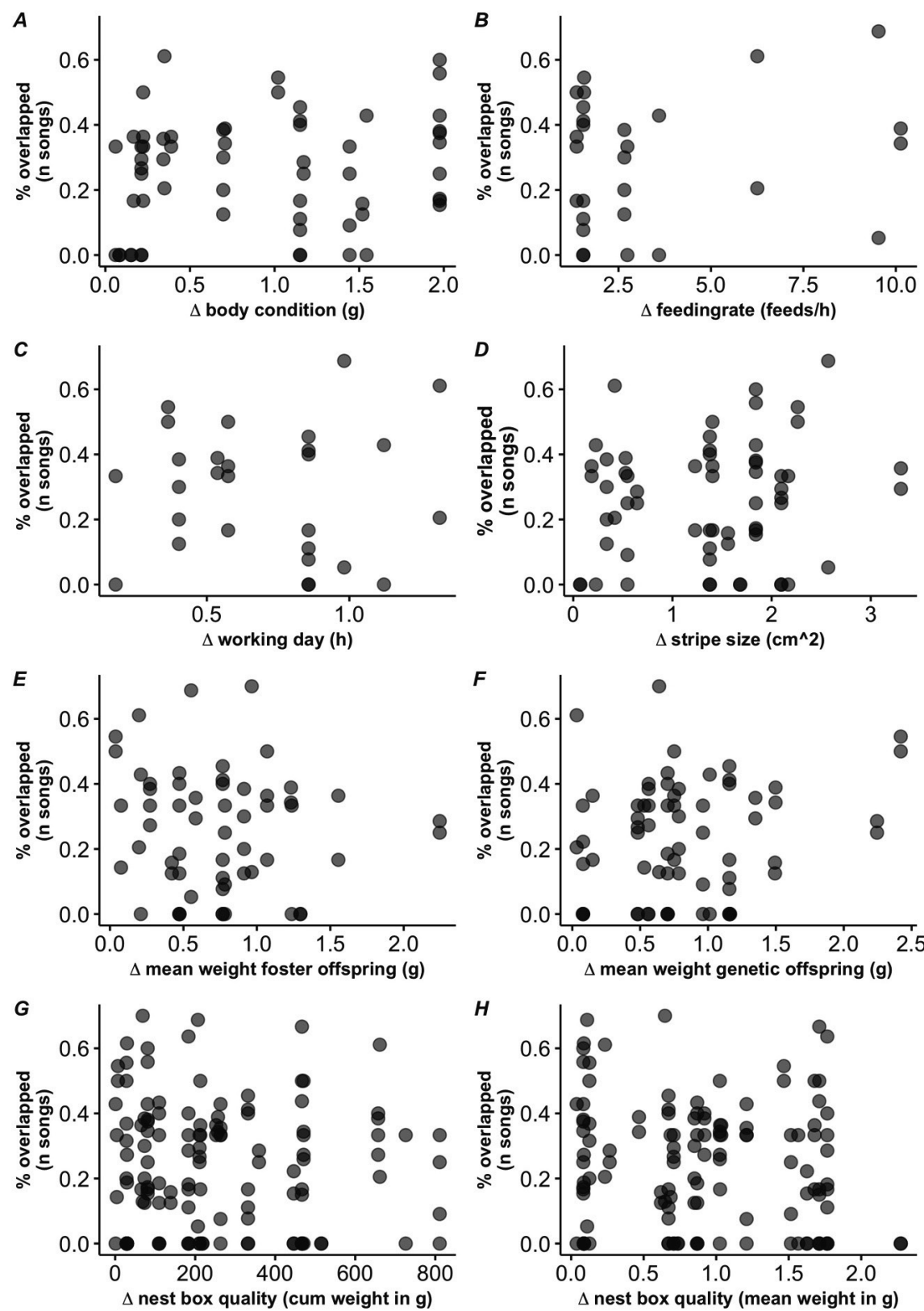

Figure 1 - Proportion of opponent songs overlapped and measures of relative difference between interacting males. There was no correlation between the proportion of opponent songs overlapped by the focal male and the difference between the focal male and the respective opponent ( $\Delta$ ) in body condition, working day duration (time between first and last feed on day of measuring in hours), size of the black breast stripe, mean weight of genetic and foster offspring and nest box quality (estimated as cumulative and mean fledgling weight of all chicks raised in the respective nest box over the last 10 years). We could not test for a correlation between the proportion of overlapped opponent songs and feeding rates (feeds/hour) due a singular fit of the respective model. All points are grey and appear darker when overlapping. 

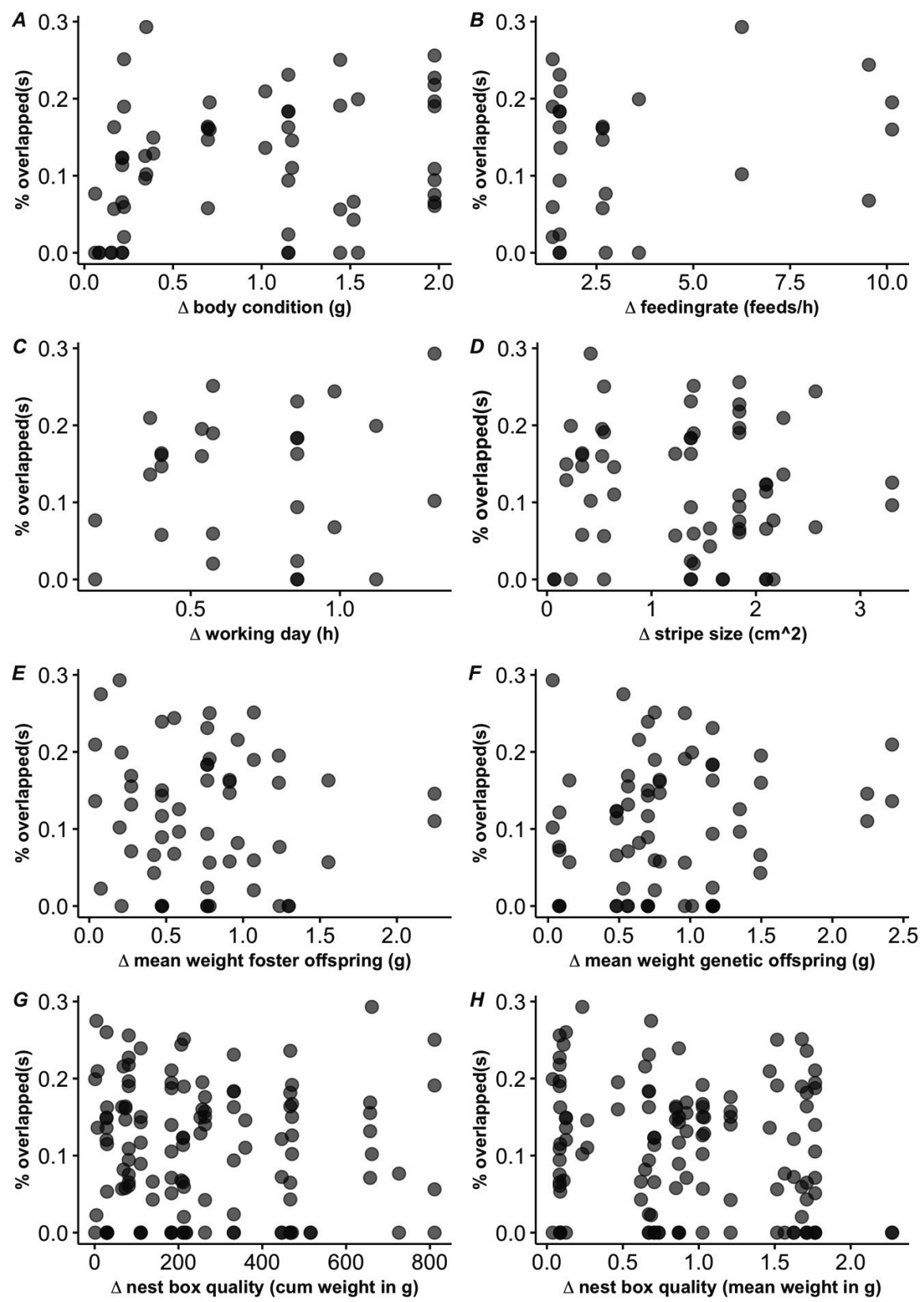

Figure 2 - Proportion of opponent singing time overlapped and measures of relative difference between interacting males. There was no correlation between the proportion of opponent singing time overlapped by the focal male and the difference between the focal male and the respective opponent $(\Delta)$ in the size of the black breast stripe and nest box quality (estimated as cumulative and mean fledgling weight of all chicks raised in the respective nest box over the last 10 years). We could not test for a correlation between the proportion of overlapped opponent singing time and body condition, working day duration (time between first and last feed on day of measuring in hours), feeding rates (feeds/hour) and mean weight of genetic and foster offspring due a singular fit of the respective models. All points are grey and appear darker when overlapping. 

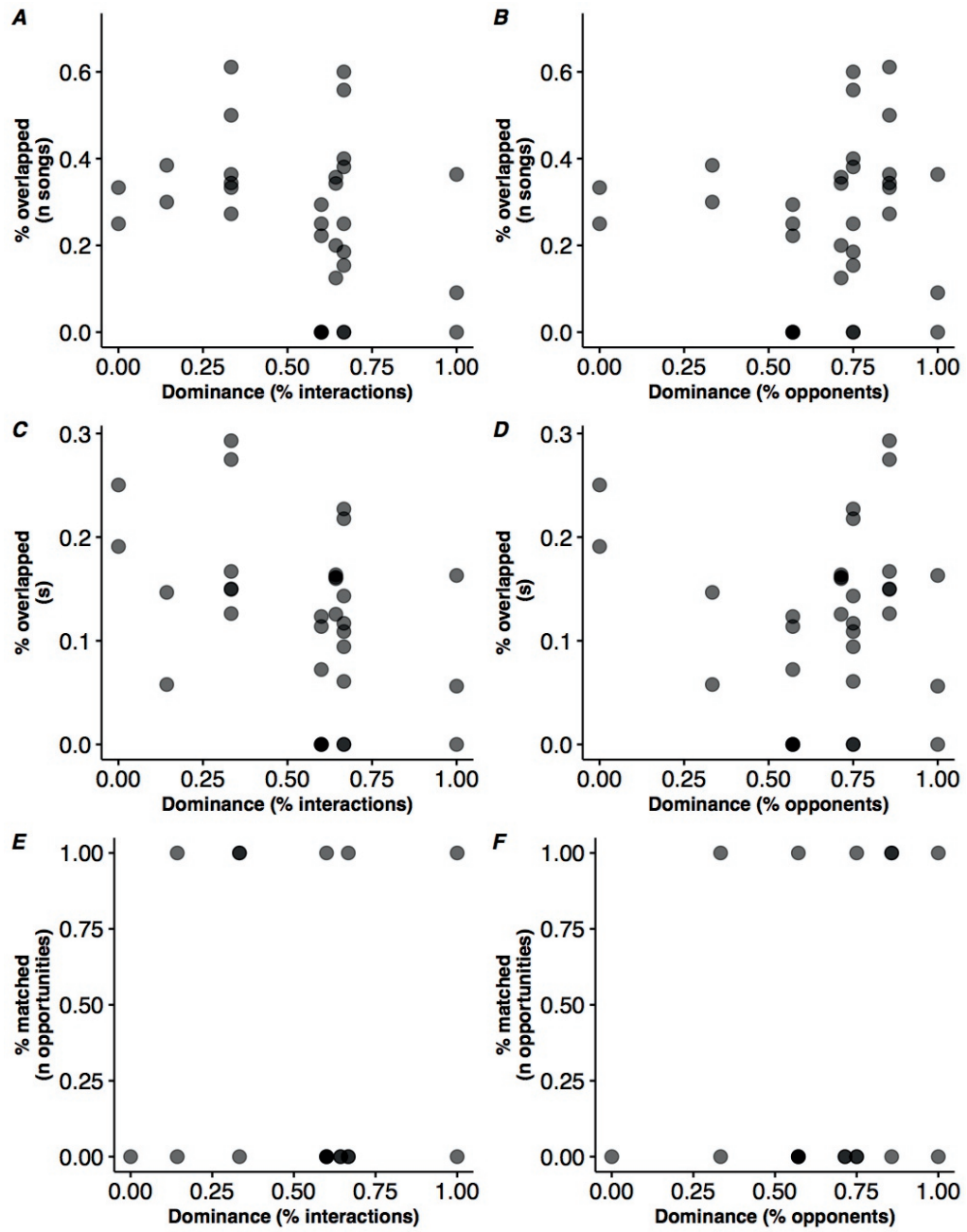

Figure 3 - Overlapping and song type matching in relation to male dominance rank scores. Neither dominance rank scores calculated as the proportion of total interactions $(A \& C)$ nor dominance scores calculated as the proportion of the total number of opponents interacted with $(B \& D)$ correlated with an overlapping measure. We could not test for a relationship between dominance ranks cores and song type matching due to a singular fit of the respective models. All points are grey and appear darker when overlapping. 


\section{Discussion}

Here we explored whether song type matching and song overlapping in naturally occurring, post-dawn chorus song interactions between territorial male great tits reflect relative differences between contestants in several characteristics that may be important for the assessment of rivals and potential mates. We found that neither the level of song type matching nor the level of song overlapping during male-male counter-singing interactions was associated with the difference between the focal male and the opponent in body condition, nest box quality, breast stripe size, provisioning behavior or nestling success. Moreover, levels of song type matching and overlapping were not associated with individual winter dominance rank.

Multiple studies have shown that both male and female songbirds attend to signaling patterns such as song type matching and song overlapping in male-male interactions and derive important information from it, as they changed their behavior in subsequent territorial disputes or reproductive decisions (Garcia-Fernandez et al., 2010; Hall et al., 2006; Mennill et al., 2002; Schmidt et al., 2007). Therefore, we expected that these signaling patterns might reflect relative differences between males in characteristics that would be important for such decision making, i.e. in assessing the quality of mates or the threat posed by rivals. For example, if song type matching or overlapping is a signal of aggressive intent, we might expect males which are subordinate in winter flocks to match and overlap less often overall and especially when interacting with dominant males. Therefore, we also would expect the use of these signaling strategies to reflect individual dominance rank or the relative difference in dominance rank between interactants. Yet, we found no relationship between the use of either singing strategy and individual dominance rank. Song type matching and song overlapping in naturally occurring interactions thus does not seem to signal winter dominance status in the great tit, although it is important to note that our sample size of seven males for this test was small. Moreover, we were not able to control for the distance between a feeding station where an interaction took place and the respective male's territory, due to a singular fit of the respective models when including distance. A previous study in the same population showed that dominance at feeding tables in the winter is highly correlated with the distance between a males' territory and the respective feeding station (Dingemanse and de Goede, 2004), thus including this correction might have changed our results. Previous studies on natural interactions in blackcapped chickadees reported that the level of matching, but not song overlapping, was correlated with winter dominance rank (chickadees engage in frequency-matching rather than song-type matching; Fitzsimmons et al., 2008b; Foote et al., 2008), although there was a non-significant 
tendency in a playback induced interaction with a simulated intruder for males of higher dominance rank to overlap the intruder's song more often (Baker et al., 2012). We also found no relationship between a male's use of song type matching and overlapping in the interactions we studied and the relative difference between him and his opponent in any of the other characteristics we measured. Despite evidence in great tits and other species that these singing behaviors are important in territory defense and assessing rivals in territorial disputes, males in our study that overlapped and matched more frequently did not breed in better quality nest boxes, i.e. boxes that over the last 10 years produced nestlings of higher fledgling weight, which is related to first year survival (van Balen, 1973). Moreover, previous studies showed experimentally that females changed their reproductive decisions (mating strategy in blackcapped chickadees (Mennill et al., 2002); investment in eggs in domestic canaries (GarciaFernandez et al., 2010); male assessment in great tits (Otter et al., 1999)), after eavesdropping on song interactions between males, likely based on information they gathered through attending to the differences in the use of type matching and overlapping between males that were simulated during these experiments. Moreover, great tit males raised in parasite-free nests overlapped a simulated intruder more than males exposed to parasites in early life (Bischoff et al., 2009) and in nightingales males overlapping more in interactions during the time of mate attraction were more likely to be paired later (Kunc et al., 2006). However, our results here do not suggest any potential benefit for females choosing males that type match or overlap more during natural interactions, as neither of these singing patterns was associated with male characteristics that would potentially benefit the female in a direct or indirect way, such as breeding box quality or provisioning behavior. Singing patterns in our study were also not related to differences in males' success in raising heavier nestlings, neither through a male's genetic offspring nor through foster offspring he helped rearing, suggesting that males matching and overlapping more and the females paired with them overall might not achieve a higher fitness.

A possible explanation that we did not find any correlation between song type matching and overlapping and the relative differences between opponents is that we monitored interactions between established neighbors. Such singing patterns might be especially important in interactions with unknown individuals, as many territorial animals react more aggressively to unfamiliar strangers than to familiar neighbors (reviewed in Temeles, 1994) and many songbirds perceive the song of known individuals as less threatening (reviewed in Stoddard, 1996). Studies that found strong reactions to matching and overlapping by males and 
subsequent behavioral changes by eavesdropping males and females were mostly playback studies simulating an unknown territory intruder (reviewed in Helfer \& Osiejuk, 2015; Naguib \& Mennill, 2010; Searcy \& Beecher, 2009). Moreover, most playback studies used to test male and female response to male-male interactions used matching and overlapping levels that were much higher (for example overlapping with $86-100 \%$ in great tits: Dabelsteen, McGregor, Shepherd, Whittaker, \& Pedersen, 1996; Peake et al., 2002) than what we observed in natural interactions in the present study. In the interactions included in our analysis, males overlapped on average $25.5 \pm 3 \%$ (mean \pm se) of songs sung by the opponent and used $29.6 \pm 6 \%$ (mean \pm se) of opportunities to match. This suggests that male great tits song type match and overlap much more sparingly during natural interactions than in playback studies which simulated a territorial intrusion. Matching and overlapping might thus be used by eavesdroppers for assessing unfamiliar rather than familiar individuals. However, in a playback conducted in the same study population (Chapter 3 ) engaging breeding males in an interaction with a simulated, unknown intruder, we did not find any evidence for eavesdropping by either males or females, showing that the effect of overlapping even during territorial intrusions is variable.

Our analysis focused on interactions with males being relatively far apart from each other, as we could not localize exact singing positions through triangulation (Mennill et al., 2006) and identify singers by means other than through their spatial association with a nest box. We were thus limited to measuring interactions where the position of both singers could be estimated by the different amplitude of their songs on several channels and their so estimated positions were clearly associated with distinct nest boxes. We were therefore not able to test whether matching and overlapping would occur more often when birds are close to each other, for example when interacting at territory boundaries, which would be expected if these singing patterns were used more often by males in aggressive contexts (Helfer and Osiejuk, 2015; Searcy and Beecher, 2009). However, a previous study on natural interactions reported no relationship between (frequency-) matching or overlapping levels and the distance between interacting males in black-capped chickadees (Fitzsimmons et al., 2008b).

Matching and overlapping in male-male interactions at other times of the year might still reflect differences between opponents in some of the characteristics we measured. Krebs et al. 1981 reported song type matching levels in great tits to decline from an initial level of $75 \%$ in interactions observed in January to a level of $45 \%$ for interactions observed end of February, when territories were well established (Krebs et al., 1981). The average proportion of matching 
opportunities used in our study, with interactions from late March and early April, was even lower with $35 \%$ and during most interactions, song overlapping occurred at or below levels that could be expected by chance. Monitoring singing interactions between males before territory establishment, when males might be less familiar with each other and motivation during territory disputes is higher, would therefore be necessary to explore within and between individual variation over the season. This would help to clarify whether matching and overlapping levels used by males before territory boundaries are established are different from levels used later in the season and would be related with for example differences in dominance rank or success in acquiring a high-quality territory.

In summary, our results provide no evidence that song type matching and song overlapping during natural male-male interactions in the great tit reflect relative differences between males in a number of individual characteristics that would be relevant for decision making regarding future territorial encounters or in mate assessment. Rather than signaling relative differences between neighboring males, as we studied them here, these singing patterns may be mostly important for males when responding to intruders, as simulated in most playback studies, or between males before territory establishment.

\section{Acknowledgements}

We would like to thank Geldersch Landschap \& Kasteleen for permitting us to conduct fieldwork in Westerheide and Bernice Sepers, Filipe Ribeiro da Cunha, Piet de Goede, Louis van de Kant, Thomas van der Swaluw and Lydia Nieuwe Weme for their help with data collection. This study was permitted by the Dutch legal entity Dier Experimenten Comissie (DEC) no. NIOO-10.05 to $\mathrm{MN}$ and $\mathrm{KvO}$ and no. NIOO 12.02 to $\mathrm{KvO}$. The project was funded by an NWO-ALW open competition grant (824.15.012) to MN. 
Supplement to chapter 4 
Figures
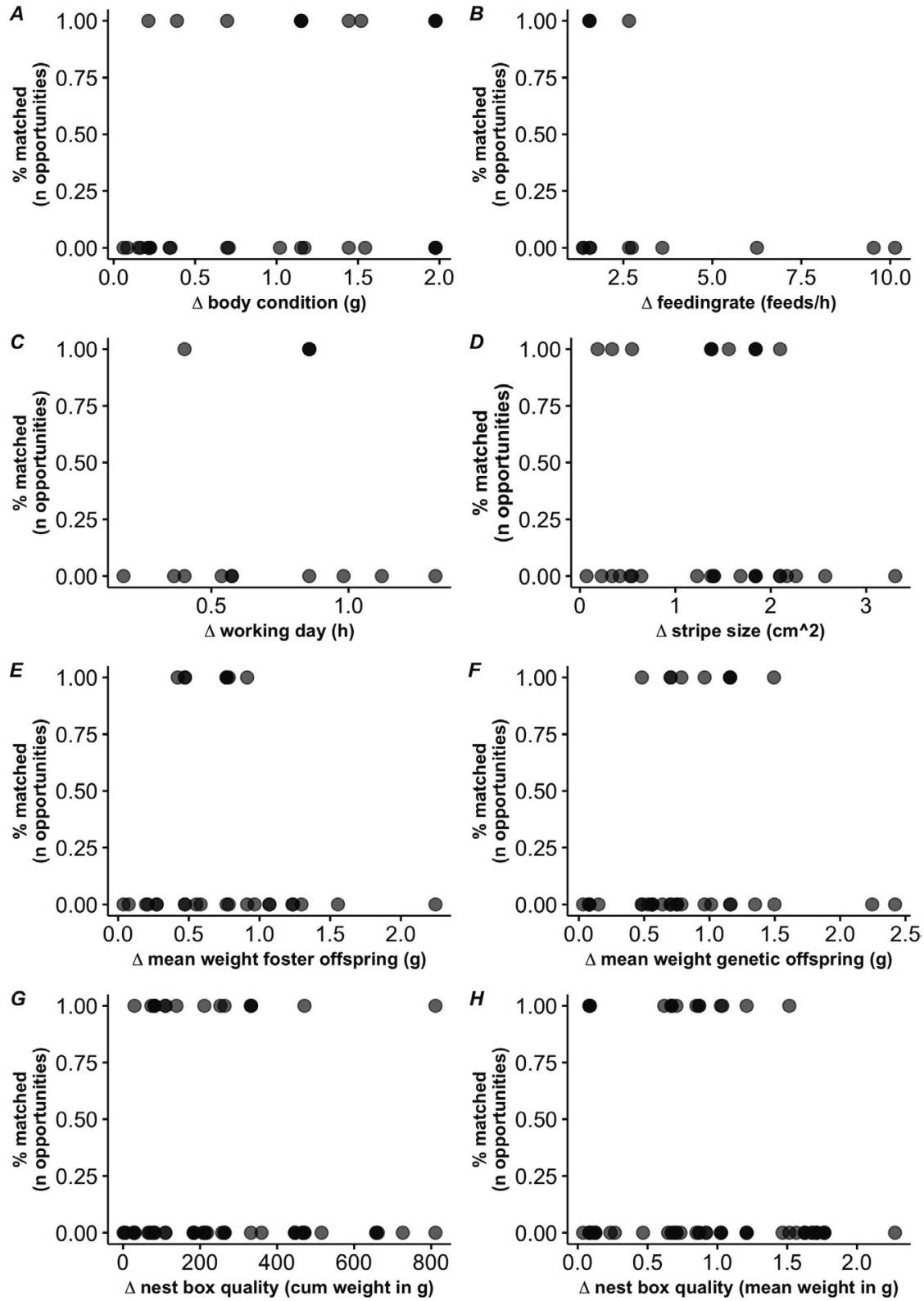

Figure S1 - Proportion of matching opportunities used and measures of relative difference between interacting males. There was no correlation between the proportion of matching opportunities used by the focal male and the difference between the focal male and the respective opponent ( $\Delta$ ) in working day duration (time between first and last feed on day of measuring in hours), feeding rate (feeds/hour), mean weight of genetic and foster offspring and nest box quality (estimated as cumulative fledgling weight of all chicks raised in the respective nest box over the last 10 years). We could not test for a correlation between the proportion of matching opportunities used and body condition, breast stripe size and nest box quality estimated as mean fledgling weight over 10 years due a singular fit of the respective model. All points are grey and appear darker when overlapping. 


\section{Model tables}

Table S1 - Singing behavior of focal male and difference in measured characteristics between opponents. Table lists all (generalized) linear mixed models with the proportion of the number of opponent songs overlapped, the proportion of the opponent's singing time overlapped and the proportion of matching opportunities used by the focal male as dependent variables and the difference in each of the measured male characteristics between opponents as fixed factor. Pair ID was included as random factor. Possible relationships that we were not able to test due to a singular fit of the respective model are denoted as "not tested". The test statistic $\left(\chi^{2}\right)$, degrees of freedom ( $d f)$ and significance ( $p$ value) given were determined using likelihood-ratio tests (LRT) and the number of observations and unique pairs included in the respective dataset are given for each model.

\begin{tabular}{|c|c|c|c|c|}
\hline Model & $\chi^{2}$ & P value & N observations & N pairs \\
\hline \multicolumn{5}{|l|}{ Proportion of opponent songs overlapped } \\
\hline Proportion overlapped $\sim$ body condition & 0.78 & 0.38 & 60 & 19 \\
\hline Proportion overlapped $\sim$ working day & 0.00 & 0.96 & 28 & 9 \\
\hline Proportion overlapped $\sim$ feeding rate & \multicolumn{4}{|c|}{ not tested } \\
\hline Proportion overlapped $\sim$ breast stripe size & 0.94 & 0.33 & 62 & 20 \\
\hline Proportion overlapped $\sim$ weight genetic offspring & 0.28 & 0.60 & 56 & 19 \\
\hline Proportion overlapped $\sim$ weight foster offspring & 0.65 & 0.42 & 56 & 19 \\
\hline Proportion overlapped $\sim$ nest box quality (cumulative) & 0.50 & 0.48 & 124 & 30 \\
\hline Proportion overlapped $\sim$ nest box quality (mean) & 1.85 & 0.17 & 124 & 30 \\
\hline \multicolumn{5}{|l|}{ Proportion of opponent singing time overlapped } \\
\hline Proportion overlapped $\sim$ body condition & \multicolumn{4}{|c|}{ not tested } \\
\hline Proportion overlapped $\sim$ working day & \multicolumn{4}{|c|}{ not tested } \\
\hline Proportion overlapped $\sim$ feeding rate & \multicolumn{4}{|c|}{ not tested } \\
\hline Proportion overlapped $\sim$ breast stripe size & 0.11 & 0.74 & 62 & 20 \\
\hline Proportion overlapped $\sim$ weight genetic offspring & \multicolumn{4}{|c|}{ not tested } \\
\hline Proportion overlapped $\sim$ weight foster offspring & \multicolumn{4}{|c|}{ not tested } \\
\hline Proportion overlapped $\sim$ nest box quality (cumulative) & 0.50 & 0.48 & 124 & 30 \\
\hline Proportion overlapped $\sim$ nest box quality (mean) & 2.17 & 0.14 & 124 & 30 \\
\hline \multicolumn{5}{|l|}{ Proportion of opportunities to match used } \\
\hline Proportion matched $\sim$ body condition & \multicolumn{4}{|c|}{ not tested } \\
\hline Proportion matched $\sim$ working day & 0.00 & 0.98 & 14 & 9 \\
\hline Proportion matched $\sim$ feeds per hour & 2.05 & 0.15 & 14 & 9 \\
\hline Proportion matched $\sim$ breast stripe size & \multicolumn{4}{|c|}{ not tested } \\
\hline Proportion matched $\sim$ weight genetic offspring & 0.21 & 0.65 & 28 & 19 \\
\hline Proportion matched $\sim$ weight foster offspring & 0.35 & 0.42 & 28 & 19 \\
\hline Proportion matched $\sim$ nest box quality (cumulative) & 0.21 & 0.65 & 65 & 30 \\
\hline Proportion matched $\sim$ nest box quality (mean) & \multicolumn{4}{|c|}{ not tested } \\
\hline
\end{tabular}


Table S2 - Focal male singing behavior and winter dominance rank. Table lists all linear mixed models with the proportion of the number of opponent songs overlapped, the proportion of the opponent's singing time overlapped and the proportion of matching opportunities used by the focal male as dependent variables and its dominance score (proportion of interactions won and proportion of opponents won against) as fixed factor. Individual ID was included as random factor. We were not able to test for an association between dominance rank scores and the proportion of matching opportunities used due to a singular fit of the respective models. The test statistic $\left(\chi^{2}\right)$, degrees of freedom ( $\left.d f\right)$ and significance ( $p$ value) given were determined using likelihood-ratio tests (LRT). Models are based on a dataset of 32 observations including seven males.

\begin{tabular}{|c|c|c|c|c|}
\hline Model & Estimate & se & $\chi^{2}$ & P value \\
\hline Proportion song overlapped $\sim$ dominance (n interactions) & -0.22 & 0.13 & 2.81 & 0.09 \\
\hline Proportion songs overlapped $\sim$ dominance (n opponents) & -0.02 & 0.16 & 0.01 & 0.93 \\
\hline Proportion singing time overlapped $\sim$ dominance ( $\mathrm{n}$ interactions) & -0.13 & 0.07 & 3.63 & 0.06 \\
\hline Proportion singing time overlapped $\sim$ dominance (n opponents) & -0.06 & 0.08 & 0.65 & 0.42 \\
\hline Proportion matched $\sim$ dominance (n interactions) & not tested & & & \\
\hline Proportion matched $\sim$ dominance (n opponents) & not tested & & & \\
\hline
\end{tabular}






\section{CHAPTER 5}

Foraying activity to other territories is associated with pair personality in the great tit (Parus major)

Nina Bircher, Lysanne Snijders, Marc Naguib, Kees van Oers In preparation 


\section{Abstract}

In many territorial species individuals make movements off-territory. Such extra-territorial forays often are visits into others' territories and entail a variety of potential benefits, such as gain of additional social and environmental information, food or opportunities for extra-pair copulations. They also can entail costs, such as leaving the own territory vacant, aggressive encounters, or increased predation risk. Previous studies have shown that spatial, social, and risk-taking behavior is associated with individual personality, yet, whether or not personality traits are also linked to foraying behavior remains unknown. Moreover, during the breeding season, an individual's foraying behavior may not only be influenced by its own personality but also by that of its mate. Here, we determined the relationship between exploratory behavior, an established proxy for a personality trait, and extra-territorial foraying behavior in a population of great tits (Parus major). For two consecutive years, we used an automated radio tracking system to continuously monitor movements throughout the breeding season, when birds are paired and actively defend territories. In contrast to our expectations, exploratory behavior was not associated with an individual's foraying activity. However, faster exploring males made more forays when paired with a slow exploring female, whereas female foraying was not associated with her mate's exploratory behavior. Our findings suggest that males tradeoff the relative costs and benefits of intruding other territories differently, depending on their own personality in combination with that of their partner. As mate personality traits apparently associate with movement behavior, which in turn can greatly affect territory maintenance, social encounters, access to resources, and predation risk, our findings also shed new light on the potential consequences of personality-dependent mate choice. 


\section{Introduction}

In many species, individuals defend resources such as food, mates or breeding places, within a territory, yet individuals also make movements beyond their territory boundary ("extraterritorial forays") and intrude into the territory of others (Celis-Murillo et al., 2017; Mayer et al., 2017; Messier, 1985; Naguib et al., 2001; Young and Monfort, 2009). Sampling of and intrusions into territories by individuals that are not holding a territory yet themselves ("floaters") provides some obvious benefits such as the possibility to take-over a territory or information gathering (Amrhein et al., 2004a; Bruinzeel and van de Pol, 2004). In oystercatchers (Haematopus ostralegus) for example, floater individuals that intruded in specific territories more often, were later more successful in occupying those territories when they became vacant, suggesting that familiarity with an area gained through intrusions is important for territory acquisition (Bruinzeel and van de Pol, 2004). Yet, the benefits of such off-territory movements for current territory holders are still not well understood. Forays of current territory holders may have multiple, non-exclusive purposes. These individuals may also foray off-territory to gather information to aid in future breeding habitat selection. Collard flycatchers (Ficedula albicollis), for example, appear to use parental activity at prospected sites as a source of public information to assess reproductive success (Doligez et al., 2004a, 2002). In addition to gaining information for habitat selection purposes, forays into others' territories may also be used to gather information about the resident territory holder. In song birds for example, where male song often signals individual quality, motivation or status (Gil and Gahr, 2002), forays have been shown to be associated with the residents' singing. In nightingales (Luscinia megarhynchos), the timing of prospecting territories by non-resident females peaks at night when only unmated males are singing while for males it peaks at dawn when all resident males are singing (Amrhein et al., 2004a; Roth et al., 2009). In great tits (Parus major) resident males visit territories of more elaborately singing resident males more often, whereas females were more likely to stay away (Chapter 2). This latter study also included the 'silent' movements at times with no or little singing activity, and so revealed dawn song in this species to be associated with movement in opposite ways than expected based on the observed immediate male deterring and female attraction responses during simulated territory intrusions (Snijders et al., 2017a), potentially because forays serve a different, i.e. information-gathering, function. Apart from such presumably general information gathering, territorial individuals may also foray specifically to forage for additional food: Yearling wolves (Canis lupus) for example, undertook more extra-territorial movements during times of low prey abundance (Messier, 1985). However, a study in Northern Cardinals (Cardinalis cardinalis) found no 
effect of food supplementation on extra territorial foraying behavior of females. Instead, there was a tendency for supplemented females to foray more often, which would contradict the idea that foraying serves the search for additional food sources (Humbird and Neudorf, 2008). Another function for extra-territorial forays into neighboring territories that has often been suggested is the search for potential extra-pair mates. However, while some studies (Double and Cockburn, 2000; Kleven et al., 2006; Schlicht et al., 2015) have provided support for the idea that males and females foray into others' territories to actively search for extra-pair copulations, others have found no links between foraying activity and extra-pair mating patterns (Celis-Murillo et al., 2017; Stutchbury, Pitcher, Norris, Tuttle, \& Gonser, 2005; Chapter 2).

Next to potential benefits, foraying off-territory may also entail costs. Moving through unfamiliar areas can lead to a higher predation risk (Larsen and Boutin, 1994; Metzgar, 1967), loosing stored food to pilfering (Gerhardt, 2005) higher physiological stress levels (Young and Monfort, 2009), lower nest attendance and, as a consequence, higher risk of nest predation (Schmidt and Whelan, 2005). Individuals may thus differ in foraying activity, depending on how they trade-off the relative costs and benefits of leaving their own territory. Foraying activity, for example, has previously been shown to vary with sex and body size (Schlicht et al., 2015), age (Celis-Murillo et al., 2017; Kleven et al., 2006), and social status (Mayer et al., 2017), and thus may entail different costs depending on individual traits or status.

Likely, but less studied, traits affecting an individual's propensity to foray off-territory include consistent behavioral differences (personality) associated with the likelihood to take risks (van Oers et al. 2005). Such consistent variation in behavior between individuals across context or time has been observed in many species, with some individuals consistently showing more aggressive, more bold or more exploratory behavior than others (Sih et al., 2004; van Oers and Naguib, 2013). Several studies have shown that variation in personality is important for individual movement patterns and is associated with migratory tendency, dispersal or foraging behavior (Boon et al., 2008; Chapman et al., 2011; Cote and Clobert, 2007; Nilsson et al., 2010; Wilson and McLaughlin, 2007). Fast and slow exploring birds, for instance, have been shown to differ how far they travel to search for prey (van Overveld and Matthysen, 2010) and in many species faster personalities disperse more readily and further (Chapman et al., 2011; Cote and Clobert, 2007). 
Personality traits have also been shown to be associated with how individuals use social and environmental information: less bold or slower exploring individuals have been shown to more likely base foraging decisions on available social information (Kurvers et al., 2010), pay attention to companions (Kurvers et al., 2009; van Oers et al., 2005), and to continuously collect environmental information on food availability (van Overveld and Matthysen, 2013), while bolder individuals seem to be following a more trial and error strategy (Smit and van Oers, 2019). Additionally, previous studies showed that social connectivity can be associated with personality (Aplin et al., 2013; Godfrey et al., 2012; Picq et al., 2019; Snijders et al., 2014). In great tits, among both territorial birds (Snijders et al., 2014), and birds in foraging winter flocks (Aplin et al., 2013) social connectivity varied with personality, with more exploratory birds showing a higher connectivity. Especially in territorial populations, higher social connectivity may, at least partly, be driven by personality effects on movement patterns. For example, the varying social network connectivity shown in the study by Snijders et al. (2014) may be modulated by personality dependent forays off territory, since at least one of two individuals in an encounter needs to leave its territory.

Movement patterns may not only be influenced by an individual's own personality but also that of its social relations (Kurvers et al., 2009; van Oers et al., 2005). In species with pair-bonds, foraying behavior may depend on the personality of its breeding partner. In great tits, for instance, pairs of extreme and similar personality traits were shown to be more likely to have extra-pair offspring in their nest (van Oers et al., 2008). Moreover, pairs with similar personality traits were shown to have a higher reproductive success, which could be explained by such partners having similar provisioning rules and strategies which might lead to a higher pair coordination (Both et al., 2005; Mutzel et al., 2013; Schuett et al., 2011). Such compatibility between members of a pair might also affect extra-territorial foraying. Given that partners generally affect each other's behavior as they often need to synchronize activities, it can well be expected that partner personality also has effects on an individual's spatial behavior.

Although there is accumulating evidence that personality traits are an important factor for explaining movement patterns in general, the role of such consistent individual differences in movement has been primarily studied in the context of dispersal and foraging tactics (Spiegel et al., 2017) and how personality might affect individual extra-territorial foraying is still poorly understood. Here, we determined whether foraying into neighboring territories is related to personality in great tits. Personality may affect how individuals prioritize motivation and risks 
associated with extra territorial forays with faster exploring, bolder individuals being more likely to cross territory boundaries and thus have more opportunities to gather information on nearby habitat and encounter conspecifics, but also face higher risks. Consequently, we expected that foraying activity would be associated with the exploration behavior of an individual with faster exploring individuals also foraying off-territory more often. Since individual foraying activity could also be influenced by partner personality, we additionally tested whether partner personality is associated with foraying activity. We used an automated radio-tracking system to quantify intrusions of both males and females into other territories ("forays") throughout the breeding season and tested birds for exploratory behavior, an operational measure for personality in this species (van Oers and Naguib, 2013). Specifically, we determined whether individual foraying activity into neighboring territories is associated with an individual's personality, its mate's personality or their combination. 


\section{Materials and methods}

\section{Study area and population}

We collected all data for this study in 2016 and 2017 in Westerheide, a mixed wood area near Arnhem, the Netherlands, with 228 nest boxes distributed over approximately 1000 x $1200 \mathrm{~m}$. From early April to the end of June, we routinely checked nest boxes in the area about twice a week to monitor the initiation of nest building, egg laying, hatching date and fledging success. We caught adult great tits using spring traps when chicks were 10 days old (day 0 being the day of hatching) and measured their tarsus length and weight. We ringed great tit nestlings with an individual aluminum ring when they were 14 days old and measured their tarsus length and weight.

\section{Exploratory behavior}

Using a standard procedure, all newly caught great tits in Westerheide are tested for exploratory behavior with a novel environment test. Exploratory behavior has a large heritable and repeatable component and is a measure for individual tendency to explore a novel environment in wild great tits (Dingemanse et al., 2002; van Oers et al., 2004). Outside the breeding season, birds are caught in their nest boxes during routine roosting checks in the evening or with mist nets during the day and brought to the aviary facilities at the Netherlands Institute of Ecology (NIOO-KNAW). Here, we house birds in individual cages ( $0.9 \times 0.4 \mathrm{~m}$ and $0.5 \mathrm{~m}$ high), and provide water, sunflower seeds and mealworms at libitum. The next day, each individual is tested for exploratory behavior in a novel room ( $4.0 \times 2.4 \mathrm{~m}$ and $2.3 \mathrm{~m}$ high) containing five artificial trees. After the bird enters the room, facilitated by darkening the holding cage we record all movements within (hops) and between (flights) trees for two minutes, which are then used to calculate an individual exploration score ranging from low (slow explorer) to high (fast explorer) on a continuous scale. Birds are weighed, measured and ringed after testing and released close to the place where they were caught the day before.

\section{Radio tracking}

We used the automated radio-tracking system "Encounternet" (Encounternet LLC, Portland, USA) to track movements of male and female great tits during the breeding seasons in 2016 and 2017. Encounternet consists of roaming nodes (tags) and base nodes (receivers) and allows to track multiple individuals simultaneously (Mennill et al., 2012b; Snijders et al., 2017b). Tags are programmed to send out a signal with an individual ID every 5 seconds and receivers within 
range record tag ID, time stamp and a signal strength indication value for every transmitted signal. In 2016, we tagged 79 birds (41 females and 38 males) during a roost check on the $22^{\text {nd }}$ of March. We caught birds at night in their nest boxes and equipped them at the field site with an Encounternet tag using a leg loop harness and brought them back to their nest box. Following the same procedure, we tagged 84 birds ( 54 females and 30 males) on the $24^{\text {th }}$ of March in 2017. We placed receivers in trees close to nest boxes at a height of approximately $2 \mathrm{~m}$ to monitor tagged birds in the vicinity of nest boxes. In 2016, we distributed receivers at every nest box within a smaller part of the study site to track movements from before nest building started until the first days of chick rearing. Overall, 58 of the nest boxes were later used by great tit pairs, of which for 38 nest box areas, we successfully collected tracking data. In 2017, we distributed receivers throughout the whole study site, placing one close to a nest box as soon as nest building was observed. In total, we successfully collected tracking data for 72 nest box areas used by great tit pairs in 2017 covering the time from the start of nest building until the first days of incubation.

We used the signal strength values logged by receivers for each tag signal to estimate the distance between tags and receivers, using a signal strength vs. distance regression based on 10 transects measured throughout the study site in 2016 (for details see Bircher et al. 2020). We subsequently excluded all signals that were determined to be further than $15 \mathrm{~m}$ away from a receiver at a nest box used by great tits for breeding. We took $15 \mathrm{~m}$ as a threshold to assign a signal to a nest box area, as some nest boxes are only $30 \mathrm{~m}$ apart. We used a sliding window approach to determine whether a bird was present in the area around a nest box, treating any individual as present as long as its tag was logged at least 3 times during any 30 second window (i.e. the respective receiver logged $50 \%$ of all signals a tag sent out within any 30 second window). Anytime a bird was present in the area around a nest box that was not its own breeding box, we considered to be a foray into the core of that territory.

We quantified foraying activity of individuals by summarizing the number of forays made per day as well as the number of different nest box areas visited on forays per day. For any tracking day during which no foray was recorded, we added a zero to the dataset. We collected tracking data continuously from the end of March to the end of May in both years, excluding the day after tagging. We thus covered most of the nest building, egg laying, incubation and chick rearing stage. Tracking data from 2016 consisted of a total number of 25,859 forays $(10,322$ by 34 females and 15,537 by 32 males) to 38 monitored nest box areas (Bircher et al., 2020 - 
Chapter 2). In 2017, tracking data included 76,792 forays (37,990 by 50 females and 38,802 by 28 males) to 72 monitored nest box areas. 19 individuals were tracked in both 2016 and 2017 and thus in total, the data set from both years included 125 unique individuals, 84 of which were tested for exploratory behavior. We had data available for 27 unique breeding pairs with both the female and male tagged (18 pairs in 2016 and 9 pairs in 2017). Only one individual was included in this pair dataset in both years, but with a different mate.

\section{Statistical analysis}

We analyzed all data in R v.3.6.2 (R Core Team, 2019) with the package lme4 (Bates et al., 2015) and glmmTMB (Brooks et al., 2017). We checked model assumptions with diagnostic plots produced with the package DHARMa (Hartig, 2019). We first fitted a poisson GLMM with log link function for all models. The respective diagnostic tests provided by the DHARMa (Hartig, 2019) package suggested zero-inflation and over dispersion for the poisson GLMMs with the number of forays per day as dependent variable. We therefore used zero-inflated negative binomial GLMMs with a log link function and the number of individual forays per day as dependent variable instead. For models with the number of nest box areas visited per day as dependent variable, we used poisson GLMMs with a log link function. To test whether individual foraying behavior is associated with the focal exploratory score or the interaction between exploratory score and sex, we fitted models with number of forays made per day or number of nest box areas visited per day as dependent variable and exploratory score (continuous variable), sex and their interaction (exploratory score*sex) as fixed factors. To test whether individual foraying behavior is associated with mate exploratory score or the interaction between mate exploratory score and the focal individual's own exploratory score we fitted models for males and females separately, with the number of forays or nest box areas visited per day as dependent variable and exploratory score of the focal individual, exploratory score of the focal individual's mate and their interaction (exploratory score focal*exploratory score mate) as fixed factors. In all of the above-mentioned models, we included ID of the focal individual as random factor to account for multiple observations per individual. In addition, we included year, the average distance between the nest box of the focal individual and all other nest box areas with receivers and tagged individuals in the same year as well as lay date relative to the population median as fixed factors to account for possible differences in foraying behavior between years, an individuals' central or edge breeding location and early or late breeding start, respectively. We determined significance with likelihood ratio tests and used stepwise backwards elimination, starting with the highest order interaction or least significant 
variable, to obtain final models. Year, lay date relative to the population median and average distance to other boxes were always kept in the final model independent of significance.

\section{Results}

Foraying behavior, sex and exploratory score of focal individuals

Foraying activity (both the number of forays and the number of nest box areas visited per day) differed between the sexes, with males making fewer forays per day and visiting fewer nest box areas per day than did females. Foraying activity was not associated significantly with the exploratory score of focal individuals or with the interaction between the exploratory score and sex of focal individuals (number of forays: exploratory score*sex: $\chi^{2}=0.77, P=0.38$, exploratory score: $\chi^{2}=0.10, P=0.77$, sex: $\chi^{2}=110.45, P=<0.001$; number of nest box areas visited: exploratory score*sex: $\chi^{2}=0.007, P=0.93$, exploratory score: $\chi^{2}=1.36, P=0.24$, sex: $\chi^{2}=14.57, P=<0.001 ; N=4267$ observations, 71 individuals; for full model tables see supplementary Table S1-S2).

Foraying behavior, exploratory score of focal individual and its mate

Foraying behavior of males was associated with the interaction between their own and their mate's exploratory score: Fast exploratory males made more forays (Figure 1A) and tended to visited more nest box areas (Figure 1B) per day when paired with slow exploring females (number of forays: exploratory score male*exploratory score mate: $\chi^{2}=5.12, P=0.02$; number of nest box areas visited: exploratory score male*exploratory score mate: $\chi^{2}=3.81, P=0.051$; $N=1113$ observations, 19 males; for full model tables see Table 1-2). Foraying behavior of females was not explained by variation in their own exploratory score, their mate's exploratory score or the interaction between the two (number of forays: exploratory score female*exploratory score mate: $\chi^{2}=0.004, P=0.95$, exploratory score mate: $\chi^{2}=0.01, P=$ 0.93, exploratory score female: $\chi^{2}=0.57, P=0.45$; number of nest box areas visited: exploratory score female*exploratory score mate: $\chi^{2}=0.07, P=0.8$, exploratory score mate: $\chi^{2}=0.13, P=0.71$, exploratory score female: $\chi^{2}=1.39, P=0.24 ; N=1471$ observations, 26 females). 

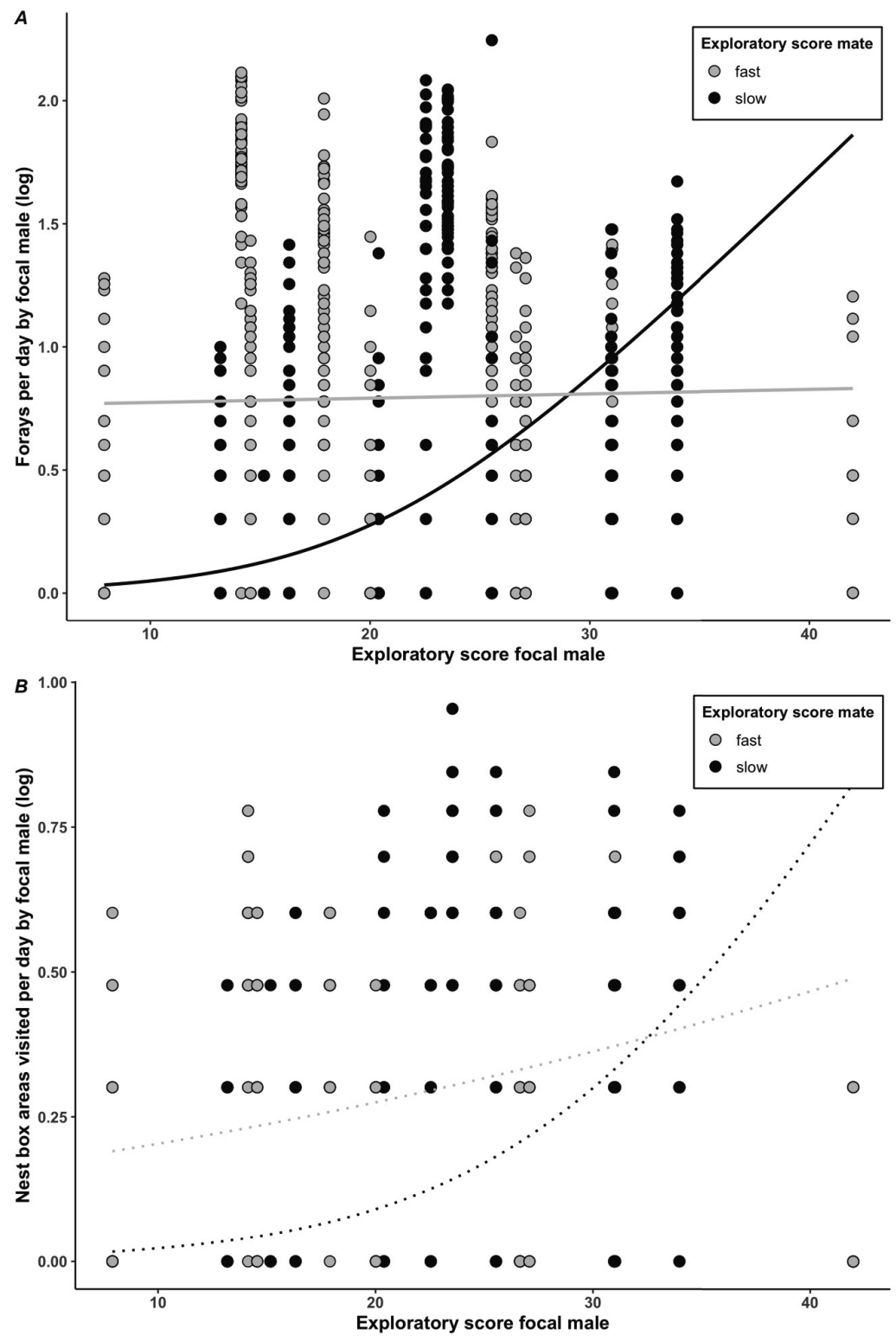

Figure 1 - Foraying activity of focal males, their own and their mate's exploratory score. The number of forays made $(A)$ and number of nest box areas visited on forays $(B)$ per day by focal males $(N=19)$ was correlated with their own and the exploratory score of their mate (interaction term): When paired with slow exploring females, fast exploring males undertook more forays per day (Table 1) and tended to visit more different areas (Table 2). Exploration scores of mates were included as continuous variable in the respective model, but are here represented as slow or fast, depending on whether scores were smaller or larger than the median exploration score of 22.2. Lines depict model predictions for males with a slow (exploration score equals 14.1, the value of the first quartile) or a fast (exploration score equals 28.0, the value of the third quartile) mate. We added 1 to counts of forays per day in order to present them on a log scale. Data based on 1113 observation days and 19 focal males, all observations with exactly the same value are plotted as one point. 
Table 1 - Number of forays per day and exploratory score of the focal male and its mate. Table lists all factors included in a zero-inflated negative binomial GLMM with log link function. The dependent variable was the number of forays per day per individual $(N=1113$ observations, 19 individuals). Individual ID was included as random factor (var $\pm s d: 1.65 \pm 1.28)$. Using backward elimination, the estimate and standard error of the last model in which a factor was included are given. The test statistic $\left(\chi^{2}\right)$, degrees of freedom (df) and significance ( $p$ value) given were determined using likelihood-ratio tests (LRT). The factors year, distance (average distance of the focal individual's nest box location to other nest boxes with receivers or tagged individuals) and relative lay date of the focal individual's brood were included in the last model, independent of significance.

\begin{tabular}{|c|c|c|c|c|c|}
\hline & Estimate & se & $x^{2}$ & df & P value \\
\hline Intercept & -7.57 & 3.59 & - & - & - \\
\hline Exploratory score focal $*$ exploratory score mate & -0.01 & 0.01 & 5.12 & 1 & 0.02 \\
\hline Exploratory score focal & 0.40 & 0.15 & - & - & - \\
\hline Exploratory score mate & 0.41 & 0.16 & - & - & - \\
\hline Year (2017) & 0.57 & 0.97 & - & - & - \\
\hline Distance & -3.73 & 3.95 & - & - & - \\
\hline Relative lay date & -0.04 & 0.04 & - & - & - \\
\hline
\end{tabular}

Table 2 - Number of nest box areas visited per day and exploratory score of the focal male and its mate. Table lists all factors included in a poisson GLMM with log link function. The dependent variable was the number of forays per day per individual ( $N=1113$ observations, 19 individuals). Individual ID was included as random factor (var $\pm s d: 0.65 \pm 0.80$ ). Using backward elimination, the estimate and standard error of the last model in which a factor was included are given. The test statistic $\left(\chi^{2}\right)$, degrees of freedom ( $\left.d f\right)$ and significance ( $p$ value) given were determined using likelihood-ratio tests (LRT). The factors year, distance (average distance of the focal individual's nest box location to other nest boxes with receivers) and relative lay date of the focal individual's brood were included in the last model, independent of significance.

\begin{tabular}{|c|c|c|c|c|c|}
\hline & Estimate & se & $x^{2}$ & df & P value \\
\hline Intercept & -0.32 & 0.26 & & & \\
\hline Exploratory score focal $*$ exploratory score mate $($ scaled) & -0.58 & 0.29 & 3.81 & 1 & 0.051 \\
\hline Exploratory score focal (scaled) & 0.66 & 0.22 & - & - & - \\
\hline Exploratory score mate (scaled) & 0.77 & 0.19 & - & - & - \\
\hline Year (2017) & -0.20 & 0.61 & - & - & - \\
\hline Distance (scaled) & -0.25 & 0.31 & - & - & - \\
\hline Relative lay date (scaled) & -0.39 & 0.23 & - & - & - \\
\hline
\end{tabular}




\section{Discussion}

We here show that neither male nor female foraying behavior was associated with individual exploratory behavior. Yet, male foraying behavior was related to the combination of their own and their partner's exploratory score, while female foraying behavior was not associated with that of their partner. Fast exploring males paired to slow exploring females made more forays into other territories per day and tended to visit more areas per day. In contrast, when paired to fast exploring females, fast and slow exploring males did not differ much in their foraying activity.

In contrast to our expectations, foraying activity (both the number of forays per day and the number of different areas visited per day) into other territories was not associated with the exploratory score of focal individuals, neither males nor females. Our results thus suggest that faster and slower explorers do not prioritize potential benefits and costs associated with foraying differently. However, it is possible that the forays we monitored here were not perceived as a high risk in the first place: although both males and females in our study forayed frequently, they visited few other territories per day. Unless birds were able to very quickly pass surrounding territories, this suggests that both males and females forayed mostly into close neighboring territories with which they may be relatively familiar. Familiarity between neighboring great tits is associated with joining in neighbor nest defense and is positively correlated with reproductive success (Grabowska-Zhang et al., 2012, 2011). An additional advantage between familiar neighbors may be more spatial tolerance when no competitive behavior is displayed (Hyman and Hughes, 2006). Our data does not provide information on the vocal activity and the behavior displayed during the forays, but it is possible that the extraterritorial forays we documented here were thus not very risky in terms of encountering aggression. In contrast to what we predicted, individuals of different personality types may therefore not show different foraying activity.

Our results thus suggest that the higher social network connectivity of territorial fast-exploring males reported earlier for the same study population (Snijders et al., 2014) does not result from forays into the nest box area of neighboring territories. Instead, such higher connectivity may result from other encounters, for example at foraging sites away from the core territories we monitored (e.g. Aplin et al., 2013). Encounters outside core territories may also lead extra-pair copulations: Patrick et al. (2012) found bolder, faster exploring male great tits to be more likely to father extra-pair offspring, but also more likely to get cuckolded, suggesting that bolder 
males may foray off-territory more often and as a result have more extra-pair encounters, but also mate guard their mate less. In social lizards (Egernia whitii) more aggressive females were more likely to have extra-pair offspring (While et al., 2009), suggesting that female personality also affects the pursuit of extra-pair copulations. However, we here did not observe that faster exploring males or females intrude into the area around boxes of other breeding pairs more often and previously did not find any association between foraying activity and occurrence of extra-pair offspring (Chapter 2). This suggests that the main function of the extra-territorial forays documented here was unlikely to gain extra-pair copulations and that extra-pair behavior in great tits probably does not take place in the vicinity of nest boxes. Faster exploring males may leave their territory more often to visit not monitored 'neutral areas' and as a result achieve more extra-pair encounters.

However, we cannot rule out that fast and slow exploring individuals may have ended up showing similar foraying activity, but for different reasons. Fast exploring individuals might engage in off-territory foraying as a by-product from their general movement behavior or following social motivations, since a higher exploratory score is related to patterns of space use (van Overveld and Matthysen, 2010), but also aggressiveness (Drent et al., 1996) and social connectivity (Aplin et al., 2013; Snijders et al., 2014) in great tits. However, Snijders et al. (2014) did not find evidence that more social encounters are associated with movement speed. Slow exploring individuals, on the other hand, may engage in off-territory foraying to gather more (public) information, for example on reproductive success (Morinay et al., 2020), since such individuals were previously shown to make more use of social information (Kurvers et al., 2010; Smit and van Oers, 2019) and be more prone to sample environmental information repeatedly (van Overveld and Matthysen, 2013).

Our results suggest that partner personality may be important for only male spatial behavior, as we found no association between partner personality and female foraying activity. Male foraying behavior was associated with the combination of both a males' own and their mate's exploratory score. When paired with a slow exploring female, faster exploring males made more forays and tended to visit more different areas, but when paired with a fast exploring female, faster and slower exploring males did not differ from each other in their foraying behavior. As foraying behavior of females themselves here was not associated with their own exploratory score (see above), males do not seem to have simply adjusted their foraying behavior in response to personality-dependent foraying activities of females. However, if slow 
exploring females have an overall lower encounter rate with other individuals (Aplin et al., 2013), the risk of cuckoldry might be less for the males paired with them and fast exploring males may respond by investing less in mate guarding and foray more often independently offterritory. However, previous studies found that males paired with faster exploring females were more likely to sire extra-pair offspring (Patrick et al., 2012) and pairs of extreme fast or slow males and females have higher rates of extra-pair paternity in their broods, in contrast to disassortative pairs (van Oers et al., 2008). Investigating whether or not pair-personality is associated with the number of forays undertaken by a pair together or whether foraying activity is associated with pair personality differently in various breeding stages of the female (for example during the fertile time vs. the non-fertile time) may help explain how males of different personality types adjust their own foraying behavior in response to the females' activity.

Taken together, our results indicate that an individual's own personality in combination with partner personality may play an important role in off-territory movements for males, but not females. Moreover, individual exploratory behavior alone was not associated with an individual's tendency to foray off-territory into the vicinity of the nest box of another breeding pair, indicating that these movements may not be responsible for personality-dependent social network positions or personality-dependent extra-pair paternity patterns in territorial animals. These findings raise intriguing questions on the role of personality in animal movements and social relations as they add a new level of complexity to an individual's spatial decision making with impacts on the behavioral mechanisms underlying social network structures. Moreover, off-territory forays might take place primarily within familiar neighborhoods and may thus reveal an important mechanism facilitating neighborhood nest-defense and familiarity-related reproductive success in territorial animals. 


\section{Acknowledgments}

We would like to thank all the students that were involved in the fieldwork at Westerheide during spring 2016 or 2017: Mara Ruiz Minano, Maeliss Hoarau, Laura Lute, Tomas Tuvillo, Hongye Zhang, Rahman Rasyidi, Lisanne Meulendijks, Bas van Boomgaard and Sander Bik. Special thanks also to Piet de Goede, Christa Mateman, Martijn van der Sluijs, Lydia Nieuwe Weme and all the animal care takers at NIOO-KNAW for their help throughout the project. This study was permitted by the Dutch legal entity Dier Experimenten Comissie (DEC) no. NIOO-10.05 to $\mathrm{MN}$ and $\mathrm{KvO}$ and no. NIOO 12.02 to KvO. The project was funded by an NWO-ALW open competition grant (824.15.012) to MN. 
Supplement to chapter 5 


\section{Model tables}

Table S1: Number of forays per day and characteristics of foraying individual. Table lists all factors included in a zero-inflated negative binomial GLMM with log link function. The dependent variable was the number of forays per day per individual $(N=4267$ observations, 71 individuals). Individual ID was included as random factor (var $\pm s d: 16.31 \pm 4.04)$. Using backward elimination, the estimate and standard error of the last model in which a factor was included are given. The test statistic $\left(\chi^{2}\right)$, degrees of freedom ( $\left.d f\right)$ and significance ( $p$ value) given were determined using likelihood-ratio tests (LRT). The factors year, distance (average distance of the focal individual's nest box location to other nest boxes with receivers) and relative lay date of the focal individual's brood were included in the last model, independent of significance.

\begin{tabular}{|c|c|c|c|c|c|}
\hline & Estimate & se & $\chi^{2}$ & df & P value \\
\hline Intercept & 0.79 & 0.49 & - & - & - \\
\hline Sex (male) & -2.42 & 0.19 & 110.45 & 1 & $<0.001$ \\
\hline Year (2017) & 4.78 & 0.24 & - & - & - \\
\hline Distance (scaled) & -4.78 & 0.30 & - & - & - \\
\hline Relative lay date (scaled) & 0.06 & 0.14 & - & - & - \\
\hline \multicolumn{6}{|l|}{ Dropped terms } \\
\hline Exploratory score $*$ sex (male) & -0.52 & 0.61 & 0.77 & 1 & 0.38 \\
\hline Exploratory score & -0.14 & 0.47 & 0.10 & 1 & 0.77 \\
\hline
\end{tabular}

Table S2: Number of nest box areas visited per day and characteristics of foraying individual. Table lists all factors included in a poisson GLMM with log link function. The dependent variable was the number of nest box areas visited per day per individual ( $N=4267$ observations, 71 individuals). Individual ID was included as random factor (var $\pm s d: 1.62 \pm 1.27$ ). Using backward elimination, the estimate and standard error of the last model in which a factor was included are given. The test statistic $\left(\chi^{2}\right)$, degrees of freedom ( $\left.d f\right)$ and significance $(p$ value) given were determined using likelihood-ratio tests (LRT). The factors year, distance (average distance of the focal individual's nest box location to other nest boxes with receivers) and relative lay date of the focal individual's brood were included in the last model, independent of significance.

\begin{tabular}{|c|c|c|c|c|c|}
\hline & Estimate & se & $x^{2}$ & df & P value \\
\hline Intercept & -0.79 & 0.17 & - & - & - \\
\hline Sex (male) & -0.41 & 0.11 & 14.57 & 1 & $<0.001$ \\
\hline Year (2017) & 1.53 & 0.13 & - & - & - \\
\hline Distance (scaled) & -1.32 & 0.14 & - & - & - \\
\hline Relative lay date (scaled) & 0.39 & 0.07 & - & - & - \\
\hline \multicolumn{6}{|l|}{ Dropped terms } \\
\hline Exploratory score $*$ sex (male) & -0.02 & 0.20 & 0.007 & 1 & 0.93 \\
\hline Exploratory score & -0.02 & 0.02 & 1.36 & 1 & 0.24 \\
\hline
\end{tabular}






\section{CHAPTER 6 \\ General discussion}



Bird song has served as a prime example of a sexually selected ornament for many decades. Its general role in male-male competition and female attraction is well established (Catchpole and Slater, 2008; Searcy and Yasukawa, 1996). However, many aspects of how receivers respond to this signal are still not well understood. The conspicuous peak in singing activity around dawn (dawn song) that occurs in so many bird species around the world (Staicer et al., 1996), for example, is thought to play a significant role in territory defense, resolving territorial disputes and attracting females for extra-pair copulations. However, we know little about the actual spatial behavior of potential receivers, both females and males, around this time compared to the rest of the day. Whether intrusion rates by males and foraying activity by females are particularly high at dawn and whether these movements are related to the dawn song characteristics of territorial males is thus still not well understood (Gil and Llusia, 2020; Staicer et al., 1996). Moreover, bird song is highly interactive and dynamic, with some of its information conveyed through specific matching and timing strategies during male-male vocal interactions (Burt and Vehrencamp, 2005; Catchpole and Slater, 2008; Naguib, 2005; Todt and Naguib, 2000). How exactly males use these singing strategies and what they signal about the respective individuals is still debated (Helfer and Osiejuk, 2015; Naguib and Mennill, 2010; Searcy and Beecher, 2011, 2009). In this thesis, I, therefore, used a combination of automated radio-tracking of multiple potential receivers (Mennill et al., 2012b; Snijders et al., 2017b), recorder arrays (Mennill et al., 2012a) and interactive playbacks (Dabelsteen, 1992; King, 2015 ) to study the role of male song in the spatial behavior and reproductive decision making in great tits. In Chapter 2-4 in particular, I tried to answer the following questions:

- When do females and males foray into other territories, and are these visits related to the resident male's ornaments, particularly dawn singing behavior, and extra-pair paternity?

- Does singing behavior during song contests reflect relative differences between males and do eavesdropping males and females change their foraying behavior or reproductive investment based on such information?

Although forays into other territories have often been proposed to serve the search of extra-pair mates (Kempenaers et al., 1992; Kleven et al., 2006; Schlicht et al., 2015), they also could have multiple other purposes, such as the search for additional food sources or information gathering, (Doligez et al., 2002; Messier, 1985) and might come with substantial costs, like an increased predation risk or stress level (Metzgar, 1967; Young and Monfort, 2009). How individuals 
trade-off these costs with the potential benefits can vary based on their propensity to take risks and consistent individual behavioral differences (personality) (Sih et al., 2004; van Oers et al., 2005; van Oers and Naguib, 2013) may thus be an important factor influencing which animals foray how often, independent of male singing behavior. In Chapter 5, I aimed to examine the link between foraying activity and personality. Here I will synthesize and discuss my findings and outline knowledge gaps that would be interesting to address in future studies and analyses to better understand this complex communication system.

\section{When are females and males foraying?}

To understand how animals use information conveyed by certain signals it is important to have knowledge about the behavior of different potential receivers at different times, which can be difficult to obtain as some receiver movements can be cryptic and difficult to follow. In Chapter

2, Chapter 3 and Chapter 5, I used an automated radio-tracking system that allows to monitor the movements of multiple receivers simultaneously, focusing on foraying activity throughout a breeding season in Chapter 2, short term changes in foraying activity after simulated territorial intrusions in Chapter 3 and variation in foraying activity connected to personality differences between individuals in Chapter 5. In Chapter 2, I observed that both female and male great tits forayed into other territories throughout the entire day and breeding season. The foraying activity of both sexes peaked during the nestling phase and later in the morning and afternoon rather than early in the morning (including dawn). This suggests that the intrusion pressure from other males is not very high around dawn, an important assumption for the territory defense and social dynamics hypotheses (Gil and Llusia, 2020; Kacelnik and Krebs, 1983; Staicer et al., 1996). However, male forays were longest early in the morning: these extended forays might stem from territory neighbors getting into the vicinity of another nest box when resolving territory boundary disputes at dawn. It is important to note though, that visits to territories were in general very short, including the longer ones early in the morning. Moreover, the data analyzed here only included males breeding and thus holding a territory in the respective season, and any intrusions from "floater" individuals still trying to occupy territories would thus not be observed. Dawn might still be an important time for these individuals to prospect territory occupation (Amrhein et al., 2004a; Roth et al., 2009). Detailed tracking during dawn in a smaller neighborhood could provide information where exactly males are located and singing during the early morning (Foote et al., 2008b; Snijders et al., 2014). 
Earlier studies have proposed that dawn may be critical for female foraying and extra-pair copulations (Greives et al., 2015; Halfwerk et al., 2011; Kempenaers et al., 2010). Female foraying activity here was not particularly high around dawn, as reported for some other species (Double and Cockburn, 2000). In contrast, females often forayed during the entire day, in the later morning and afternoon. However, female forays were the longest early in the morning, so females may still assess and encounter potential extra-pair mates during this time, even though forays into other territories were overall short, including the ones early in the morning. In some species, female extra-pair copulations were observed during brief visits to close-by, neighboring territories (Kempenaers et al., 1992; Sheldon, 1994; Smith, 1988). As dawn song is a long-range signal, females may be able to hear and assess neighbors without leaving their territory and potentially even their nest box (Blumenrath et al., 2004), depending on the spacing of individual territories (Blumenrath and Dabelsteen, 2004). In such cases, a low number of forays, or close approaches to the nest box of another male as measured here, may not indicate a low assessment of males (Otter and Ratcliffe, 2005), and the few forays made in the early morning may already represent actual choices. However, the early morning time window in this analysis did not only strictly include the time of dawn but also sometime just after sunrise. Thus, for both males and females, it is also possible that forays in the early morning were forays made in the search for food after the night.

In contrast to previous studies that observed a peak for foraying during the fertile period of females (Neudorf et al., 1997; Pedersen et al., 2006; Ward et al., 2014), and specifically during the few days just before laying (Double and Cockburn, 2000), I did not observe this pattern in Chapter 2. Female great tits also forayed quite often during the fertile days before egg-laying, as well as the egg-laying days, which would be expected if they are foraying to seek extra-pair copulations, but the peak of foraying occurred when females are not fertile anymore, during the nestling stage. This stage is a time of high investment for both parents, and they therefore may be foraging during such visits to other territories. Moreover, female foraying activity here did not predict the occurrence of extra-pair paternity. However, since only one copulation may be needed to have extra-pair offspring, this may not necessarily mean that female foraying is not related to extra-pair mating.

Other studies reported that male off-territory movements decreased during the fertile period of mates and suggested that males might invest more in mate-guarding during this time (Akçay et al., 2012; Churchill and Hannon, 2010; Currie et al., 1998; Evans et al., 2008; Ward et al., 
2014). Male foraying activity here did not seem to decrease remarkably when their mate was fertile. However, it is possible that some forays undertaken during the female's fertile period were joint forays, where males followed their mate on their forays during the fertile period in an attempt to mate guard. Investigating whether pairs foray to the same places simultaneously and whether the number of such joint forays changes over the breeding season may give a more accurate picture of the potential mate-guarding effort of males. In contrast to findings in other species (Kleven et al., 2006; Schlicht et al., 2015), neither the number of forays made by the resident male nor the number of visits received by other males predicted the occurrence of extra-pair offspring in a particular brood. Overall, although both males and females could encounter potential extra-pair mates when foraying, the timing of forays here does not suggest that great tits undertake these movements mainly to search for extra-pair copulations. Instead, visits to other territories seem common throughout the day and season and probably serve multiple purposes, including foraging or information gathering. Supplementing certain individuals with food and subsequently observing how it affects their foraying activity, as done for Northern cardinals (Cardinalis cardinalis) (Humbird and Neudorf, 2008), might give additional insights in why great tits visit other territories.

\section{Are forays linked to male ornaments and extra-pair paternity?}

Previous work suggested that females seek out more elaborately ornamented males when foraying off-territory while males might use acoustic or visual signals to assess the fighting ability or dominance of other males and decide where there is a better chance to cuckold the territory owner (Chiver et al., 2008; Kempenaers et al., 1992; Pedersen et al., 2006). In Chapter 2 and Chapter 3 I investigated the link between male song and plumage traits and female and male foraying behavior.

Both yellowness and the size of the black breast stripe are associated with male quality in the great tit (Norris, 1993; Senar et al., 2008). The size of the black stripe particularly may be important in dominance relationships (Järvi and Bakken, 1984; Lemel and Wallin, 1993; but see Wilson, 1992) and mate choice (Norris, 1990). Although plumage ornaments seem to play an essential role in guiding extra-territorial forays in both males and females in other species (Pedersen et al., 2006), the results in Chapter 2 show that there is no link between female or male visits to an area and the respective male's plumage ornaments. Stripe size may be most important in settling dominance relationships with unknown individuals and in situations of very high motivation, for example under strong food competition, (Lemel and Wallin, 1993), 
and thus might not have influenced foraying rates into territories within a known, already established neighborhood (Temeles, 1994). Moreover, plumage ornaments, as visual signals, require a closer, potentially risky approach to be seen and assessed and may have less of an influence on off-territory movements than song, which can be heard from further away. Yellowness or breast stripe size may thus play a more important role in close encounters, for example when settling disputes at common feeding places or once individuals have already entered the territory of another pair. However, there was no association between the duration of visits to territories and a resident's plumage ornaments, suggesting that a larger breast stripe for example did not lead to intruding males staying for a shorter time. However, the tracking data here does not provide information about whether visiting individuals actually encountered the resident male: to study whether residents with certain plumage traits are more successful in keeping intruders away or attracting females, more detailed data on actual close encounters would be necessary.

In Chapter 2 I show that how a male sang at dawn predicts how often his territory is visited by females and males not only at dawn but in general, throughout the day and breeding season. Thereby, a more elaborate dawn song did not seem to act as a deterrent or "keep out signal" to other males, an important prediction following the standard function in territory defense of male song (Catchpole and Slater, 2008; Kacelnik and Krebs, 1983). Males here forayed more often and for a longer time to nest box areas of resident males with a high song rate and proportion of time spent singing during the dawn song. Since the foraying data only includes breeding males and, therefore, territory holders, it is possible that, within an established neighborhood where males hear each other singing every day, a high singing activity may be assed differently by other males. Males might be attracted to territories of such males for various reasons, for example, to forage as singing activity can indicate food availability (Ritschard and Brumm, 2012) or they may visit such areas more often to gather information on habitat quality or breeding success for future breeding attempts (Doligez et al., 2004b, 1999). However, male visits to nest box areas of residents singing longer songs during their dawn song were shorter and also tended to be shorter when residents had a large repertoire size and an early and long dawn song. Krebs et al. (1978) showed that male great tits with a more extensive repertoire are more successful in keeping intruders out, and mean song duration is positively correlated with dominance in this species (Lambrechts and Dhondt, 1986). These aspects of male dawn singing may thus still act as a deterrent to other males, even though males did not visit territories of males with longer songs and larger repertoires less often. Rather than keeping other males 
entirely away, they may lead to shorter intrusions and thus ease the settlement of boundary disputes as other males do not want to risk closer encounters.

Dawn song has been proposed to serve the attraction of extra-pair mates as dawn might be an essential time for extra-pair mating (Double and Cockburn, 2000; Halfwerk et al., 2011; Kempenaers et al., 2010, 1997; Poesel et al., 2006), presumably because detection by the social mate is less likely during that time and females would not risk for example aggression from or loss of parental care by their mate (Westneat and Stewart, 2003). In the great tit specifically, female emergence time from the nest box (Halfwerk et al., 2011), male sleeping duration (Greives et al., 2015) and male dawn song characteristics (Halfwerk et al., 2011; Kempenaers et al., 2010) have been linked to the occurrence of extra-pair offspring. In contrast to these suggestions, the observations on female foraying behavior in Chapter 2 show that females visited nest box areas less often when resident males had a larger dawn repertoire, started their dawn song earlier and sung for longer at dawn. As discussed above, females also did not foray particularly often during dawn. Although these results do not exclude the possibility of extrapair copulations taking place during forays and some of them happening in the early morning, they do not suggest that sneaking out around dawn to seek extra-pair copulations is a major reason for female foraying. Similarly to elaborate dawn song, higher levels of signal matching and overlapping during singing interactions between males throughout the day have been suggested to influence female foraying behavior and (extra-pair) mate assessment (Mennill et al., 2003, 2002; Otter et al., 1999). In great tits, females forayed more often to other territories after hearing their mate being overlapped at high levels by a simulated intruder, possibly to assess potential extra-pair mates (Otter et al., 1999), but these forays did not result in different mating patterns (Otter et al., 2001). In Chapter 3, I also observed no difference in the foraying behavior of females with and without extra-pair offspring after their mates were exposed to an overlapping, more threatening intruder in a simulated territory intrusion. Together, the results in Chapter 2 and Chapter 3 thus do not suggest that females seek out more elaborately ornamented males when foraying off-territory.

Several studies in great tits found no difference between extra-pair mates and social mates in multiple measures such as age, body size, the width of the breast stripe, survival, likelihood to be divorced, likelihood to be cuckolded and how many of their offspring were recruited to the local population (Krokene et al., 1998; Lubjuhn et al., 1999; Strohbach et al., 1998). Thus, gaining indirect benefits may not be the reason for extra-pair mating in females in this species. 
Benefits of extra-pair mating for females are still debated in general (reviewed in Brouwer and Griffith, 2019), and overall consistent evidence for indirect benefits seems to be lacking (Akçay and Roughgarden, 2007; Forstmeier et al., 2014; Hsu et al., 2015). In the great tit, variation in extra-pair paternity seems to be linked to personality traits of individuals and their mates (Patrick et al., 2012; van Oers et al., 2008). Personality may influence an individual's propensity to foray off-territory (and encounter potential extra-pair mates) in the first place. Bolder, fasterexploring males were more likely to father extra-pair offspring and to get cuckolded (Patrick et al., 2012), potentially because they foray off-territory more often and as a result have more extra-pair encounters. However, not only an individual's personality but also their partner's may be necessary for explaining foraying behavior, since males paired with faster-exploring females were more likely to sire extra-pair offspring (Patrick et al., 2012). Moreover, pairs of extreme fast or slow males and females have higher rates of extra-pair paternity in their broods than disassortative pairs (van Oers et al., 2008). In Chapter 5, I therefore explored whether there is a relationship between individual personality, partner personality, and their combination and foraying activity. The results do not imply a simple relationship with bolder, fasterexploring individuals foraying more often. Thus, the previously reported higher social connectivity of faster-exploring territorial individuals (Snijders et al., 2014) or the higher likelihood of bolder males to sire extra-pair offspring (Patrick et al., 2012) may not be a result of these individuals intruding more often into other territories and therefore having more encounters with other birds, as individual personality was not associated with foraying behavior. Together with the observation from Chapter 2, where foraying activity was not associated with the occurrence of extra-pair paternity, these findings suggest that seeking extrapair copulations is not a primary function of these territory intrusions and that extra-pair behavior in great tits most likely does not take place in the vicinity of nest boxes. Monitoring areas between territories and nest boxes would allow us to investigate how faster-exploring males come about higher extra-pair paternity rates (and a higher social connectivity) (Patrick et al., 2012; Snijders et al., 2014), as they might encounter potential copulation partners there. The results in Chapter 5 further show that male foraying behavior is associated with both a male's own and his partner's personality: faster-exploring males made more forays and tended to visit more different areas when they were paired with a slow exploring female. However, when paired with a fast exploring female, faster and slower exploring males did not differ in their foraying behavior, suggesting that male foraying behavior may be influenced by both his own and his partners' personality. To understand how males of different personality types adjust their foraying behavior in response to the females' activity, it would be interesting to 
study whether pairs often foray together and whether the time spent together off-territory is influenced by pair personality. Moreover, the results from Chapter 2 and Chapter 5 together suggest that to gain insights into the behavioral interaction between male, female and extra-pair male we need to monitor where both members of a pair meet other individuals (with, for example, proximity loggers) in areas away from the vicinity of breeding boxes. Monitoring such encounters will also help us to understand how the higher social connectivity of more exploratory personality types develops (Snijders et al., 2014).

The observation that dawn song was associated with female foraying activity, but in the opposite way than expected, is interesting and suggests that females might also use the information conveyed in this signal in different ways than previously assumed. Females might, for example, use the information contained in male song to avoid more aggressive males (Bierbach et al., 2013; Ophir and Galef, 2003; Qvarnström and Forsgren, 1998) when foraying since elaborate song traits are often associated with male territory tenure, dominance, and willingness to escalate an interaction (Krebs et al., 1978; Otter et al., 1997; Vehrencamp, 2001). Together, these results suggest that the importance of the two proposed functions of dawn song in interactions with other individuals, mate attraction and territory defense (Gil and Llusia, 2020; Kacelnik and Krebs, 1983; Staicer et al., 1996), may depend on the time frame in focus. Males and females seem to make use of the information signaled by dawn song in a different way than expected when looking at overall movements throughout the breeding season. Moreover, song is a multifaceted behavior (Gil and Gahr, 2002) with different characteristics of it potentially being used differently by various receivers. Thus, which information conveyed by dawn song is most relevant to which audience and how it is used may depend on the time window considered. Similarly, different dawn song characteristics might not have the same relative importance at different breeding season stages. Therefore, it would be interesting to examine whether the relationship between male dawn singing and female and male movements and the specific characteristics of the dawn song, that are important changes between different stages of the breeding season.

\section{What information is conveyed by singing patterns in male-male vocal interactions?}

Song type matching and song overlapping have been proposed to be signals of aggressiveness or willingness to escalate an interaction and asymmetries in the usage of such singing patterns in interaction to reflect relative differences between rivals that can signal important information to potential eavesdroppers (Catchpole and Slater, 2008; Todt and Naguib, 2000). Indeed, 
studies in several species have shown that males respond more strongly to intruders that type match and overlap their song and both males and females seem to eavesdrop on singing interactions and change their behavior ins subsequent territorial encounters (Hall et al., 2006; Mennill and Ratcliffe, 2004; Naguib et al., 2004, 1999; Naguib and Todt, 1997; Peake et al., 2002, 2001) or reproductive decisions (Amy et al., 2008; Garcia-Fernandez et al., 2010; Mennill et al., 2002) depending on male's overlapping or matching behavior.

In Chapter 3, I did not observe any evidence for a stronger response to a more threatening intruder (that was overlapping the resident males' song and also singing on the territory for longer) compared to a less threatening intruder. Moreover, I did not observe any change in the spatial behavior or reproductive investment of the resident male's mate nor the spatial behavior of male and female neighbors that could have indicated eavesdropping. In Chapter 4, I did not find any relationship between the level of song matching and song overlapping a male used in an interaction and the relative difference between him and his opponent in several characteristics that might be important for eavesdroppers to assess males in territorial disputes or reproductive decision making. Overall, the results here do thus not provide any evidence in support for overlapping being a signal of aggressive intent or both matching or overlapping signaling relevant information to eavesdropping individuals in the great tit.

There are several possible explanations as to why there was no evidence for eavesdropping on the interaction with a simulated aggressive intruder in Chapter 3. Firstly, playback subjects did not react more strongly to simulated threatening than less threatening intruders, maybe indicating that they did not perceive overlapping and singing on the territory for longer as more threatening in the first place. It is possible that not only overlapping itself but also the level of overlapping used is crucial in conveying any aggressive intent with this singing pattern (Naguib and Kipper, 2006). However, the level of overlapping used in the playback in Chapter 3 was similar to what a previous study (Amy et al., 2010) in the same population used that observed a stronger reaction to an overlapping intruder. Although often interpreted as a signal of aggressive intent, the signal function of overlapping has been subject to debate. Overlapping has also been proposed to just occur by chance whenever signaling animals share an acoustic space and to be an incidental interference that animals actively try to avoid or even to act as a submissive, rather than aggressive, signal (reviewed in Helfer and Osiejuk, 2015; Naguib and Mennill, 2010; Searcy and Beecher, 2011, 2009). 
However, females might not have changed their spatial or reproductive behavior in response to their mate being confronted by the more challenging intruder because they have long-term experience with their mate and information on many interactions. Thus, they might not re-assess mate quality and change reproductive decisions based on a single interaction. Previous studies that reported that females differentially allocate resources to eggs based on male quality signaled by song, have detected these changes in allocation mostly in androgen concentrations(Gil et al., 2004, 1999a; Tanvez et al., 2004). Garcia-Fernandez et al. (2010) reported a change in the yolk to egg ratio of females that were exposed to song they previously had heard as being the overlapping song in a song contest, although other measures of resource investment, including egg weight, were not affected (Garcia-Fernandez et al., 2010). As I only quantified female investment in terms of egg weight and provisioning behavior, females might have changed their investment, but these changes were not detected. Similarly, neighbors might have reacted in ways that were not considered, for example, vocally through a higher song output (Fitzsimmons et al., 2008a; Naguib et al., 2004). It would thus be interesting to challenge resident males multiple times with simulated territory intrusions and monitor female and neighbor responses in more detail, to examine possible more fine-tuned ways in which great tits could use information gathered through eavesdropping on male-male contests.

In Chapter 4, I did not find any evidence that song type matching or song overlapping during natural singing interactions between great tit males reflect relative differences between them in the individual characteristics considered. One possible explanation might be that the interactions analyzed took place between established neighbors. Singing patterns like matching and overlapping might be especially important in interactions with unknown individuals, as simulated by most playback studies (Naguib, 2005; Searcy and Beecher, 2009). Often territorial animals react more aggressively to unfamiliar strangers than to familiar neighbors (reviewed in Temeles, 1994), and in songbirds, the song of known individuals is usually perceived as less threatening (reviewed in Stoddard, 1996). Moreover, the timing of the interactions might play an important role, as at least song type matching in great tits has been reported to occur more often in the winter, before or at the time of territory establishment, when boundary disputes are likely more heated and males more motivated during song contests (Krebs et al., 1981). Krebs et al. (1981) described high proportions of interactions between males in which song type matching occurred in the beginning of January, that then decreased towards mid-February. Therefore, it would be necessary to monitor singing interactions early in the year during territory occupation to see whether the use of matching and overlapping during that time reflects 
individual differences between males and whether males that occupy territories in preferred habitat are indeed matching and overlapping more often than others. However, many of the experiments that provided evidence for a role of matching and overlapping in male-male interactions in the great tit have taken place much later (Dabelsteen et al., 1996; Langemann et al., 2000; McGregor et al., 1992; Otter et al., 1999; Peake et al., 2002, 2001), during early spring, and therefore during approximately the same time frame as considered in Chapter 4. Thus, these singing patterns would still be expected to signal relevant information at this time of year. It would be interesting to see whether the level of matching and overlapping is repeatable within an individual or whether the use of these singing patterns and the information signaled through them changes over time. Signal matching of another individual, for example, could also be used to "address" a specific receiver, as has been observed in Bottlenose dolphins (Tursiops truncatus) (King et al., 2014). Alternatively, high-performance singers could benefit from matching others not because matching is an aggressive signal per se, but because singing the same song type as a rival would allow eavesdroppers to more easily compare "how well it is sung”, i.e., how closely both males approach physiological performance limits (Logue and Forstmeier, 2008).

\section{Concluding remarks}

In this thesis I combined automated radio-tracking of both females and males throughout the breeding season with acoustic (array-) recordings, interactive playback experiments, microsatellite analysis, plumage measures, RFID tag and video monitoring to quantify 1) between individual variation in dawn singing as well as singing patterns during interactions throughout the day 2) receiver spatial behavior, extra-pair mating patterns and reproductive investment. Using this integrated approach, I showed that territories of males with elaborate dawn song overall are not visited more often by females and less often by males, an important prediction following the common interpretation of dawn song as a signal in male-male competition and female attraction. Moreover, intrusion pressure by males and visitation rates by females were not particularly high at dawn. This finding highlights that singing behavior, the information conveyed by it and how receivers make use of this information may change depending on the time window considered. To study whether and how the importance of dawn song for male and female receivers changes over time and gain a more detailed insight in how males and females might be using this signal differently it would thus be interesting to remove males temporally at varying stages during the season at dawn. Replacing their dawn song with playback while manipulating different song characteristics, in combination with tracking 
surrounding receivers may allow us to disentangle how males and females are using this signal (and different aspects of it) differently.

Using this long-term tracking data, I also showed that female visits to other territories are negatively correlated with elaborate song by males, not associated with elaborate plumage traits and do not predict extra-pair paternity patterns. Moreover, forays are not timed in a way (for example occurring particularly often during times of fertility) that suggests that females are using these forays particularly to seek extra-pair copulations with particular males, which would be expected if females were to gain indirect benefits from extra-pair mating. My results on female behavior in the great tit thus add to a growing body of literature that questions whether females seek indirect benefits through extra-pair mating (Akçay and Roughgarden, 2007; Forstmeier et al., 2014; Hsu et al., 2015). Rather, the results here support the findings of previous studies (Patrick et al., 2012; van Oers et al., 2008) that reported that variation in extrapair paternity in this species is connected to consistent behavioral differences (personality) between individuals and their mates that might influence the interplay between female, male and extra-pair mate as I documented here that male spatial behavior is linked to both the own and the mate's exploratory behavior.

Previous work established that the interactive, highly dynamic nature of song (Naguib, 2005; Todt and Naguib, 2000) needs to be considered when trying to understand the role of this signal in both female attraction and male-male competition. Signaling patterns arising during interactions only, and not through solo singing alone, may convey an additional layer of information accessible to receivers (Dabelsteen, 1992; McGregor et al., 2000; Todt and Naguib, 2000). Despite evidence from previous studies that asymmetries in singing strategies such as song matching and overlapping in male song interactions provide important information for the assessment of rivals and mates (Garcia-Fernandez et al., 2010; Mennill et al., 2002; Mennill and Ratcliffe, 2004; Otter et al., 1999; Peake et al., 2002), the combination of an interactive playback experiment, tracking receiver movements and monitoring naturally occurring singing interactions with a recorder array that I employed here did not lead to supporting evidence. These findings suggest that the importance of singing patterns such as type matching and overlapping may be highly time and context specific and receiver responses might be subtle and not easily followed in the wild. My results thus emphasize the need for further research to pinpoint which aspects of male singing interactions specifically are assessed and whether, when and how males and females use any information gathered by eavesdropping on such 
interactions. Using animal borne devices, which allow for vocalizations to be recorded directly from the sound-producing animal and thus assigned unequivocally while retaining temporal precision may be a way to examine such vocal interactions in more detail (Anisimov et al., 2014; Gill et al., 2016). In that way, interactions could be investigated with individual-level resolution when males are close together, for example at territory boundaries during dawn and during the day, as well as when they interact from far away. In conclusion, my research emphasizes the view that bird song is a multifaceted signal (Gil and Gahr, 2002) that can be directed at and heard by many receivers simultaneously (McGregor, 1993; McGregor and Peake, 2000) and that to understand both proximate and ultimate factors shaping this communication system, it is fundamental to obtain reliable information on the behavior of participating individuals and their response on different levels and at different times. 


\section{References}

Abramoff, M.D., Magalhães, P.J., Ram, S.J., 2004. Image processing with imageJ.

Biophotonics Int. 11, 36-41.

Akçay, Ç., Tom, M.E., Campbell, S.E., Beecher, M.D., 2013. Song type matching is an honest early threat signal in a hierarchical animal communication system. Proc. R. Soc. B Biol. Sci. 280, 20122517.

Akçay, Ç.Ç., Searcy, W.A., Campbell, S.E., Reed, V.A., Templeton, C.N., Hardwick, K.M., Beecher, M.D., 2012. Who initiates extrapair mating in song sparrows? Behav. Ecol. 23, 44-50.

Akçay, E., Roughgarden, J., 2007. Extra-pair paternity in birds: Review of the genetic benefits. Evol. Ecol. Res. 9, 855-868.

Amrhein, V., Erne, N., 2006. Dawn singing reflects past territorial challenges in the winter wren. Anim. Behav. 71, 1075-1080.

Amrhein, V., Johannessen, L.E., Kristiansen, L., Slagsvold, T., 2008. Reproductive strategy and singing activity: blue tit and great tit compared. Behav. Ecol. Sociobiol. 62, 16331641.

Amrhein, V., Kunc, H.P., Naguib, M., 2004a. Non-territorial nightingales prospect territories during the dawn chorus. Proc. R. Soc. B Biol. Sci. 271, S167-S169.

Amrhein, V., Kunc, H.P., Naguib, M., 2004b. Seasonal patterns of singing activity vary with time of day in the nightingale (Luscinia megarhynchos). Auk 121, 110-117.

Amrhein, V., Lerch, S., 2010. Differential effects of moving versus stationary territorial intruders on territory defence in a songbird. J. Anim. Ecol. 79, 82-87.

Amy, M., Monbureau, M., Durand, C., Gomez, D., Théry, M., Leboucher, G., 2008. Female canary mate preferences: differential use of information from two types of male-male interaction. Anim. Behav. 76, 971-982.

Amy, M., Sprau, P., De Goede, P., Naguib, M., 2010. Effects of personality on territory defence in communication networks: a playback experiment with radio-tagged great tits. Proc. R. Soc. B Biol. Sci. 277, 3685-3692.

Andersson, M., 1994. Sexual Selection. Princeton University Press, Princeton, New Jersey, New Jersey.

Anisimov, V.N., Herbst, J.A., Abramchuk, A.N., Latanov, A. V., Hahnloser, R.H.R., Vyssotski, A.L., 2014. Reconstruction of vocal interactions in a group of small songbirds. Nat. Methods 11, 1135-1137. 
Aplin, L.M., Farine, D.R., Morand-Ferron, J., Cole, E.F., Cockburn, A., Sheldon, B.C., 2013. Individual personalities predict social behaviour in wild networks of great tits (Parus major). Ecol. Lett. 16, 1365-1372.

Baker, T.M., Wilson, D.R., Mennill, D.J., 2012. Vocal signals predict attack during aggressive interactions in black-capped chickadees. Anim. Behav. 84, 965-974.

Bates, D., Mächler, M., Bolker, B., Walker, S., 2015. Fitting linear mixed-effects models using lme4. J. Stat. Softw. 67, 1-48.

Behr, O., von Helversen, O., Heckel, G., Nagy, M., Voigt, C.C., Mayer, F., 2006. Territorial songs indicate male quality in the sac-winged bat Saccopteryx bilineata (Chiroptera, Emballonuridae). Behav. Ecol. 17, 810-817.

Berglund, A., Bisazza, A., Pilastro, A., 1996. Armaments and ornaments: an evolutionary explanation of traits of dual utility. Biol. J. Linn. Soc. 58, 385-399.

Bierbach, D., Sassmannshausen, V., Streit, B., Arias-Rodriguez, L., Plath, M., 2013. Females prefer males with superior fighting abilities but avoid sexually harassing winners when eavesdropping on male fights. Behav. Ecol. Sociobiol. 67, 675-683.

Bircher, N., Naguib, M., 2020. How songbird females sample male song: communication networks and mate choice., in: Aubin, T., Mathevon, N. (Eds.), Coding Strategies in Vertebrate Acoustic Communication. Springer Verlag, Heidelberg.

Bircher, N., van Oers, K., Hinde, C.A., Naguib, M., 2020. Extraterritorial forays by great tits are associated with dawn song in unexpected ways. Behav. Ecol. 31, 873-883.

Birkhead, R., 1992. Numbers and size of sperm storage tubules and the duration of sperm storage in birds: a comparative study. Biol. J. Linn. Soc. 45, 363-372.

Birkhead, T.R., Cunningham, E.J.A., Cheng, K.M., 1996. The insemination window provides a distorted view of sperm competition in birds. Proc. R. Soc. B Biol. Sci. 263, 11871192.

Birkhead, T.R., Møller, A.P., 1992. Sperm competition in birds: evolutionary causes and consequences. Academic Press, London.

Bischoff, L.L., Tschirren, B., Richner, H., 2009. Long-term effects of early parasite exposure on song duration and singing strategy in great tits. Behav. Ecol. 20, 265-270.

Blumenrath, S.H., Dabelsteen, T., 2004. Degradation of great tit (Parus major) song before and after foliation: implications for vocal communication in a deciduous forest. Behaviour 141, 935-958.

Blumenrath, S.H., Dabelsteen, T., Pedersen, S.B., 2004. Being inside nest boxes: does it complicate the receiving conditions for great tit Parus major females? Bioacoustics 14, 
209-223.

Bolsinger, J.S., 2000. Use of two song categories by golden-cheecked warblers. Condor 102, $539-552$.

Boon, A.K., Réale, D., Boutin, S., 2008. Personality, habitat use, and their consequences for survival in North American red squirrels Tamiasciurus hudsonicus. Oikos 117, 13211328.

Both, C., Dingemanse, N.J., Drent, P.J., Tinbergen, J.M., 2005. Pairs of extreme avian personalities have highest reproductive success. J. Anim. Ecol. 74, 667-674.

Bradbury, J.W., Vehrencamp, S.L., 1998. Principles of Animal Communication, 2nd ed. Sinauer Associates, Inc., Publishers, Sunderland, Massachusetts.

Brooks, M.E., Kristensen, K., van Benthem, K.J., Magnusson, A., Berg, C.W., Nielsen, A., Skaug, H.J., Maechler, M., Bolker, B.M., 2017. glmmTMB balances speed and flexibility among packages for zero-inflated generalized linear mixed modeling. R J. 9, $378-400$.

Brouwer, L., Griffith, S.C., 2019. Extra-pair paternity in birds. Mol. Ecol. 00, 1-19.

Bruinzeel, L.W., van de Pol, M., 2004. Site attachment of floaters predicts success in territory acquisition. Behav. Ecol. 15, 290-296.

Brumm, H., Naguib, M., 2009. Environmental acoustics and the evolution of bird song. Adv. Study Behav. 40, 1-33.

Bruni, A., Foote, J.R., 2014. Dawn singing of Eastern phoebes varies with breeding stage and brood number. Wilson J. Ornithol. 126, 500-507.

Burt, J.M., Vehrencamp, S.L., 2005. Dawn chorus as an interactive communication network, in: McGregor, P.K. (Ed.), Animal Communication Networks. Cambridge University Press, pp. 321-450.

Byers, B.E., 2007. Extrapair paternity in chestnut-sided warblers is correlated with consistent vocal performance. Behav. Ecol. 18, 130-136.

Byers, B.E., Kroodsma, D.E., 2009. Female mate choice and songbird song repertoires. Anim. Behav. 77, 13-22.

Candolin, U., 2003. The use of multiple cues in mate choice. Biol. Rev. 78, 575-595.

Catchpole, C.K., Slater, P.J.B., 2008. Bird song: biological themes and variations, 2nd ed. Cambridge University Press, Cambridge, UK.

Celis-Murillo, A., Schelsky, W., Benson, T.J., Louder, M.I.M., Ward, M.P., 2017. Patterns, correlates, and paternity consequences of extraterritorial foray behavior in the field sparrow (Spizella pusilla): an automated telemetry approach. Behav. Ecol. Sociobiol. 71, 
45.

Chapman, B.B., Hulthén, K., Blomqvist, D.R., Hansson, L.A., Nilsson, J.Å., Brodersen, J., Anders Nilsson, P., Skov, C., Brönmark, C., 2011. To boldly go: Individual differences in boldness influence migratory tendency. Ecol. Lett. 14, 871-876.

Cheney, D.L., Seyfarth, R.M., 1990. The representation of social relations by monkeys. Cognition 37, 167-196.

Cheng, K.M., Burns, J.T., McKinney, F., 1983. Forced copulation in captive mallards III. Sperm competition. Auk 100, 302-310.

Chiver, I., Stutchbury, B.J.M., Morton, E.S., 2008. Do male plumage and song characteristics influence female off-territory forays and paternity in the hooded warbler? Behav. Ecol. Sociobiol. 62, 1981-1990.

Churchill, J.L., Hannon, S.J., 2010. Off-territory movement of male American redstarts (Setophaga ruticilla) in a fragmented agricultural landscape is related to song rate, mating status and access to females. J. Ornithol. 151, 33-44.

Cordero, P.J., Wetton, J.H., Parkin, D.T., 1999. Within-clutch patterns of egg viability and paternity in the house sparrow. J. Avian Biol. 30, 103.

Cote, J., Clobert, J., 2007. Social personalities influence natal dispersal in a lizard. Proc. R. Soc. B Biol. Sci. 274, 383-390.

Cunningham, E.J.A., Russell, A.F., 2000. Egg investment is influenced by male attractiveness in the mallard. Nature 404, 74-77.

Currie, D.R., Burke, T., Whitney, R.L., Thompson, D.B.A., 1998. Male and female behaviour and extra-pair paternity in the wheatear. Anim. Behav. 55, 689-703.

Cuthill, I.C., Macdonald, W.A., 1990. Experimental manipulation of the dawn and dusk chorus in the blackbird Turdus merula. Behav. Ecol. Sociobiol. 26, 209-216.

Dabelsteen, T., 1992. Interactive playback: a finely tuned response., in: McGregor, P.K. (Ed.), Playback Studies of Animal Communication. Plenum Press, New York, pp. 97-109.

Dabelsteen, T., McGregor, P., 1996. Dynamic acoustic communication and interactive playback, in: Kroodsma, D.E., Miller, E.H. (Eds.), Ecology and Evolution of Acoustic Communication in Birds. Cornell University Press, Ithaca, New York, pp. 398-408.

Dabelsteen, T., McGregor, P.K., Shepherd, M., Whittaker, X., Pedersen, S.B., 1996. Is the signal value of overlapping different from that of alternating during matched singing in great tits? J. Avian Biol. 27, 189-194.

Dale, S., Slagsvold, T., 1995. Female contests for nest sites and mates in the pied flycatcher Ficedula hypoleuca. Ethology 99, 209-222. 
Dalziell, A.H., Cockburn, A., 2008. Dawn song in superb fairy-wrens: a bird that seeks extrapair copulations during the dawn chorus. Anim. Behav. 75, 489-500.

Danchin, E., Giraldeau, L.-A., Valone, T.J., Wagner, R.H., 2004. Public information: from nosy neighbors to cultural evolution. Science. 305, 487-491.

Darwin, C., 1871. The descent of man and selection in relation to sex. John Murray, London.

Dingemanse, N.J., Both, C., Drent, P.J., Oers, Kees Van, Van Noordwijk, A.J., Van Oers, K, Van Noordwijk, A.J., 2002. Repeatability and heritability of exploratory behaviour in great tits from the wild. Anim. Behav. 64, 929-938.

Dingemanse, N.J., de Goede, P., 2004. The relation between dominance and exploratory behavior is context-dependent in wild great tits. Behav. Ecol. 15, 1023-1030.

Doligez, B., Danchin, E., Clobert, J., 2002. Public information and breeding habitat selection in a wild bird population. Science. 297, 1168-1170.

Doligez, B., Danchin, E., Clobert, J., Gustafsson, L., 1999. The use of conspecific reproductive success for breeding habitat selection in a non-colonial, hole-nesting species, the collared flycatcher. J. Anim. Ecol. 68, 1193-1206.

Doligez, B., Pärt, T., Danchin, E., 2004a. Prospecting in the collared flycatcher: Gathering public information for future breeding habitat selection? Anim. Behav. 67, 457-466.

Doligez, B., Pärt, T., Danchin, E., Clobert, J., Gustafsson, L., 2004b. Availability and use of public information and conspecific density for settlement decisions in the collared flycatcher. J. Anim. Ecol. 73, 75-87.

Double, M., Cockburn, A., 2000. Pre-dawn infidelity: females control extra-pair mating in superb fairy-wrens. Proc. R. Soc. B-Biological Sci. 267, 465-470.

Doutrelant, C., McGregor, P.K., 2000. Eavesdropping and mate choice in female fighting fish. Behavior 137, 1655-1669.

Drachmann, J., Komdeur, J., Boomsma, J.J., 1997. Copulation behaviour in the linnet Carduelis cannabina and the insemination window hypothesis. J. Avian Biol. 28, 191196.

Drent, P.J., Boon, A., Verbeek, M.E.M., 1996. Exploration, aggressive behaviour and dominance in pair-wise confrontations of juvenile male great tits. Behaviour 133, 945963.

Evans, M.L., Stuchtchbury, B.J.M., Woolfenden, B.E., 2008. Off-territory forays and genetic mating system of the wood thrush (Hylocichla mustelina). Auk 125, 67-75.

Evans, S.R., Hinks, A.E., Wilkin, T.A., Sheldon, B.C., 2010. Age, sex and beauty: methodological dependence of age- and sex-dichromatism in the great tit Parus major. 
Biol. J. Linn. Soc. 101, 777-796.

Falls, J.B., 1988. Does song deter territorial intrusion in white-throated sparrows (Zonotrichia albicollis)? Can. J. Zool. 66, 206-211.

Falls, J.B., Krebs, J.R., McGregor, P.K., 1982. Song matching in the great tit (Parus major): The effect of similarity and familiarity. Anim. Behav. 30, 997-1009.

Figuerola, J., Senar, J.C., 2000. Measurement of plumage badges: an evaluation of methods used in the great tit Parus major. Ibis. 142, 482-484.

Fischer, J., Kitchen, D.M., Seyfarth, R.M., Cheney, D.L., 2004. Baboon loud calls advertise male quality: acoustic features and their relation to rank, age, and exhaustion. Behav. Ecol. Sociobiol. 56, 140-148.

Fitzsimmons, L.P., Foote, J.R., Ratcliffe, L.M., Mennill, D.J., 2008a. Eavesdropping and communication networks revealed through playback and an acoustic location system. Behav. Ecol. 19, 824-829.

Fitzsimmons, L.P., Foote, J.R., Ratcliffe, L.M., Mennill, D.J., 2008b. Frequency matching, overlapping and movement behaviour in diurnal countersinging interactions of blackcapped chickadees. Anim. Behav. 75, 1913-1920.

Foote, J.R., Fitzsimmons, L.P., Mennill, D.J., Ratcliffe, L.M., 2011. Male black-capped chickadees begin dawn chorusing earlier in response to simulated territorial insertions. Anim. Behav. 81, 871-877.

Foote, J.R., Fitzsimmons, L.P., Mennill, D.J., Ratcliffe, L.M., 2010. Black-capped chickadee dawn choruses are interactive communication networks. Behaviour 147, 1219-1248.

Foote, J.R., Fitzsimmons, L.P., Mennill, D.J., Ratcliffe, L.M., 2008a. Male chickadees match neighbors interactively at dawn: support for the social dynamics hypothesis. Behav. Ecol. 19, 1192-1199.

Foote, J.R., Fitzsimmons, L.P., Mennill, D.J., Ratcliffe, L.M., 2008b. Tied to the nest: male black-capped chickadees decrease dawn chorus movement behaviour when their mate is fertile. Anim. Behav. 76, 1227-1233.

Forstmeier, W., Balsby, T.J.S., 2002. Why mated dusky warblers sing so much: Territory guarding and male quality announcement. Behaviour 139, 89-111.

Forstmeier, W., Nakagawa, S., Griffith, S.C., Kempenaers, B., 2014. Female extra-pair mating: adaptation or genetic constraint? Trends Ecol. Evol. 29, 456-464.

Freeman, S., 1987. Male red-winged blackbirds (Agelaius phoeniceus) assess the RHP of neighbors by watching contests. Behav. Ecol. Sociobiol. 21, 307-311.

Garamszegi, L.Z., 2004. Extrapair paternity and the evolution of bird song. Behav. Ecol. 15, 
$508-519$.

Garcia-Fernandez, V., Amy, M., Lacroix, A., Malacarne, G., Leboucher, G., 2010.

Eavesdropping on male singing interactions leads to differential allocation in eggs.

Ethology 116, 662-670.

Gerhardt, F., 2005. Food pilfering in larder-hoarding red squirrels (Tamiasciurus hudsonicus).

J. Mammal. 86, 108-114.

Gil, D., Gahr, M.C., 2002. The honesty of bird song: multiple constraints for multiple traits.

Trends Ecol. Evol. 17, 133-141.

Gil, D., Graves, J., Hazon, N., Wells, A., 1999a. Male attractiveness and differential testosterone investment in zebra finch eggs. Science. 286, 126-128.

Gil, D., Graves, J.A., Slater, P.J.B., 1999b. Seasonal patterns of singing in the willow warbler: evidence against the fertility announcement hypothesis. Anim. Behav. 58, 995-1000.

Gil, D., Leboucher, G., Lacroix, A., Cue, R., Kreutzer, M., 2004. Female canaries produce eggs with greater amounts of testosterone when exposed to preferred male song. Horm. Behav. 45, 64-70.

Gil, D., Llusia, D., 2020. The bird dawn chorus revisited., in: Aubin, T., Mathevon, N. (Eds.), Coding Strategies in Vertebrate Acoustic Communication. Springer Verlag, Heidelberg.

Gill, L.F., D’Amelio, P.B., Adreani, N.M., Sagunsky, H., Gahr, M.C., Maat, A., 2016. A minimum-impact, flexible tool to study vocal communication of small animals with precise individual-level resolution. Methods Ecol. Evol. 7, 1349-1358.

Godfrey, S.S., Bradley, J.K., Sih, A., Bull, C.M., 2012. Lovers and fighters in sleepy lizard land: where do aggressive males fit in a social network? Anim. Behav. 83, 209-215.

Goodale, E., Beauchamp, G., Magrath, R.D., Nieh, J.C., Ruxton, G.D., 2010. Interspecific information transfer influences animal community structure. Trends Ecol. Evol. 25, 354361.

Grabowska-Zhang, A.M., Sheldon, B.C., Hinde, C.A., 2012. Long-term familiarity promotes joining in neighbour nest defence. Biol. Lett. 8, 544-546.

Grabowska-Zhang, A.M., Wilkin, T.A., Sheldon, B.C., 2011. Effects of neighbor familiarity on reproductive success in the great tit (Parus major).

Grafen, A., 1990. Biological signals as handicaps. J. Theor. Biol. 144, 517-546.

Greives, T.J., Kingma, S.A., Kranstauber, B., Mortega, K., Wikelski, M., Oers, K. Van, Mateman, A.C., Ferguson, G.A., Hau, M., 2015. Costs of sleeping in: circadian rhythms influence cuckoldry risk in a songbird. Funct. Ecol. 29, 1300-1307.

Guilford, T., Dawkins, M.S., 1995. What are conventional signals? Anim. Behav. 49, 1689- 
1695.

Halfwerk, W., Bot, S., Buikx, J., van der Velde, M., Komdeur, J., ten Cate, C., Slabbekoorn, H., 2011. Low-frequency songs lose their potency in noisy urban conditions. Proc. Natl. Acad. Sci. U. S. A. 108, 14549-54.

Hall, M.L., Illes, A., Vehrencamp, S.L., 2006. Overlapping signals in banded wrens: longterm effects of prior experience on males and females. Behav. Ecol. 17, 260-269.

Hartig, F., 2019. DHARMa: residual diagnostics for hierarchical (multi-level/ mixed) regression models. $\mathrm{R}$ package version 0.2.6.

Hasselquist, D., Bensch, S., von Schantz, T., 1996. Correlation between male song repetoire, extra-pair paternity and offspring survival in the great reed warbler. Nature 381, 229232.

Hebets, E.A., Papaj, D.R., 2005. Complex signal function: developing a framework of testable hypotheses. Behav. Ecol. Sociobiol. 57, 197-214.

Helfer, B., Osiejuk, T.S., 2015. It takes all kinds in acoustic communication: a new perspective. Ethology 121, 315-326.

Henwood, K., Fabrick, A., 1979. A quantitative analysis of the dawn chorus: temporal selection for communicatory optimization. Am. Nat. 114, 260-274.

Hill, G.E., Farmer, K.L., 2005. Carotenoid-based plumage coloration predicts resistance to a novel parasite in the house finch. Naturwissenschaften 92, 30-34.

Hsu, Y.H., Schroeder, J., Winney, I., Burke, T., Nakagawa, S., 2015. Are extra-pair males different from cuckolded males? A case study and a meta-analytic examination. Mol. Ecol. 24, 1558-1571.

Hultsch, H., Todt, D., 1982. Temporal performance roles during vocal interactions in nightingales (Luscinia megarhynchos B.). Behav. Ecol. Sociobiol. 11, 253-260.

Humbird, S.K., Neudorf, D.L.H., 2008. The effects of food supplementation on extraterritorial behavior in female northern cardinals. Condor 110, 392-395.

Hyman, J., Hughes, M., 2006. Territory owners discriminate between aggressive and nonaggressive neighbours. Anim. Behav. 72, 209-215.

Järvi, T., Bakken, M., 1984. The function of the variation in the breast stripe of the great tit (Parus major). Anim. Behav. 590-596.

Jennions, M.D., Petrie, M., 2000. Why do females mate multiply? A review of the genetic benefits. Biol. Rev. 75, 21-64.

Johnson, J., Akerman, A., 1998. Watch and learn: preview of the fighting ability of opponents alters contest behaviour in rainbow trout. Anim. Behav. 56, 771-776. 
Kacelnik, A., 1979. The foraging efficiency of great tits (Parus major) in relation to light intensity. Anim. Behav. 27, 237-241.

Kacelnik, A., Krebs, J.R., 1983. The dawn chorus in the great tit (Parus major) - proximate and ultimate causes. Behaviour 83, 287-309.

Kalinowski, S.T., Taper, M.L., Marshall, T.C., 2007. Revising how the computer program cervus accommodates genotyping error increases success in paternity assignment. Mol. Ecol. 16, 1099-1106.

Kempenaers, B., Borgström, P., Loës, P., Schlicht, E., Valcu, M., 2010. Artificial night lighting affects dawn song, extra-pair siring success, and lay date in songbirds. Curr. Biol. 20, 1735-1739.

Kempenaers, B., Dhondt, A., 1993. Why do females engage in extra-pair copulations? A review of hypotheses and their predictions. Belgian J. Zool. 123, 93-103.

Kempenaers, B., Verheyen, G.R., Dhondt, A.A., 1997. Extrapair paternity in the blue tit (Parus caeruleus): female choice, male characteristics, and offspring quality. Behav. Ecol. Eeol 8, 481-492.

Kempenaers, B., Verheyen, G.R., van den Broeck, M., Burke, T., van Broeckhoven, C., Dhondt, A.A., 1992. Extra-pair paternity results from female preference for high-quality males in the blue tit. Nature 357, 494-496.

King, S.L., 2015. You talkin' to me ? Interactive playback is a powerful yet underused tool in animal communication research. Biol. Lett. 11, 20150403.

King, S.L., Harley, H.E., Janik, V.M., 2014. The role of signature whistle matching in bottlenose dolphins, Tursiops truncatus. Anim. Behav. 96, 79-86.

Kleven, O., Marthinsen, G., Lifjeld, J.T., 2006. Male extraterritorial forays, age and paternity in the socially monogamous reed bunting (Emberiza schoeniclus). J. Ornithol. 147, 468473.

Krebs, J., Ashcroft, R., Webber, M., 1978. Song repertoires and territory defence in the great tit. Nature 271, 539-542.

Krebs, J.R., Ashcroft, R., Orsdol, K. Van, 1981. Song matching in the great tit Parus major L. Anim. Behav. 29, 918-923.

Krist, M., Nádvorník, P., Uvírová, L., Bureš, S., 2005. Paternity covaries with laying and hatching order in the collared flycatcher Ficedula albicollis. Behav. Ecol. Sociobiol. 59, $6-11$.

Krokene, C., Anthonisen, K., Lifjeld, J.T., Amundsen, T., 1996. Paternity and paternity assurance behaviour in the bluethroat, Luscinia s. svecica. Anim. Behav. 52, 405-417. 
Krokene, C., Rigstad, K., Dale, M., Lifjeld, J.T., 1998. The function of extrapair paternity in blue tits and great tits: good genes or fertility insurance? Behav. Ecol. 9, 649-656.

Kunc, H.P., Amrhein, V., Naguib, M., 2006. Vocal interactions in nightingales, Luscinia megarhynchos: more aggressive males have higher pairing success. Anim. Behav. 72, $25-30$.

Kunc, H.P., Amrhein, V., Naguib, M., 2005. Seasonal variation in dawn song characteristics in the common nightingale. Anim. Behav. 70, 1265-1271.

Kurvers, R., van Oers, K., Nolet, B., Jonker, R.M., van Wieren, S.E., Prins, H.T., Ydenberg, R.C., 2010. Personality predicts the use of social information. Ecol. Lett. 829-837.

Kurvers, R.H.J.M., Eijkelenkamp, B., Van Oers, K., Van Lith, B., Van Wieren, S.E., Ydenberg, R.C., Prins, H.H.T., 2009. Personality differences explain leadership in barnacle geese. Anim. Behav. 78, 447-453.

Lambrechts, M., Dhondt, A., 1986. Male quality, reproduction, and survival in the great tit (Parus major). Behav. Ecol. Sociobiol. 19, 57-63.

Langemann, U., Tavares, J.P., Peake, T.M., McGregor, P.K., Mc Gregor, P.K., 2000. Response of great tits to escalating patterns of playback. Behaviour 137, 451-471. Larmuseau, M.H.D., van den Berg, P., Claerhout, S., Calafell, F., Boattini, A., Gruyters, L., Vandenbosch, M., Nivelle, K., Decorte, R., Wenseleers, T., 2019. A historical-genetic reconstruction of human extra-pair paternity. Curr. Biol. 29, 1-6.

Larsen, K.W., Boutin, S., 1994. Movements, survival, and settlement of red squirrel (Tamiasciurus hudsonicus) offspring. Ecology 75, 214-223.

Leboucher, G., Pallot, K., 2004. Is he all he says he is? Intersexual eavesdropping in the domestic canary, Serinus canaria. Anim. Behav. 68, 957-963.

Lein, R.M., 2007. Patterns of dawn singing by buff-breasted flycatchers. J. F. Ornithol. 78, 343-351.

Leitão, A., Riebel, K., 2003. Are good ornaments bad armaments? Male chaffinch perception of songs with varying flourish length. Anim. Behav. 66, 161-167.

Lemel, J., Wallin, K., 1993. Status signalling, motivational condition and dominance: an experimental study in the reat tit, Parus major. Anim. Behav. 45, 549-558.

Limbourg, T., Mateman, A.C., Andersson, S., Lessells, C. (Kate) M., 2004. Female blue tits adjust parental effort to manipulated male UV attractiveness. Proc. R. Soc. B Biol. Sci. 271, 1903-1908.

Liu, W.-C., 2004. The effect of neighbours and females on dawn and daytime singing behaviours by male chipping sparrows. Anim. Behav. 68, 39-44. 
Liu, W.-C., Kroodsma, D.E., 2007. Dawn and daytime singing behavior of chipping sparrows (Spizella passerina). Auk 124, 44-52.

Logue, D.M., Forstmeier, W., 2008. Constrained performance in a communication network: implications for the function of song-type matching. Am. Nat. 172, 34-41.

Lubjuhn, T., Strohbach, S., Brun, J., Gerken, T., Epplen, J.T., 1999. Extra-pair paternity in great tits (Parus major) - A long term study. Behaviour 136, 1157-1172.

Mace, R., 1987. The dawn chorus in the great tit Parus major is directly related to female fertility. Nature 330, 745-746.

Mace, R., 1986. Importance of female behaviour in the dawn chorus. Anim. Behav. 34, 621622.

Marshall, T.C., Slate, J., Kruuk, L.E.B., Pemberton, J.M., 1998. Statistical confidence for likelihood-based paternity inference in natural populations. Mol. Ecol. 639-655.

Masco, C., Allesina, S., Mennill, D.J., Pruett-Jones, S., 2015. The Song Overlap Null model Generator (SONG): a new tool for distinguishing between random and non-random song overlap. Bioacoustics 4622, 1-12.

Mayer, M., Zedrosser, A., Rosell, F., 2017. Extra-territorial movements differ between territory holders and subordinates in a large, monogamous rodent. Sci. Rep. 7, 15261.

McGregor, P.K., 1993. Signalling in territorial systems: a context for individual identification, ranging and eavesdropping. Philos. Trans. R. Soc. London B Biol. Sci. 340, 237-244.

McGregor, P.K., 1991. The singer and the song: On the receiving end of bird song. Biol. Rev. Camb. Philos. Soc. 66, 57-81.

McGregor, P.K., Dabelsteen, T., Shepherd, M., Pedersen, S.B., 1992. The signal value of matched singing in great tits: evidence from interactive playback experiments. Anim. Behav. 43, 987-998.

McGregor, P.K., Krebs, J.R., Perrins, C.M., 1981. Song repertoires and lifetime reproductive success in the great tit (Parus major). Am. Nat. 118, 149-159.

McGregor, P.K., Otter, K.A., Peake, T.M., 2000. Communication networks: receiver and signaller perspectives., in: Epsmark, Y., Amundsen, T., Rosenqvist, G. (Eds.), Animal Signals. Adaptive Significance of Signalling and Signal Design in Animal Communication. Tapir, Trondheim, Norway, pp. 405-416.

McGregor, P.K., Peake, T.M., 2000. Communication networks: social environments for receiving and signalling behaviour. Acta Ethol. 2, 71-81.

McNamara, J.M., Mace, R.H., Houston, A.I., 1987. Optimal daily routines of singing and foraging in a bird singing to attract a mate. Behav. Ecol. Sociobiol. 20, 399-405. 
Mennill, D.J., Battiston, M., Wilson, D.R., Foote, J.R., Doucet, S.M., 2012a. Field test of an affordable, portable, wireless microphone array for spatial monitoring of animal ecology and behaviour. Methods Ecol. Evol. 3, 704-712.

Mennill, D.J., Boag, P.T., Ratcliffe, L.M., 2003. The reproductive choices of eavesdropping female black-capped chickadees, Poecile atricapillus. Naturwissenschaften 90, 577-582.

Mennill, D.J., Burt, J.M., Fristrup, K.M., Vehrencamp, S.L., 2006. Accuracy of an acoustic location system for monotoring the position of duetting songbirds in tropical forest. J. Acoust. Soc. Am. 119, 2832-2839.

Mennill, D.J., Doucet, S.M., Newman, A.E.M., Williams, H., Moran, I.G., Thomas, I.P., Woodworth, B.K., Bornais, M.M.K., Norris, D.R., 2019. Eavesdropping on adult vocal interactions does not enhance juvenile song learning: an experiment with wild songbirds. Anim. Behav. 155, 67-75.

Mennill, D.J., Doucet, S.M., Ward, K.-A.A., Maynard, D.F., Otis, B., Burt, J.M., 2012b. A novel digital telemetry system for tracking wild animals: a field test for studying mate choice in a lekking tropical bird. Methods Ecol. Evol. 3, 663-672.

Mennill, D.J., Ramsay, S.M., Boag, P.T., Ratcliffe, L.M., 2004. Patterns of extrapair mating in relation to male dominance status and female nest placement in black-capped chickadees. Behav. Ecol. 15, 757-765.

Mennill, D.J., Ratcliffe, L.M., 2004. Do male black-capped chickadees eavesdrop on song contests? A multi-speaker playback experiment. Behaviour 141, 125-139.

Mennill, D.J., Ratcliffe, L.M., Boag, P.T., 2002. Female eavesdropping on male song contests in songbirds. Science. 296, 873.

Messier, F., 1985. Solitary living and extraterritorial movements of wolves in relation to social status and prey abundance. Can. J. Zool. 63, 239-245.

Metzgar, L.H., 1967. An experimental comparison of screech owl predation on resident and transient white-footed mice (Peromyscus leucopus). J. Mammal. 48, 387.

Møller, A.P., 1990. Changes in the size of avian breeding territories in relation to the nesting cycle. Anim. Behav. 40, 1070-1079.

Møller, A.P., 1988. Spatial and temporal distribution of song in the yellowhammer Emberiza citrinella. Ethology 78, 321-331.

Moran, I.G., Lukianchuk, K.C., Doucet, S.M., Newman, A.E.M., Williams, H., Norris, D.R., Mennill, D.J., 2019. Diel and seasonal patterns of variation in the singing behaviour of savannah sparrows (Passerculus sandwichensis). Avian Res. 10, 1-8.

Morinay, J., Forsman, J.T., Germain, M., Doligez, B., 2020. Behavioural traits modulate the 
use of heterospecific social information for nest site selection: experimental evidence from a wild bird population. Proc. R. Soc. B Biol. Sci. 287, 20200265.

Morton, E.S., 1996. A comparison of vocal behavior among tropical and tem- perate passerine birds, in: Kroodsma, D.E., Miller, E.H. (Eds.), Ecology and Evolution of Acoustic Communication in Birds. Cornell University Press, Ithaca, NY, pp. 258-268.

Mountjoy, D.J., Lemon, R.E., 1991. Song as an attractant for male and female european starlings, and the influence of song complexity on their response. Behav. Ecol. Sociobiol. 28, 97-100.

Mutzel, A., Dingemanse, N.J., Araya-Ajoy, Y.G., Kempenaers, B., 2013. Parental provisioning behaviour plays a key role in linking personality with reproductive success. Proc. R. Soc. B Biol. Sci. 280.

Naguib, M., 2005. Singing interactions in songbirds: implications for social relations and territorial settlement., in: McGregor, P.K. (Ed.), Animal Communication Networks. Cambridge University Press, Cambridge, pp. 300-319.

Naguib, M., Altenkamp, R., Griessmann, B., 2001. Nightingales in space: song and extraterritorial forays of radio tagged song birds. J. Ornithol. 142, 306-312.

Naguib, M., Amrhein, V., Kunc, H.P., 2004. Effects of territorial intrusions on eavesdropping neighbors: communication networks in nightingales. Behav. Ecol. 15, 1011-1015.

Naguib, M., Diehl, J., van Oers, K., Snijders, L., 2019. Repeatability of signalling traits in the avian dawn chorus. Front. Zool. 16:27.

Naguib, M., Fichtel, C., Todt, D., 1999. Nightingales respond more strongly to vocal leaders of simulated dyadic interactions. Proc. R. Soc. London. Ser. B Biol. Sci. 266, 537-542.

Naguib, M., Kipper, S., 2006. Effects of different levels of song overlapping on singing behaviour in male territorial nightingales (Luscinia megarhynchos). Behav. Ecol. Sociobiol. 59, 419-426.

Naguib, M., Kunc, H.P., Sprau, P., Roth, T., Amrhein, V., 2011. Communication networks and spatial ecology in nightingales. Adv. Study Behav. 43, 239-271.

Naguib, M., Mennill, D.J., 2010. The signal value of birdsong: empirical evidence suggests song overlapping is a signal. Anim. Behav. 80, 11-12.

Naguib, M., Riebel, K., 2006. Bird song: a key model in animal communication, in: Brown, K. (Ed.), Encyclopedia for Language and Linguistics. Elsevier, pp. 40-53.

Naguib, M., Todt, D., 1997. Effects of dyadic vocal interactions on other conspecific receivers in nightingales. Anim. Behav. 54, 1535-1544.

Naguib, M., Van Rooij, E.P., Snijders, L., Van Oers, K., 2016. To sing or not to sing: 
seasonal changes in singing vary with personality in wild great tits. Behav. Ecol. 27, 932-938.

Naguib, M., Wiley, R.H., 2001. Estimating the distance to a source of sound: mechanisms and adaptations for long-range communication. Anim. Behav. 62, 825-837.

Neudorf, D.L., Stutchbury, B.J.M., Piper, W.H., 1997. Covert extraterritorial behavior of female hooded warblers. Behav. Ecol. 8, 595-600.

Nilsson, A.L.K., Nilsson, J.Å., Alerstam, T., Bäckman, J., 2010. Migratory and resident blue tits Cyanistes caeruleus differ in their reaction to a novel object. Naturwissenschaften 97, 981-985.

Norris, K., 1993. Heritable variation in a plumage indicator of viability in male great tits Parus major. Nature 362, 537-539.

Norris, K.J., 1990. Female choice and the evolution of the conspicuous plumage coloration of monogamous male great tits. Behav. Ecol. Sociobiol. 26, 129-138.

Nowicki, S., Searcy, W.A., Hughes, M.E., 1998. The territory defense function of song in song sparrows: a test with the speaker occupation design. Behavior 135, 615-628.

Odom, K.J., Hall, M.L., Riebel, K., Omland, K.E., Langmore, N.E., 2014. Female song is widespread and ancestral in songbirds. Nat. Commun. 5, 1-6.

Oliveira, R.F., McGregor, P.K., Latruffe, C., 1998. Know thine enemy: fighting fish gather information from observing conspecific interactions. Proc. R. Soc. London B Biol. Sci. 265, 1045-1049.

Ophir, A.G., Galef, B.G., 2003. Female Japanese quail that 'eavesdrop' on fighting males prefer losers to winners. Anim. Behav. 66, 399-407.

Otter, K., Chruszcz, B., Ratcliffe, L.M., 1997. Honest advertisement and song output during the dawn chorus of black-capped chickadees. Behav. Ecol. 8, 167-173.

Otter, K., McGregor, P.K., Terry, A.M.R., Burford, F.R.L., Peake, T.M., Dabelsteen, T., 1999. Do female great tits (Parus major) assess males by eavesdropping? A field study using interactive song playback. Proc. R. Soc. B Biol. Sci. 266, 1305-1309.

Otter, K., Ratcliffe, L.M., 2005. Enlightened decisions: female assessment and communication networks., in: McGregor, P.K. (Ed.), Animal Communication Networks. Cambridge University Press, Cambridge, pp. 133-151.

Otter, K., Stewart, I.R.K., McGregor, P.K., Terry, A.M.R., Burke, T., Ian, R., 2001. Extrapair paternity among great tits Parus major following manipulation of male signals. J. Avian Biol. 32, 338-344.

Pärt, T., 1991. Is dawn singing related to paternity insurance? The case of the collared 
flycatcher. Anim. Behav. 41, 451-456.

Patrick, S.C., Chapman, J.R., Dugdale, H.L., Quinn, J.L., Sheldon, B.C., 2012. Promiscuity, paternity and personality in the great tit. Proc. R. Soc. B-Biological Sci. 279, 1724-1730.

Peake, T.M., Terry, A.M.R., McGregor, P.K., Dabelsteen, T., 2001. Male great tits eavesdrop on simulated male-to-male vocal interactions. Proc. R. Soc. B Biol. Sci. 268, 1183-1187.

Peake, T.M., Terry, A.M.R., McGregor, P.K., Dabelsteen, T., Terry, M.R., McGregor, P.K., Dabelsteen, T., 2002. Do great tits assess rivals by combining direct experience with information gathered by eavesdropping? Proc. R. Soc. London. Ser. B Biol. Sci. 269, 1925-1929.

Pedersen, M.C., Dunn, P.O., Whittingham, L.A., 2006. Extraterritorial forays are related to a male ornamental trait in the common yellowthroat. Anim. Behav. 72, 479-486.

Picq, S., Scotti, M., Puebla, O., 2019. Behavioural syndromes as a link between ecology and mate choice: a field study in a reef fish population. Anim. Behav. 150, 219-237.

Pinxten, R., Eens, M., 1998. Male starlings sing most in the late morning, following egglaying: a strategy to protect their paternity? Behaviour 135, 1197-1211.

Pitcher, T.E., Stutchbury, B.J.M., 2000. Extraterritorial forays and male parental care in hooded warblers. Anim. Behav. 59, 1261-1269.

Poesel, A., Dabelsteen, T., 2005. Territorial responses of male blue tits to simulated dynamic intrusions: effects of song overlap and intruder location. Anim. Behav. 70, 1419-1427.

Poesel, A., Kunc, H.P., Foerster, K., Johnsen, A., Kempenaers, B., 2006. Early birds are sexy: male age, dawn song and extrapair paternity in blue tits, Cyanistes (formerly Parus) caeruleus. Anim. Behav. 72, 531-538.

Price, J.J., 2015. Rethinking our assumptions about the evolution of bird song and other sexually dimorphic signals. Front. Ecol. Evol. 3, 40.

Quesada, J., Senar, J.C., 2006. Comparing plumage colour measurements obtained directly from live birds and from collected feathers: the case of the great tit Parus major. J. Avian Biol. 37, 609-616.

Qvarnström, A., Forsgren, E., 1998. Should females prefer dominant males? Trends Ecol. Evol. 13, 498-501.

R Core Team, 2019. R: A language and environment for statistical computing.

Riebel, K., Hall, M.L., Langmore, N.E., 2005. Female songbirds still struggling to be heard. Trends Ecol. Evol. 20, 419-20.

Riebel, K., Odom, K.J., Langmore, N.E., Hall, M.L., 2019. New insights from female bird song: towards an integrated approach to studying male and female communication roles. 
Biol. Lett. 15, 20190059.

Ritschard, H., Brumm, M., 2012. Zebra finch song reflects current food availability. Evol. Ecol. 26, 801-812.

Roth, T., Sprau, P., Schmidt, R., Naguib, M., Amrhein, V., 2009. Sex-specific timing of mate searching and territory prospecting in the nightingale: nocturnal life of females. Proc. R. Soc. B-Biological Sci. 276, 2045-2050.

Saladin, V., Bonfils, D., Binz, T., Richner, H., 2003. Isolation and characterization of 16 microsatellite loci in the great tit Parus major. Mol. Ecol. Notes 3, 520-522.

Schlicht, L., Girg, A., Loës, P., Valcu, M., Kempenaers, B., 2012. Male extrapair nestlings fledge first. Anim. Behav. 83, 1335-1343.

Schlicht, L., Valcu, M., Kempenaers, B., 2015. Male extraterritorial behavior predicts extrapair paternity pattern in blue tits, Cyanistes caeruleus. Behav. Ecol. 26, 1404-1413.

Schlicht, L., Valcu, M., Loës, P., Girg, A., Kempenaers, B., 2014. No relationship between female emergence time from the roosting place and extrapair paternity. Behav. Ecol. 25, $650-659$.

Schmidt, K.A., Whelan, C.J., 2005. Quantifying male wood thrush nest-attendance and its relationship to nest success. Condor 107, 138-144.

Schmidt, R., Amrhein, V., Kunc, H.P., Naguib, M., 2007. The day after: effects of vocal interactions on territory defence in nightingales. J. Anim. Ecol. 76, 168-173.

Schuett, W., Dall, S.R.X., Royle, N.J., 2011. Pairs of zebra finches with similar "personalities" make better parents. Anim. Behav. 81, 609-618.

Searcy, W.A., Anderson, R.C., Nowicki, S., 2006. Bird song as a signal of aggressive intent. Behav. Ecol. Sociobiol. 60, 234-241.

Searcy, W.A., Beecher, M.D., 2011. Continued scepticism that song overlapping is a signal. Anim. Behav. 81.

Searcy, W.A., Beecher, M.D., 2009. Song as an aggressive signal in songbirds. Anim. Behav. 78, 1281-1292.

Searcy, W.A., Yasukawa, K., 1996. Song and female choice, in: Kroodsma, D.E., Miller, E.H. (Eds.), Ecology and Evolution of Acoustic Communication in Birds. Cornell University Press, Ithaca, pp. 454-473.

Senar, J.C., Negro, J.J., Quesada, J., Ruiz, I., Garrido, J., 2008. Two pieces of information in a single trait? The yellow breast of the great tit (Parus major) reflects both pigment acquisition and body condition. Behaviour 145, 1195-1210.

Sexton, K., Murphy, M.T., Redmond, L.J., Dolan, A.C., 2007. Dawn song of eastern 
kingbirds: intrapopulation variability and sociobiological correlates. Behaviour 144, $1273-1295$.

Sheldon, B.C., 1994. Sperm competition in the chaffinch: the role of the female. Anim. Behav. 47, 163-173.

Sih, A., Bell, A., Johnson, J.C., 2004. Behavioral syndromes: an ecological and evolutionary overview. Trends Ecol. Evol. 19, 372-378.

Silk, J.B., 1999. Male bonnet macaques use information about third-party rank relationships to recruit allies. Anim. Behav. 58, 45-51.

Slagsvold, T., 1996. Dawn and dusk singing of male american robins in relation to female behavior. Wilson Bull. 108, 507-515.

Smit, J.A.H., van Oers, K., 2019. Personality types vary in their personal and social information use. Anim. Behav. 151, 185-193.

Smith, S.M., 1988. Extra-pair copulations in black-capped chickadees: the role of the female. Behavior 107, 15-23.

Snijders, L., Naguib, M., 2017. Communication in animal social networks: a missing link? Adv. Study Behav. 49, 297-359.

Snijders, L., van der Eijk, J., van Rooij, E.P., de Goede, P., van Oers, K., Naguib, M., 2015. Song trait similarity in great tits varies with social structure. PLoS One 10.

Snijders, L., van Oers, K., Naguib, M., 2017a. Sex-specific responses to territorial intrusions in a communication network: evidence from radio-tagged great tits. Ecol. Evol. 7, 918927.

Snijders, L., van Rooij, E.P., Burt, J.M., Hinde, C.A., van Oers, K., Naguib, M., 2014. Social networking in territorial great tits: slow explorers have the least central social network positions. Anim. Behav. 98, 95-102.

Snijders, L., Weme, L.E.N., de Goede, P., Savage, J.L., van Oers, K., Naguib, M., $2017 b$. Context-dependent effects of radio transmitter attachment on a small passerine. J. Avian Biol. 48, 650-659.

Soma, M., Garamszegi, L.Z., 2011. Rethinking birdsong evolution: meta-analysis of the relationship between song complexity and reproductive success. Behav. Ecol. 22, 363371.

Spiegel, O., Leu, S.T., Bull, C.M., Sih, A., 2017. What's your move? Movement as a link between personality and spatial dynamics in animal populations. Ecol. Lett. 20, 3-18.

Sprau, P., Roth, T., Amrhein, V., Naguib, M., 2014. Effects of previous intrusion pressure on territorial responses in nightingales. J. Ornithol. 155, 111-119. 
Staicer, C.A., Spector, D.A., Horn, A.G., 1996. The dawn chorus and other diel patterns in acoustic signaling., in: Kroodsma, D.E., Miller, E.H. (Eds.), Ecology and Evolution of Acoustic Communication in Birds. Cornell University Press, Ithaca, N.Y, pp. 426-453.

Stoddard, M.C., Prum, R.O., 2008. Evolution of avian plumage color in a tetrahedral colour space: a phylogenetic analysis of new world buntings. Am. Nat. 171, 755-776.

Stoddard, P.K., 1996. Vocal recognition of territorial passerines., in: Kroodsma, D.E., Miller, E.H. (Eds.), Ecology and Evolution of Acoustic Communication in Birds. Cornell University Press, Ithaca, New York, pp. 356-374.

Strohbach, S., Curio, E., Bathen, A., Epplen, J.T., Lubjuhn, T., 1998. Extrapair paternity in the great tit (Parus major): a test of the "good genes" hypothesis. Behav. Ecol. 9, 388396.

Stutchbury, B.J.M., 1998. Extra-pair mating effort of male hooded warblers, Wilsonia citrina. Anim. Behav. 55, 553-561.

Stutchbury, B.J.M., Pitcher, T.E., Norris, D.R., Tuttle, E.M., Gonser, R.A., 2005. Does male extra-territory foray effort affect fertilization success in hooded warblers Wilsonia citrina ? J. Avian Biol. 36, 471-477.

Tanvez, A., Béguin, N., Chastel, O., Lacroix, A., Leboucher, G., 2004. Sexually attractive phrases increase yolk androgens deposition in canaries (Serinus canaria). Gen. Comp. Endocrinol. 138, 113-20.

Temeles, E.J., 1994. The role of neighbours in territorial systems: when are they "dear enemies"? Anim. Behav. 47, 339-350.

Templeton, C.N., Akcay, C., Campbell, S.E., Beecher, M.D., Akçay, C., Campbell, S.E., Beecher, M.D., 2010. Juvenile sparrows preferentially eavesdrop on adult song interactions. Proc. R. Soc. B Biol. Sci. 277, 447-453.

Todt, D., 1981. On functions of vocal matching: effect of counter-replies on song post choice and singing. Z. Tierpsychol. 57, 73-93.

Todt, D., Naguib, M., 2000. Vocal interactions in birds: the use of song as a model in communication. Adv. Study Behav. Vol. 29 29, 247-296.

Toth, C.A., Mennill, D.J., Ratcliffe, L.M., 2012. Evidence for multicontest eavesdropping in chickadees. Behav. Ecol. 23, 836-842.

Trillo, P.A., Vehrencamp, S.L., 2005. Song types and their structural features are associated with specific contexts in the banded wren. Anim. Behav. 70, 921-935.

Uller, T., Olsson, M., 2008. Multiple paternity in reptiles: patterns and processes. Mol. Ecol. $17,2566-2580$. 
Valone, T.J., Templeton, J.J., 2002. Public information for the assessment of quality: a widespread social phenomenon. Philos. Trans. R. Soc. London. Ser. B Biol. Sci. 357, $1549-1557$.

van Balen, J.H., 1973. A comparative study of the breeding ecology of the great tit Parus major in different habitats. Ardea 55, 1-93.

Van Balen, J.H., 1967. The significance of variations in body weight and wing length in the great tit, Parus major. Ardea 55, 1-59.

van Oers, K., Drent, P.J., De Goede, P., Van Noordwijk, A.J., 2004. Realized heritability and repeatability of risk-taking behaviour in relation to avian personalities. Proc. R. Soc. B Biol. Sci. 271, 65-73.

van Oers, K., Drent, P.J., Dingemanse, N.J., Kempenaers, B., 2008. Personality is associated with extrapair paternity in great tits, Parus major. Anim. Behav. 76, 555-563.

van Oers, K., Klunder, M., Drent, P.J., 2005. Context dependence of personalities: risk-taking behavior in a social and a nonsocial situation. Behav. Ecol. 16, 716-723.

van Oers, K., Naguib, M., 2013. Avian Personality, in: Carere, C., Maestripieri, D. (Eds.), Animal Personalities: Behavior, Physiology and Evolution. The University of Chicago Press, Chicago IL and London UK, pp. 66-95.

van Overveld, T., Matthysen, E., 2013. Personality and information gathering in free-ranging great tits. PLoS One 8, e54199.

van Overveld, T., Matthysen, E., 2010. Personality predicts spatial responses to food manipulations in free-ranging great tits (Parus major). Biol. Lett. 6, 187-190.

Vehrencamp, S.L., 2001. Is song-type matching a conventional signal of aggressive intentions? Proc. R. Soc. B Biol. Sci. 268, 1637-1642.

Venables, W.N., Ripley, B.D., 2002. Modern Applied Statistics with S, 4th ed. Springer, New York.

Ward, M.P., 2005. Habitat selection by dispersing yellow-headed blackbirds: evidence of prospecting and the use of public information. Oecologia 145, 650-657.

Ward, M.P., Alessi, M., Benson, T.J., Chiavacci, S.J., 2014. The active nightlife of diurnal birds: extraterritorial forays and nocturnal activity patterns. Anim. Behav. 88, 175-184.

Westneat, D.F., 1993. Temporal patterns of within-pair copulations, male mate-guarding, and extra-pair events in eastern red-winged blackbirds (Agelaius phoeniceus). Behaviour 124, 267-290.

Westneat, D.F., Stewart, I.R., 2003. Extra-pair paternity in birds: causes, correlates, and conflict. Annu. Rev. Ecol. Evol. Syst. 34, 365-396. 
While, G.M., Sinn, D.L., Wapstra, E., 2009. Female aggression predicts mode of paternity acquisition in a social lizard. Proc. R. Soc. B Biol. Sci. 276, 2021-2029.

Wilson, A.D.M., McLaughlin, R.L., 2007. Behavioural syndromes in brook charr, Salvelinus fontinalis: prey-search in the field corresponds with space use in novel laboratory situations. Anim. Behav. 74, 689-698.

Wilson, J.D., 1992. A re-assessment of the significance of status signalling in populations of wild great tits, Parus major. Anim. Behav. 43, 999-1009.

Wong, B.B.M., Candolin, U., 2005. How is female mate choice affected by male competition? Biol. Rev. 80, 559-571.

Yasukawa, K., 1981. Song and territory defense in the red-winged blackbird. Auk 98, 185187.

Young, A.J., Monfort, S.L., 2009. Stress and the costs of extra-territorial movement in a social carnivore. Biol. Lett. 5, 439-441.

Zahavi, A., 1975. Mate selection - selection for a handicap. J. Theor. Biol. 53, 205-214. 


\section{Summary}

Bird song has, alongside elaborate plumage displays, become a textbook example of a sexually selected signal (Andersson, 1994; Bradbury and Vehrencamp, 1998). As many other conspicuous male ornaments it serves both as an "ornament and armament" (Berglund et al., 1996): its general dual function in female attraction and male-male competition is supported by many correlative and experimental studies (Catchpole and Slater, 2008; McGregor, 1991; Naguib and Riebel, 2006; Searcy and Yasukawa, 1996). As song is a long-range signal it can be directed at and heard by multiple receivers simultaneously (McGregor et al., 2000; McGregor and Peake, 2000), making it inherently difficult to determine to which audience a bird precisely is singing at a given time. Thus, to understand the role of song in both mate attraction and male-male competition, it is crucial to obtain information of all participating individuals and their response, but receiver movements are often cryptic and difficult to follow (Bircher and Naguib, 2020; Snijders and Naguib, 2017). Thus, many aspects of how receivers use this signal are still not well understood. Moreover, bird song is highly interactive and dynamic and some of its information may be conveyed only through specific matching and timing strategies during male-male vocal interactions, not through solo song alone (Burt and Vehrencamp, 2005; Catchpole and Slater, 2008; Naguib, 2005; Todt and Naguib, 2000). What theses singing patterns signal exactly about interacting males and how eavesdropping receivers may use the information conveyed by them is still debated (Helfer and Osiejuk, 2015; Naguib and Mennill, 2010; Searcy and Beecher, 2011, 2009). In my thesis I combined automated radiotracking of both females and males throughout the breeding season with acoustic (array-) recordings, interactive playback experiments and microsatellite analysis to study the role of male song in the spatial behavior and reproductive decisions of the great tit (Parus major).

In Chapter 2, I examined the role of dawn song in female attraction and male-male competition. This well-known peak in bird singing activity just before sunrise is thought to play an important role in territory defense and attracting females for extra-pair copulations (Gil and Llusia, 2020; Kacelnik and Krebs, 1983; Staicer et al., 1996). However, we know little about the actual spatial behavior of potential receivers, both females and males, around this time compared to the rest of the day. Here I quantified movements of females and males to other territories ("forays") in relation to male dawn song, plumage ornaments, and extra-pair paternity. I show that the overall foraying behavior of both males and females during the breeding season is associated with dawn song characteristics, but not plumage ornaments, of territorial males. In contrast to what would 
be expected based on the proposed function of dawn song as a "keep out "- signal to other males and attraction signal to females, the results suggest that females do not visit areas more often when resident males sing more elaborately at dawn and males are more likely to visit territories of males that sing very actively at dawn. Moreover, neither female nor male forays were associated with the occurrence of extra-pair offspring. These findings suggest that foraying in great tits may not primarily be related to extra-pair mating, and both females and males may use the information contained in dawn song in different ways than previously thought, emphasizing that singing behavior, the information conveyed by it and how receivers make use of this information may change depending on the time window considered.

Foraying off-territory has often been proposed to serve the search of extra-pair copulations, but the results in Chapter 2 do not suggest the extra-pair mating is a primary reason for foraying off-territory in the great tit. Forays could have multiple other potential benefits, such as access to additional food sources or social and environmental information (Doligez et al., 2004a, 2002; Messier, 1985). However, forays can also be costly as they could lead for example to higher predation risk (Larsen and Boutin, 1994; Metzgar, 1967) or higher physiological stress levels (Young and Monfort, 2009). Consistent behavioral differences (personality) associated with the likelihood to take risks (Sih et al., 2004; van Oers and Naguib, 2013) may thus be an important factor that influences the off-territory movements of both females and males. In Chapter 5, I thus explored the relationship between exploratory behavior, an established proxy for a personality trait, and extra-territorial foraying behavior. Here I showed that individual personality was not associated with foraying activity. However, male foraying behavior is associated with both a male's own and his partner's personality with faster exploring males making more forays when paired with a slow exploring female, suggesting that males might trade-off costs and benefits of moving off-territory differently, depending on their own personality in combination with that of their partner.

Some of the information in male singing may be conveyed only through specific patterns in interactions between males, not by each signal alone (Catchpole and Slater, 2008; Dabelsteen, 1992; McGregor et al., 2000; Naguib, 2005) and eavesdropping on male-male song interactions is thus thought to be low-cost way of gathering information about relative differences between individuals that are important for the assessment of rivals or mates (Naguib, 2005; Todt and Naguib, 2000; Valone and Templeton, 2002). In Chapter 3 and Chapter 4 therefore examined the role of male-male singing interactions in male-male competition and female reproductive 
decisions. Using an interactive playback experiment, I tested in Chapter 3 whether female and male great tits use information gained by eavesdropping on male singing interactions to assess rivals and (potential) mates. Here I engaged territorial males in a song contests with either a more or less challenging simulated intruder and followed male and female movements before and after interactions by radio-tracking, determined paternity using microsatellite analysis and maternal investment by quantifying egg weights and provisioning behavior. In Chapter 4, I examined whether asymmetries in the use of specific singing strategies, song type matching and overlapping, in male-male song contests reflect relative differences between males in a number of characteristics that would be relevant for eavesdroppers in territory disputes or mate assessment. Using an array of programmable recorders I quantified levels of song type matching and overlapping during naturally occurring singing interactions between territorial males in early spring and compared these singing behaviors to winter dominance rank, breeding box quality, body condition, plumage ornamentation (size of black breast stripe), male provisioning behavior (feeding hours and rates) nestling success (fledging weight of genetic and foster chicks by males). However, my findings from both Chapter 3 and Chapter 4 provide no evidence that eavesdropping on male singing interactions provides females or males with information that is important in the assessment of males, either mates or rivals. In Chapter 3 males did not respond differently to a more challenging than to a less challenging intruder and their mates did not change their foraying behavior or the reproductive investment. Females with extra-pair offspring did thereby not differ in their spatial response from females without extrapair offspring. Moreover, neither female nor male neighbors changed their foraying behavior after playbacks. These findings do not suggest that females use information conveyed in song interactions of their mate with rivals in reproductive decisions or that information gathered by eavesdropping on interactions influences close-range associations between individuals in a territorial neighborhood. In Chapter 4 male singing behavior in interactions did not reflect relative differences between opponents in any of the individual characteristics measured. What information may be conveyed by certain singing patterns in male song contests and how different receivers might use this information is still somewhat debated (Helfer and Osiejuk, 2015; Naguib and Mennill, 2010; Searcy and Beecher, 2011, 2009). The use and importance of singing patterns such as type matching and overlapping may be highly time and context specific and my results emphasize the need for further research to understand whether, when and how males and females use any information gathered by eavesdropping on such interactions. 


\section{Acknowledgements}

It would not have been possible to write this thesis without the support of many others who have generously offered their advice and help during the last years. Above all, I would like to thank my supervisors for their guidance throughout the project: Marc, thank you for giving me this fantastic opportunity to start working on bird song and follow my research interests, both in the Netherlands and abroad. It was great to have a supervisor that was just as excited about the project and made time for discussions whenever possible. Kees, thank you for always having an open door and the many hours spent discussing ideas and analysis methods. Your input often made things so much clearer to me and your encouragement made a big difference when things went wrong. I am so grateful to have had you as an advisor during the start of my $\mathrm{PhD}$, Camilla - you often offered a different perspective and much inspiration, for my project and research questions, but also science in general.

I was lucky to be able to work at two institutions at the same time for my project and would like to thank all members of the Behavioral Ecology group at Wageningen University and the Animal Ecology department at NIOO-KNAW for creating such a great place to learn and work. A big thank you to everyone who joined the "big room" at BHE throughout my $\mathrm{PhD}$ years for the lively coffee breaks, science discussions and sharing ups and downs: Lysanne, Lies, James, Elske, Anne, Katharina, Shana, Filipe, Catarina, Nady, Nada, Haitham, Khaled, Mohamed, Miriam and Elizabeth. Jerine and Hugo, at some point the "big room" at BHE was rather small with just the three of us - thank you for being great $\mathrm{PhD}$ colleagues and especially for supporting me during the last stretch as "paranymphs". Lydia, thank you so much for all your support and hard work throughout my entire project. A big thank you to everyone who made my "NIOO days" so enjoyable: Piet, thank you for patiently introducing me to working with birds and your enthusiasm and good spirit in the field. Special thanks also to Christa and Martijn for all your help with the work in the lab and hunting down missing blood samples. And of course, thank you to all my NIOO field- and office colleagues: Lucia, Jip, Irene, Els, Barbara, Davide, Bernice, Krista, Eva, and Melanie. You always made me look forward to switch work places for a bit.

So many amazing helpers have contributed to the data collection and/or analysis during this project and without them this thesis would not have been possible. Most importantly, I would like to acknowledge all students that have joined during the years: Mara, Thomas, Maeliss, Laura, Hongye, Amelia, Femke, Lisanne, Sander, Bas, Rahman, Bernice G., Louis, Guido 
and Beril. Thank you for the many hours in the field and office. I am sure I have learnt as much from you as you have from me. Special thanks also to everyone that has joined the "tagging nights" and stayed late to help with catching birds for the deployment of many Encounternet tags and to Liam and Callum for sharing their R expertise.

Last, but certainly not least, I would like to thank my family for always being supportive and patiently listening to my stories of days spent listening to birds. I would never have reached this point without your support. 


\section{Curriculum vitae}

I have been fascinated by the complexity of human language ever since having to learn foreign languages and wondered about how and why it evolved. Combined with my passion for observing wildlife, this made me curious about communication in other species. After completing my BSc degree in Environmental Sciences at ETH Zurich in 2013 I thus decided to continue with an MSc in Biology at the University of Southern Denmark. For my MSc project I joined the Sound Communication and Behavior group at SDU. My thesis was supervised by Dr. Magnus Wahlberg and described the influence of anthropogenic noise on the communication range of harbor seal (Phoca vitulina) vocalizations. Following my MSc I started a $\mathrm{PhD}$ at Wageningen University, The Netherlands, to study the role of male bird song in intraand intersexual selection in the great tit (Parus major) under the supervision of Prof. Marc Naguib and Prof. Kees van Oers. During my PhD I also contributed to an ongoing collaboration project with Dr. Andy Young at Exeter University to study the function of male solo song in the white-browed sparrow weaver (Plocepasser mahali), a cooperative breeder. I have recently started working on the role of early life experience and genetics in song learning in pied flycatchers (Ficedula hypoleuca) as a postdoctoral research assistant at Stockholm University with Dr. David Wheatcroft. 


\section{WIAS Training and Education Statement}

The basic package

WIAS Introduction Day 2016

WGS course: Research Integrity \& Ethics and Animal Science 2016

ECTS total 2

Disciplinary competences

Laboratory Animal Science course (KNAW) 2016

(Introduction to Laboratory Animal Science \& species-specific bird course)

Basic Statistics using R (PE\&RC) 2016

WIAS literature review $\quad 2017$

$\begin{array}{ll}\text { Animal movement analysis (IBED) } & 2017\end{array}$

Mixed Linear Models (PE\&RC) 2017

$\begin{array}{ll}\text { ECTS total } & 13\end{array}$

Professional competences

Project and Time Management 2017

Communication with the Media and General Public 2017

Effective Behavior in your professional surroundings $\quad 2017$

WEES committee (Wageningen Ecology and Evolution seminar series): $\quad 2017$ - 2018

secretary \& organization of 3 seminars

Scientific Artwork: Vector graphics and images 2019

ECTS total

Presentation skills

Poster presentation: annual meeting of the Netherlands Society 2017

for Behavioral Biology, Soesterberg, NL

Poster presentation: Goettinger Freilandtage, Goettingen, DE

2017

Oral presentation: 27th International Ornithological Conference, Vancouver, CA 2018

Oral presentation: 56th annual conference of the Animal Behavior Society, 2019

Chicago, Illinois, USA

Oral presentation: summer meeting of the Association for the Study

2019

of Animal Behavior, Konstanz, DE 
Teaching competences

Msc major thesis project supervisions: 7

$2016-2019$

Msc minor thesis project supervisions: 1

2017

Bsc thesis project supervisions: 3

$2016-2018$

Internship project supervisions: 3

$2016-2018$

Assisting in practicals and excursions:

ABG-50806 (2017), BHE-20303 (2018-2019), NEM-21306 (2016-2018)

ECTS total 
The research described in this thesis was financially supported by the open competition grant number 824.15.012 to MN funded by the Dutch Research Council (NWO).

\section{Printed by}

proefschriftmaken.nl

\section{Cover illustration by}

Annika-Corinna Toth (ARTbyAnnikaToth) 


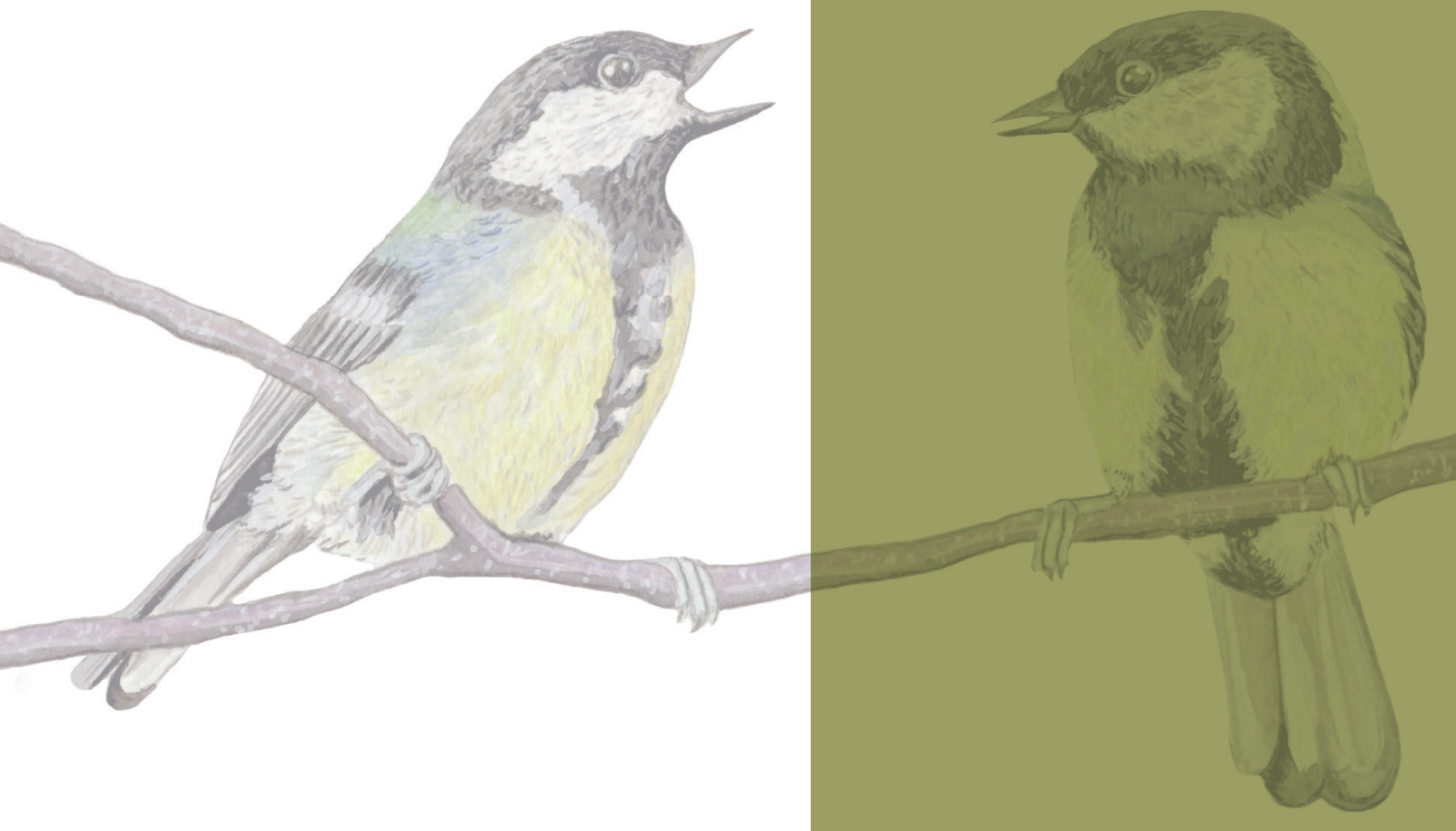

